Florida International University FIU Digital Commons

\title{
A Search for an Exotic Meson in the Gamma P to Delta-plus-plus Pi-minus Eta Reaction
}

Diane M. Schott

Florida International University, dscho001@fiu.edu

DOI: $10.25148 /$ etd.FI12071114

Follow this and additional works at: https://digitalcommons.fiu.edu/etd

\section{Recommended Citation}

Schott, Diane M., "A Search for an Exotic Meson in the Gamma P to Delta-plus-plus Pi-minus Eta Reaction" (2012). FIU Electronic Theses and Dissertations. 662.

https://digitalcommons.fiu.edu/etd/662

This work is brought to you for free and open access by the University Graduate School at FIU Digital Commons. It has been accepted for inclusion in FIU Electronic Theses and Dissertations by an authorized administrator of FIU Digital Commons. For more information, please contact dcc@fiu.edu. 


\section{FLORIDA INTERNATIONAL UNIVERSITY \\ Miami, Florida}

\section{A SEARCH FOR AN EXOTIC MESON IN THE $\gamma+P \rightarrow \Delta^{++}+\pi^{-}+\eta$ REACTION}

A dissertation submitted in partial fulfillment of the

requirements for the degree of

DOCTOR OF PHILOSOPHY

in

PHYSICS

by

Diane Schott 
To: Dean Kenneth G. Furton

College of Arts and Sciences

This dissertation, written by Diane Schott, and entitled A Search for an Exotic Meson in the $\gamma+P \rightarrow \Delta^{++}+\pi^{-}+\eta$ Reaction, having been approved in respect to style and intellectual content, is referred to you for judgment.

We have read this dissertation and recommend that it be approved.

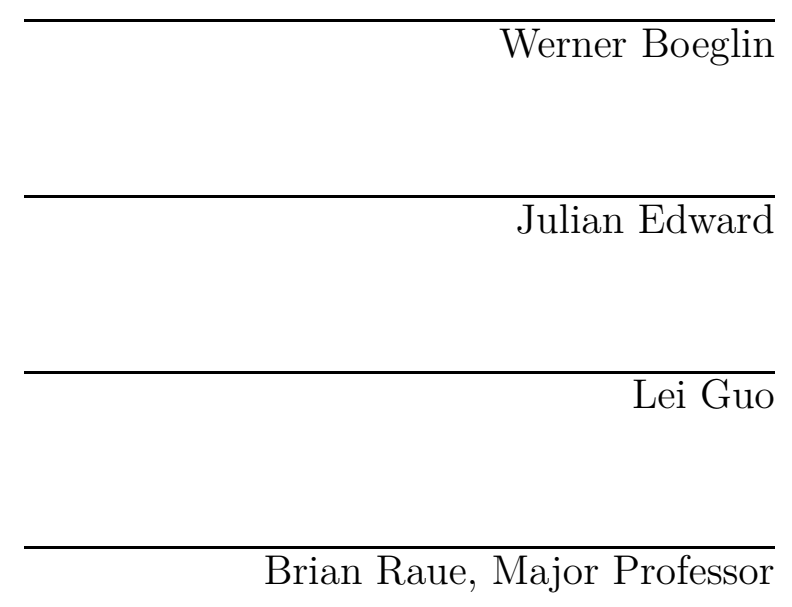

Date of Defense: June 22, 2012

The dissertation of Diane Schott is approved.

Dean Kenneth G. Furton

College of Arts and Sciences

Dean Lakshmi N. Reddi

University Graduate School

Florida International University, 2012 


\section{ACKNOWLEDGMENTS}

I would like to thank my advisor Dr. Brian Raue for his continuous support throughout my graduate career. I would also like to thank my committee members, Dr. Werner Boeglin, Dr. Julian Edward, and Dr. Lei Guo, for their time and effort reviewing my dissertation. Thanks also goes out to the core members of the g12 run group at Jefferson Lab. These people made the experiment that this dissertation is

based on successful. In addition, I would like to thank Dr. Dennis Weygand and Dr.

Carlos Salgado for taking interest in my work and for the valuable time spent discussing the analysis. I would also like to thank Dr. Paul Eugenio for his guidance and for helping me get started on the experiment. This thesis would not have been possible without the g12 graduate students and postdocs, so I owe my deepest gratitude to Johann Goetz, Craig Bookwalter, Mike Paolone, and Mukesh Saini. I would like to thank Johann Goetz for very patiently reviewing my dissertation and keeping me motivated with regular coffee breaks. I am grateful for the many times he helped influence me as a programmer. I would like to thank Mike Paolone for being a supportive force since we first met while I spent my first summer at Jefferson Lab in 2004. We've both come a long way since I was a summer intern in Hall A and Mike was a graduate student in Hall B. I would like to thank Craig Bookwalter for inspiring and motivating me to finish. I will be forever grateful for [him] helping me clear my mind with rock climbing. Finally, I would like to thank my family for supporting me over the years and for being extremely patient. 


ABSTRACT OF THE DISSERTATION
A SEARCH FOR AN EXOTIC MESON IN THE $\gamma+P \rightarrow \Delta^{++}+\pi^{-}+\eta$
REACTION
by
Diane Schott
Florida International University, 2012
Miami, Florida
Professor Brian Raue, Major Professor

A Partial Waves Analysis (PWA) of $\gamma p \rightarrow \Delta^{++} X \rightarrow p \pi^{+} \pi^{-}(\eta)$ data taken with the CLAS detector at Jefferson Lab is presented in this work. This reaction is of interest because the $\Delta^{++}$restricts the isospin of the possible $X$ states, leaving the PWA with a smaller combination of partial waves, making it ideal to look for exotic mesons. It was proposed by Isgur and Paton [1] that photoproduction is a plausible source for the $J^{p c}=1^{-+}$state through flux tube excitation. The $\pi_{1}(1400)$ is such a state that has been produced with the use of hadron production but it has yet to be seen in photoproduction. A mass independent amplitude analysis of this channel was performed, followed by a mass dependent fit to extract the resonance parameters. The procedure used an event-based maximum likelihood method to maintain all correlations in the kinematics. The intensity and phase motion is mapped out for the contributing signals without requiring assumptions about the underlying processes. The strength of the PWA is in the analysis of the phase motion, which for resonance behavior is well defined. In the data presented, the $\eta \pi^{-}$invariant mass spectrum shows contributions from the $a_{0}(980)$ and $a_{2}(1320)$ partial waves. No $\pi_{1}$ was observed under a clear $a_{2}$ signal after the angular distributions of the decay products were analyzed using an amplitude analysis. In addition, this dissertation discusses trends in the data, along with the implemented techniques. 


\section{TABLE OF CONTENTS}

CHAPTER

PAGE

1. INTRODUCTION AND HISTORICAL REVIEW . . . . . . . . . . . 1

1.1 Standard Model . . . . . . . . . . . . . . . . . . . . . 1

1.2 Quantum Chromo-Dynamics and Confinement . . . . . . . . . . . 3

1.3 Constituent Quark Model . . . . . . . . . . . . . . . . . . . . 4

1.4 Symmetries and Groups $\ldots \ldots \ldots \ldots \ldots \ldots$

1.4 .1 Isospin . . . . . . . . . . . . . . . . . . . . 5

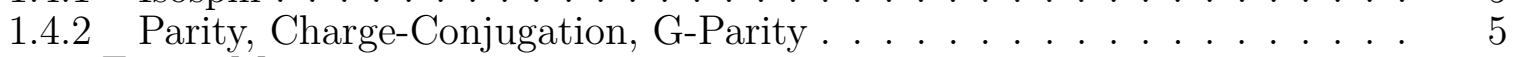

1.5 Exotic Mesons . . . . . . . . . . . . . . . . . . . . 6

1.5.1 Previous Observations and Conclusions . . . . . . . . . . . . . 7

1.6 Photoproduction of Exotic Mesons _ . . . . . . . . . . . . . . 10

1.7 This Experiment . . . . . . . . . . . . . . . . . . . . . . 12

2. EXPERIMENTAL SETUP . . . . . . . . . . . . . . . . . 13

2.1 The Continuous Electron Beam Accelerator . . . . . . . . . . 15

2.2 Hall B Photon Beam and Tagger . . . . . . . . . . . . . . . . . . 18

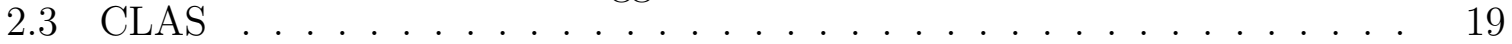

2.4 Hall B Data Readout . . . . . . . . . . . . . . . . . . . . . 26

2.5 Detector Calibration and Particle Identification . . . . . . . . . . 26

2.5 .1 Tagger Calibrations . . . . . . . . . . . . . . . . . 27

2.5 .2 Start Counter and Time-Of-Flight $\ldots \ldots \ldots \ldots \ldots . \ldots . \ldots 27$

2.5.3 Drift Chambers Calibration and Alignment . . . . . . . . . . . . 29

2.6 Data Reconstruction . . . . . . . . . . . . . . . . . . 33

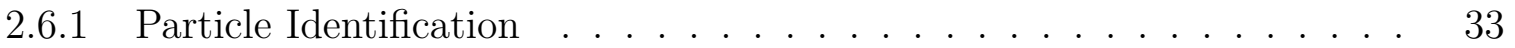

3. DATA ANALYSIS . . . . . . . . . . . . . . . . 36

3.1 Event Vertex and Beam Energy Cut . . . . . . . . . . . 36

3.2 Timing Cut . . . . . . . . . . . . . . . . . . . . . . . . 39

3.3 Energy Loss Corrections . . . . . . . . . . . . . . . . . . . . . . . 41

3.4 Missing Momentum Cuts . . . . . . . . . . . . . . . . . . . . . . 44

3.5 Additional Missing Mass and $\gamma \gamma$ Invariant Mass Cuts . . . . . . . . . 47

3.6 Kinematic Fitting . . . . . . . . . . . . . . . . . . . 50

3.7 Final Event Selection . . . . . . . . . . . . . . . . . . . . . 54

$3.7 .1 \Delta^{++} \rightarrow p \pi^{+}$Selection $\ldots \ldots \ldots \ldots \ldots \ldots \ldots \ldots \ldots$

$3.7 .2 \quad \eta \rightarrow \gamma \gamma$ Selection . . . . . . . . . . . . . . . 55

3.7 .3 Momentum Transfer Selection . . . . . . . . . . . . . . . . . . 57

3.7.4 Possible Interfering Processes to $\pi_{1}(1400)$ Production . . . . . . . . . . 61

4. PARTIAL WAVE ANALYSIS . . . . . . . . . . . . . . 66

4.1 Decay Amplitudes . . . . . . . . . . . . . . . . . . . . . . 68

4.2 Production Amplitude and Intensity _ . . . . . . . . . . . . 72

4.3 Maximum Likelihood Method . . . . . . . . . . . . . . . . . . . 74

4.4 Partial Wave Fitting Procedure . . . . . . . . . . . . . . . . 76

4.5 Experimental Acceptance . . . . . . . . . . . . . . . . . . . 77

4.6 Results. . . . . . . . . . . . . . . . . . . . 80

4.6 .1 Wave Set . . . . . . . . . . . . . . . . 81

4.6.2 PWA: Mass-Independent Fit . . . . . . . . . . . . . . . . . . . . . 82

4.6 .3 Mass-Dependent Fit . . . . . . . . . . . . . . . . . . . . . 87

4.7 Upper Limit on the $\pi_{1}(1400) \quad \ldots \ldots \ldots$ 
5. DISCUSSIONS AND CONCLUSIONS _ . . . . . . . . . . . 101

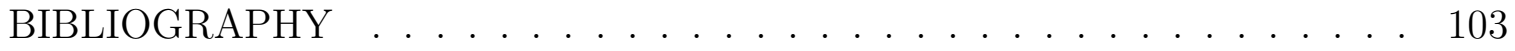

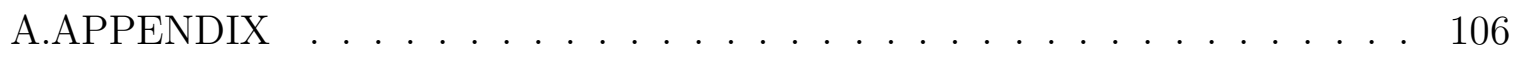

VITA . . . . . . . . . . . . . . . . . . . . 117 


\section{LIST OF TABLES}

TABLE

PAGE

1.1 Standard Model Fermions and Force Propagators. . . . . . . . . . . . . 3

1.2 A partial list of the first set of states allowed by $q \bar{q}$ and $q \bar{q} g$. . . . . . . 7

1.3 Summary of published results for $1^{-+}$states. . . . . . . . . . . . . 10

3.1 Summary of data cuts used. . . . . . . . . . . . . . . 65

4.1 Summary of the partial wave states and corresponding meson. . . . . . . 74

4.2 Summary of the partial wave states used in the final fit. . . . . . . . . . 82

4.3 Summary of the mass-dependent fits, all masses and widths are in units of $\mathrm{GeV}$. . . . . . . . . . . . . . . . . . . . . . . . . . 90 


\section{LIST OF FIGURES}

FIGURE

PAGE

1.1 The relations for spin $(S)$, parity $(P)$, angular momentum $(L)$, chargeconjugation $(C)$, and total angular momentum $(J)$ within a meson. . . 2

1.2 The octet of mesons. . . . . . . . . . . . . . . . . . . . . . . . 2

1.3 Results from the analysis of E852. . . . . . . . . . . . . 8

1.4 The results from the CBAR for the reaction $\bar{p} d \rightarrow p \pi^{-} \pi^{0} \eta$. . . . . . . . 9

1.5 Quark spin alignment in photoproduction and pion production. . . . . . 11

1.6 Feynman diagram of $\gamma p \rightarrow \Delta^{++} X \ldots \ldots \ldots$. . . . . . . . . . 12

2.1 Hall B layout. . . . . . . . . . . . . . . . . . . . . . 14

2.2 An illustration of electrons accelerated through a RF cavity. . . . . . . . 16

2.3 Layout of the CEBAF accelerator. . . . . . . . . . . . . . . 17

2.4 Side view of the tagger. . . . . . . . . . . . . . . . 19

2.5 Side view of CLAS. . . . . . . . . . . . . . . . . . 21

2.6 Diagram of the start counter. . . . . . . . . . . . . . . . 22

2.7 Diagram of the regions and super layers of the DC. . . . . . . . . . . 23

2.8 View parallel to the $\mathrm{DC}$ wires. . . . . . . . . . . . . . . 24

2.9 Diagram of one sector of the TOF paddles. . . . . . . . . . . . . . 24

2.10 Electromagnetic Calorimeter sectional view. . . . . . . . . . . . . 25

2.11 Run by run plot of the sigma from the double gaussian fit on the residual fit of the DC. Sigma is the physical resolution of the chamber. . . . . 31

2.12 Run by run plot of the mean from the double gaussian fit on the residual fit of the $\mathrm{DC} \ldots \ldots . \ldots 31$

2.13 Alignment of sector 4, before and after the final DC alignment. . . . . . 32

2.14 Flow chart of reconstruction process for CLAS. . . . . . . . . . . . . 34

3.1 The $x, y, z$ coordinate plots of the $p \pi^{+}$intersection. . . . . . . . . . . 37

3.2 The photon beam energy. . . . . . . . . . . . . . . . . 38

3.3 Photons in timing bucket. . . . . . . . . . . . . . . . . 39 
3.4 Plots of the difference in start counter vertex time to RF-corrected tagger vertex time and difference between the $\mathrm{RF}$-corrected tagger vertex time and the vertex time calculated from the assumed mass value. . . . .

3.5 The $\beta$ of the track compared to the momentum before and after the timing and particle ID cuts. . . . . . . . . . . . . . . 41

3.6 The energy loss corrections applied using ELOSS. . . . . . . . . . . . . . 43

3.7 Plot of the missing transverse momentum off the $p \pi^{+} \pi^{-} \ldots \ldots . . . .45$

3.8 Plot of the missing mass squared off of the $p \pi^{+} \pi^{-} \ldots \ldots$. . . . . . . 46

3.9 Missing mass squared off of $p \pi^{+} \pi^{-} \gamma \gamma \ldots \ldots$. . . . . . . . . . . 48

3.10 The calculated difference between $\theta$ of the $M M\left(p \pi^{+} \pi^{-}\right)$and $\gamma \gamma$. . . 48

3.11 The calculated difference between $\phi$ of the $M M\left(p \pi^{+} \pi^{-}\right)$and $\gamma \gamma$ detected. 49

3.12 Missing mass squared off of $p \pi^{+} \pi^{-}$with the $\gamma \gamma$ detected. . . . . . . . . 49

3.13 Kinematic fit results of $p \pi^{+} \pi^{-}\left(\pi^{0}\right)$ where one and two $\gamma^{\prime}$ 's were detected. 51

3.14 Missing mass squared off of $p \pi^{+} \pi^{-} \ldots \ldots \ldots \ldots$

3.15 The final results of the kinematic fit of $p \pi^{+} \pi^{-}(\eta) \ldots \ldots . . \ldots 54$

3.16 Invariant mass of $p \pi^{+}$after kinematic fit for $p \pi^{+} \pi^{-}(\eta)$ and the CL cut. . 56

3.17 Invariant mass of $p \pi^{-}$after the $\Delta^{++}$invariant mass selection. . . . . . . 56

3.18 Plot of the invariant mass of $\gamma \gamma$ after the CL cut. . . . . . . . . . 57

3.19 Invariant mass of $\eta \pi^{-} \ldots \ldots \ldots \ldots$. . . . . . . . . . . . . . . . .

3.20 Invariant mass of $\eta \pi^{-}$, with mass regions highlighted. . . . . . . . . . . 59

3.21 The momentum transfer, $t$, of the events under the $a_{2}(1320)$ peak. . . . 60

3.22 Invariant mass of $\eta \pi^{-}$after $t$ cut. . . . . . . . . . . . . . . 61

3.23 Invariant mass distribution of $\eta \pi^{+}$in the low $|t|$ range. . . . . . . . . . . 62

3.24 Invariant mass distribution of $\eta p$ in the low $|t|$ range. . . . . . . . . . . 63

3.25 Invariant mass distribution of $\pi^{+} \pi^{-} \ldots \ldots \ldots$. . . . . . . . . . . 63

3.26 Invariant mass distribution of $\pi^{+} \pi^{-}$in the low $|t|$ range. . . . . . . . . 64

3.27 Invariant mass distribution of $\eta \pi^{+} \pi^{-}$in the low $|t|$ range. . . . . . . . 64 
3.28 Invariant mass distribution of $\eta p \pi^{+}$in the low $|t|$ range. . . . . . . . . . 65

4.1 Feynman diagram of $\gamma p \rightarrow \Delta^{++} X$ for PWA . . . . . . . . . . 67

4.2 Decay of $X$ to $\eta$ and $\pi^{-}$in lab frame. . . . . . . . . . . . 68

4.3 Diagram of the Gottfried-Jackson angles. . . . . . . . . . . . . . . 69

4.4 Decay of $X$ to $\eta$ and $\pi^{-}$in rest frame of the resonance, with the $\hat{z}$ direction defined as the beam direction. . . . . . . . . . . . . 71

4.5 Decay of $X$ to $\eta$ and $\pi^{-}$in rest frame of the resonance, with the $\hat{z}$ direction defined as the meson momentum direction. . . . . . . . . . . 71

4.6 Invariant mass of $\eta \pi^{-}$for the PWA. . . . . . . . . . . . . 75

$4.7 t$ distribution of the simulation. . . . . . . . . . . . . . 79

$4.8 t$ distribution of the data after sideband subtraction. . . . . . . . . . 79

4.9 Acceptance of $p \pi^{+} \pi^{-} \gamma \gamma$ over the invariant mass of $\eta \pi^{-} . \ldots . . . . .80$

4.10 Intensity distributions of final wave set. Using a fit with the partial waves $2^{+} 1,1^{-} 1$, and $0^{+} 0^{-} \ldots \ldots \ldots \ldots$

4.11 Intensity distributions of final wave set. Using a fit with the partial waves $2^{+} 1,1^{-} 1$, and $0^{+} 0^{-} \ldots \ldots \ldots \ldots$

4.12 Phase differences of final wave set. Using a fit with the partial waves $2^{+} 1$, $1^{-} 1$, and $0^{+} 0^{-} \ldots \ldots \ldots \ldots \ldots \ldots$

4.13 The GJ angles for the data (points) used in the PWA fit overlaid with the predicted distribution from the PWA solution (histogram). . . .

4.14 Invariant masses of $p \pi^{+} \eta$ and $p \eta$ of the data in red, with the invariant masses predicted by the PWA solution in blue. . . . . . . . . . . .

4.15 Invariant masses of $\pi^{+} \pi^{-} \eta$ and $\pi^{+} \eta$ of the data in red, with the invariant masses predicted by the PWA solution in blue. . . . . . . . . . .

4.16 Fit 1: Intensity mass dependent fit for $a_{2} \ldots \ldots$. . . . . . . . . 94

4.17 Calculated BW phase differences. . . . . . . . . . . . . . . . 95

4.18 Fit 2: Coupled phase motion and intensity mass dependent fit for $a_{2}$. . 96

4.19 Fit 3: Coupled phase motion and intensity mass dependent fit for $a_{2}$ and $\pi_{1}$, for positive reflectivity. . . . . . . . . . . . .

4.20 Fit 4: Coupled phase motion and intensity mass dependent fit for $a_{2}$ and $\pi_{1}$, for positive reflectivity. . . . . . . . . . . 98 
4.21 Coupled Phase Motion and Intensity Mass Dependent Fit for $a_{2}$ and $\pi_{1}$, using a fixed $\pi_{1}$ mass and width from E852 . . . . . . . 100

A.1 Intensity distributions for the PWA using waves $2^{+} 0^{-}, 2^{+} 1,1^{-} 0^{-}, 1^{-} 1$ and $0^{+} 0^{-} \ldots \ldots \ldots \ldots \ldots \ldots \ldots \ldots \ldots \ldots \ldots \ldots \ldots$

A.2 Intensity distributions for the PWA using waves $2^{+} 0^{-}, 2^{+} 1,1^{-} 0^{-}, 1^{-} 1$ and $0^{+} 0^{-} \ldots \ldots \ldots \ldots \ldots \ldots \ldots$

A.3 Intensity distributions for the PWA using waves $2^{+} 0^{-}, 2^{+} 1,1^{-} 1$ and $0^{+} 0^{-} .110$

A.4 Intensity distributions for the PWA using waves $2^{+} 0^{-}, 2^{+} 1,1^{-} 1$ and $0^{+} 0^{-} .111$ 


\section{CHAPTER 1 INTRODUCTION AND HISTORICAL REVIEW}

Exotic states are states not described solely by the constituent quark model, CQM, in which the meson is described only by the valence quarks. The first discovered [2] resonant mesonic state was the $\rho$ in the 1950s. The finding lit the way to finding new states of matter. Since then, exotic mesons have been found in a number of hadron-production experiments. The lightest of these states is the $\pi_{1}(1400)$ decaying to $\eta \pi^{-}$, initially observed by E852 collaboration at Brookhaven [3] in the reaction $\pi^{-} p \rightarrow \eta \pi^{-} p$. With limited photoproduction data, there has not been a conclusive analysis of the photoproduced $\eta \pi^{-}$because of a lack of statistics. Photoproduction is predicted to favor production of the exotic state resulting in the increase of the ratio of $\pi_{1}$ to $a_{2}$ mesons [4].

\subsection{Standard Model}

In the Standard Model, all matter is made up of leptons and quarks that interact through the exchange of gauge bosons. Table 1.1 shows the matter and interaction propagators for the standard model. Leptons interact electromagnetically using photons as propagators. $99.9 \%$ of the universe's known mass is made up of hadronic particles. From the Standard Model, a nonet of mesons shown in Fig. 1.2 is formed from combinations of up $(u)$, down $(d)$, and strange $(s)$ quarks. These particles have been detected experimentally [2].

The force between quarks can be described as the exchange of particles called gluons. The gluon is a neutral and massless vector particle with spin-parity $J^{P}=1^{-}$, where $J$ is the total angular momentum and defined as the sum of the orbital angular

momentum, $L$, and spin, $S$, as shown in Fig. 1.1. Gluons can interact with themselves, since they carry the color charge that they couple to, whereas photons do not couple 


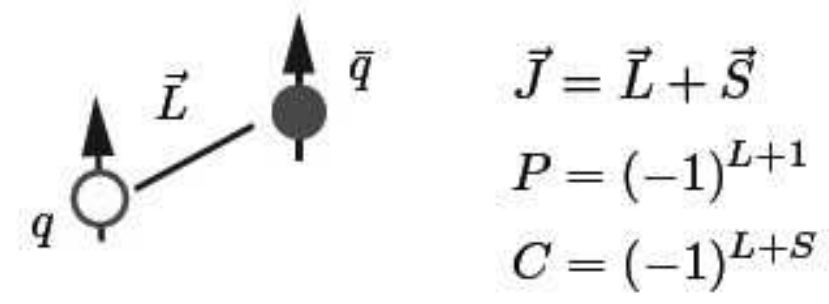

Figure 1.1: The relations for spin $(S)$, parity $(P)$, angular momentum $(L)$, chargeconjugation $(C)$, and total angular momentum $(J)$ within a meson. Where $S$ is the total spin of the individual quarks, $L$ is the angular momentum of the system. These are used to define $J$ as $|L-S| \leq \mathrm{J} \leq|L+S|$. Spin projection $(m)$ is defined as the projection in the $z$ direction of $s$ resulting in $m=-S,-S+1, \ldots 0, \ldots, S-1, S$.

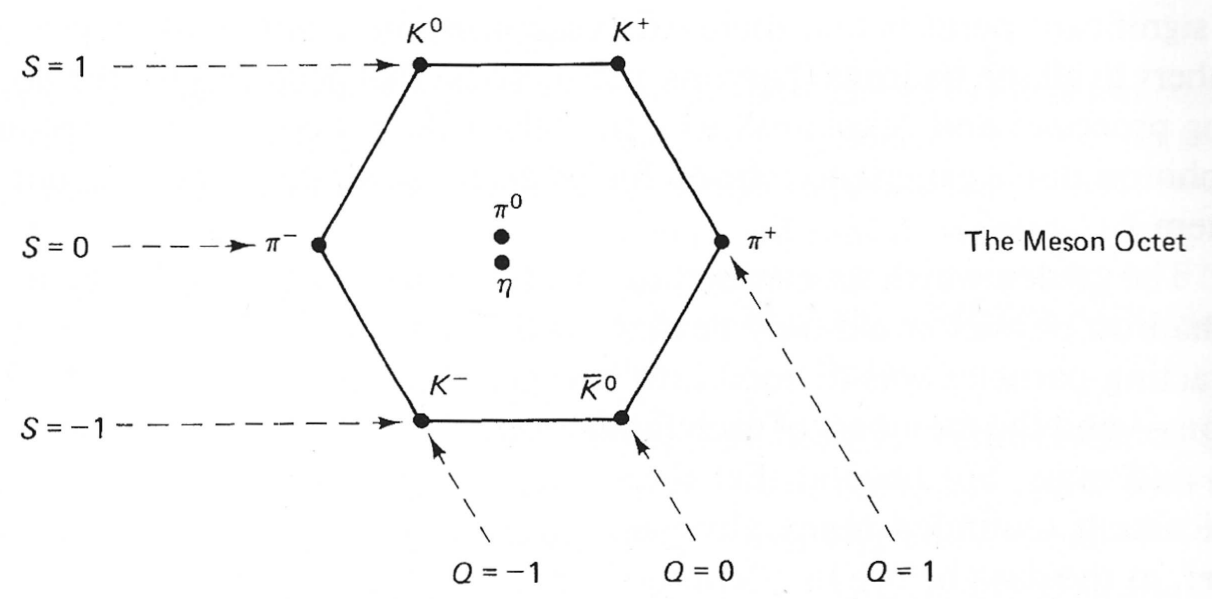

Figure 1.2: The nonet of mesons formed by the quark model and is the octet shown plus a singlet. $(\bar{u} d, \bar{d} u, \bar{u} u \pm \bar{d} d, \bar{s} s, \bar{u} s, \bar{d} s, \bar{s} u, \bar{s} d)[6]$.

with other photons since their electromagnetic charge is zero. A quark carries one of three colors (red, green, or blue) and it's antiquark carries the anti-color. For example, a pair of red and anti-blue quarks inside a meson are held together through the exchange of a blue anti-red gluon. 


\begin{tabular}{|c|c|c|c|}
\hline Fermions & & & \\
\hline & Generation 1 & Generation 2 & Generation 3 \\
\hline Quarks & up $(u)$ & Charm $(c)$ & Top $(t)$ \\
& Down $(d)$ & Strange $(s)$ & Bottom $(b)$ \\
\hline Leptons & Electron Neutrino & Muon Neutrino & Tau Neutrino \\
& $\left(\nu_{\phi}\right)$ & $\left(\nu_{\mu}\right)$ & $\left(\nu_{\tau}\right)$ \\
& Electron $(e)$ & Muon $(\mu)$ & Tau $(\tau)$ \\
\hline \hline Force Propagators & & & Strong Nuclear \\
\hline Force & Electromagnetic & Weak Nuclear & Gluons \\
\hline Propagator & Photon & Gauge Bosons & $(g)$ \\
& $(\gamma)$ & $\left(\mathrm{W}^{+}, \mathrm{W}^{-}\right.$, and Z) & \\
\hline
\end{tabular}

Table 1.1: Fermions are split into two categories, leptons and quarks [2]. Leptons are found in solitary ground states. Quarks, on the other hand, have never been found unbound and exist in states of two or more quarks. The mass of fermions increases with generation. Photons, gauge bosons and gluons propagate the basic forces of the universe; strong nuclear, weak nuclear, and electromagnetic forces.

\subsection{Quantum Chromo-Dynamics and Confinement}

For electromagnetic and weak forces, as two bodies are separated, the forces acting between them are expected to decrease as distance increases until the forces are so small it is as if the two bodies act independently of each other. The strong force acts between quarks and does not diminish in strength with an increasing distance resulting in quarks existing in pairs and triplets but never as isolated quarks. When a pair of quarks, consisting of a $d$ quark and $\bar{u}$ quark, are pulled apart, a new $q \bar{q}$ pair appears, resulting in two mesons in the final state. Quantum Chromo-Dynamics (QCD) is necessary to describe such processes.

QCD is a theory of the strong interaction that describes the interactions of quarks and gluons [7]. It is a gauge field theory describing the color $\mathrm{SU}(3)$ group. The two most significant consequences of QCD are confinement and asymptotic freedom. Confinement is a phenomenon where the force between quarks does not diminish as they are separated. As four-momentum transfer decreases, the quark-quark poten- 
tial becomes larger, in comparison, until the the potential reaches a pair production threshold and it is more favorable to have a spontaneous pair than to continue separating the quarks. It explains why quarks cannot be found isolated and have only been found bound to other quarks forming color singlets. Confinement has not been proven analytically but has been modeled in lattice QCD. Asymptotic freedom refers to quarks and gluons interacting weakly in very high energy reactions. Since the coupling constant of $\mathrm{QCD}, \alpha_{s}$, is a function of the four-momentum transfer $Q^{2}$, at short distances $Q^{2}$ is large and $\alpha_{s}$ is small, therefore the interaction can be treated with perturbation theory.

\subsection{Constituent Quark Model}

There are many models for explaining the hadron spectra in strong decays of hadrons. One model is the constituent quark model (CQM) which follows the work of GellMann and Zweig $[8,9]$. Initially only the up, down, and strange quarks were considered in the model, but eventually the charm, top, and bottom quarks were included. The model is built on a quasi-particle, where a many-body origin for the quark potential is assumed. For mesons this means the $q \bar{q}$ pairs are in bound states with the possibility for the pairs to be in excited states leading to a spectrum of masses for each $q \bar{q}$ pair combination. As the mass range decreases, there are states that are unexplained by the CQM.

The CQM describes hadrons using symmetries, or the invariance of the quark system after a transformation. Symmetries such as energy conservation and angular momentum conservation (for example) are used to determine the properties of the particles. When there is a break in the symmetry, it sheds light on other degrees of freedom of a given system. Generally, a broken symmetry exposes previously unknown 
symmetries and new properties are defined to describe the system. These properties are discussed in the next section.

\subsection{Symmetries and Groups}

\subsubsection{Isospin}

Isospin $(I)$ is defined as the interchangeability of the two lightest quarks, and results in a special organization of hadronic states corresponding to the $\mathrm{SU}(2)$ group [6]. Isospin has its origins in examining the proton (uuu) and neutron (uud), as they have almost the exact same mass and the nuclear force does not distinguish between the two. Each have $I=1 / 2$ with the projection of the isospin in the $z$ direction to be $I_{3}=+1 / 2$ and $-1 / 2$ for the proton and neutron, respectively. In the case of pions where $I=1, I_{3}$ is -1 for $\pi^{-}, 0$ for $\pi^{0}$, and 1 for $\pi^{+}$.

\subsubsection{Parity, Charge-Conjugation, G-Parity}

Parity $(P)$, charge conjugation $(C)$, and G-parity $(G)$ are discrete finite symmetry groups, each having two eigenvalue elements: \pm 1 . Parity is a mirroring in spatial coordinates about the origin resulting in the flip of the spatial coordinates' sign, and is conserved in strong and electromagnetic interactions but not for weak interactions. The parity of a meson is

$$
P=(-1)^{L+1}
$$

where $L$ is the orbital angular momentum between the $q$ and $\bar{q}$. The space inversion in the angular part of the $q \bar{q}$ wave function leads to the $(-1)^{L}$ factor. The additional -1 factor is due to the $q$ and $\bar{q}$ having opposite intrinsic parity.

Charge conjugation reverses the sign of the charge and magnetic moment of a particle, transforming the particle into the antiparticle. The charge conjugation eigen- 
value is

$$
C=(-1)^{L+S}
$$

where $S$ is the total spin of the $q \bar{q}$ pair. The $(-1)^{S+1}$ factor comes from $q \bar{q}$ composite spin states. A factor of -1 is from the interchanging of two fermions and the $(-1)^{L}$ is from the space inversion of the angular part of the wave function. Only neutral particles have $C$-parity eigenstates. The combination of parity and charge-conjugation $(C P)$ results in a reversal of the spacial coordinates and electromagnetic fields.

The charge conjugation followed by a 180 degree rotation around the axis of the second component of the isospin vector $\left(I_{2}\right)$ is $G$-parity. The eigenvalue is found by

$$
G=C(-1)^{I}
$$

where the $(-1)^{I}$ is from the rotation in isospin space. With the additional rotation, $G$-parity eigenvalues are found for both neutral and charged particles.

\subsection{Exotic Mesons}

QCD presents the possibility to have states not described by the constituent quark model (CQM). We call these states "exotic" mesons since they have quantum numbers not included in the quark model as described by

$$
J=L+S
$$

equation 1.1, and equation 1.2. States outside the quark model include glueballs $(g g)$, multi-quark states $(q \bar{q} q \bar{q})$, hybrid states $(q \bar{q} g)$ and meson-meson bound states. A leading model to describe these systems is the flux-tube model [1], which depends on the $q \bar{q}$ being held together by a cylindrical tube of color flux, where the binding energy is proportional to the tube length. With the gluon modeled as a physical object, the gluon is not limited to a ground state and quantum numbers not allowed 
in the constituent quark model are possible. Table 1.2 shows a sample of the states formed by $q \bar{q}$ and $q \bar{q} g$. The flux-tube model predicts a low mass exotic states of $J^{P C}=1^{-+}$with a mass of approximately $1.9 \mathrm{GeV}$. Exotic mesons have also been predicted using the bag model. In the bag model, quark and gluon fields are confined to a limited volume. This model predicts four nonets of exotic hybrids, with the lightest of $J^{P C}=1^{-+}$at $1.4 \mathrm{GeV}$.

\begin{tabular}{|c|c|c|c|}
\hline \multicolumn{4}{|c|}{ States } \\
\hline & $J$ & $P$ & $C$ \\
\hline$q \bar{q}$ & 0 & + (for s: odd) & + \\
\hline & & - (for s: even) & + \\
\hline & 1 & - & - \\
\hline & & + & - \\
\hline & 2 & + & + \\
\hline & & - & + \\
\hline$q g \bar{q}=q \bar{q}^{*} 1^{+-}$ & 0 & + & - \\
\hline & & - & - \\
\hline & 1 & - & + \\
\hline & & + & + \\
\hline & 2 & + & - \\
\hline & & - & - \\
\hline
\end{tabular}

Table 1.2: A partial list of the first set of states allowed by $q \bar{q}$ and $q \bar{q} g$. Where $g$ contributes $J^{p c}=1^{+-}$to the composite meson.

\subsubsection{Previous Observations and Conclusions}

Experiments at the Brookhaven National Laboratory (BNL) and the European Organization for Nuclear Research (CERN) have found evidence for a $J^{p c}=1^{-+}$exotic meson state by using partial wave analysis (PWA) technique as described in Chapter 4. At BNL, experiment E852 used two reactions to analyze $\eta \pi^{-}$and $\eta \pi^{0}$ resonances. First the reaction $\pi^{-} p \rightarrow \eta \pi^{-} p$ with a beam momentum of $18.3 \mathrm{GeV} / c$ and reconstructed a $\pi_{1}(1400)$ with $J^{p c}=1^{-+}$decaying to $\eta \pi^{-}[3,10]$. The PWA showed a 


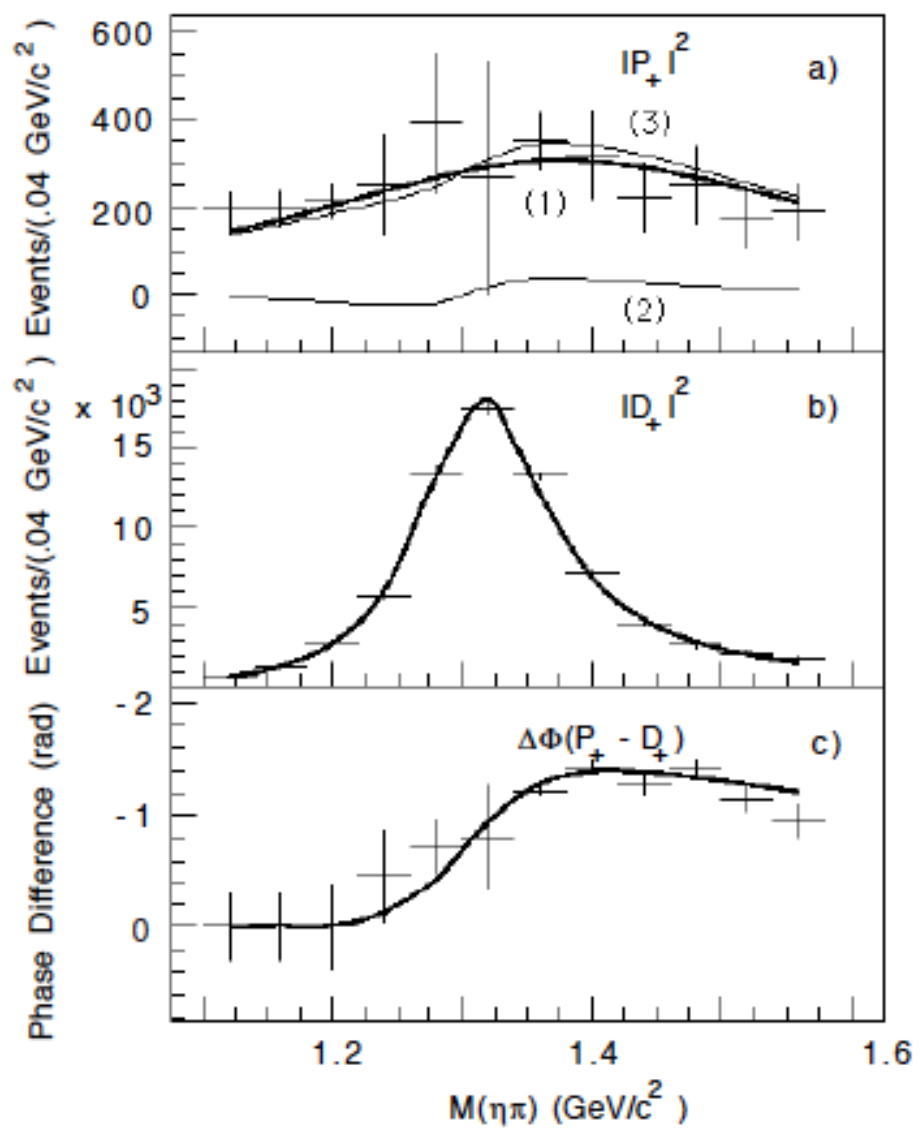

Figure 1.3: Results from the analysis of E852 [3]. Plot (a) shows the $P$ wave channel. This peak is poorly defined but in plot (c) the phase difference in the $P$ and $D$ wave shows interference at the mass of the $\pi_{1}(1400)$. Plot (b) shows the $D$ wave channel with a well defined peak at the mass of $1.317 \pm 0.003 \mathrm{GeV}$ and a width of $0.127 \pm$ $0.004 \mathrm{GeV}$.

clear $D_{+}{ }^{1}$ signal and interference between $D$ and $P$ waves, as shown in Fig. 1.3. The second reaction analyzed is the $\pi^{-} n \rightarrow \eta \pi^{0} p$ with the same beam momentum and had similar observations. No consistent set of amplitude parameters was found when the spin projection was included [11].

The Crystal Barrel Collaboration (CBAR) at CERN searched for a $J^{p c}=1^{-+}$ state in two ways. In the first reaction, $\bar{p} p \rightarrow \pi^{0} \pi^{0} \eta$ (isospin $I=0$, with a liquid

\footnotetext{
${ }^{1}$ Standard convention in physics is to denote various quantum states is to express $L=0$ as $S, L=1$ as $P$, and $L=2$ as $D$.
} 

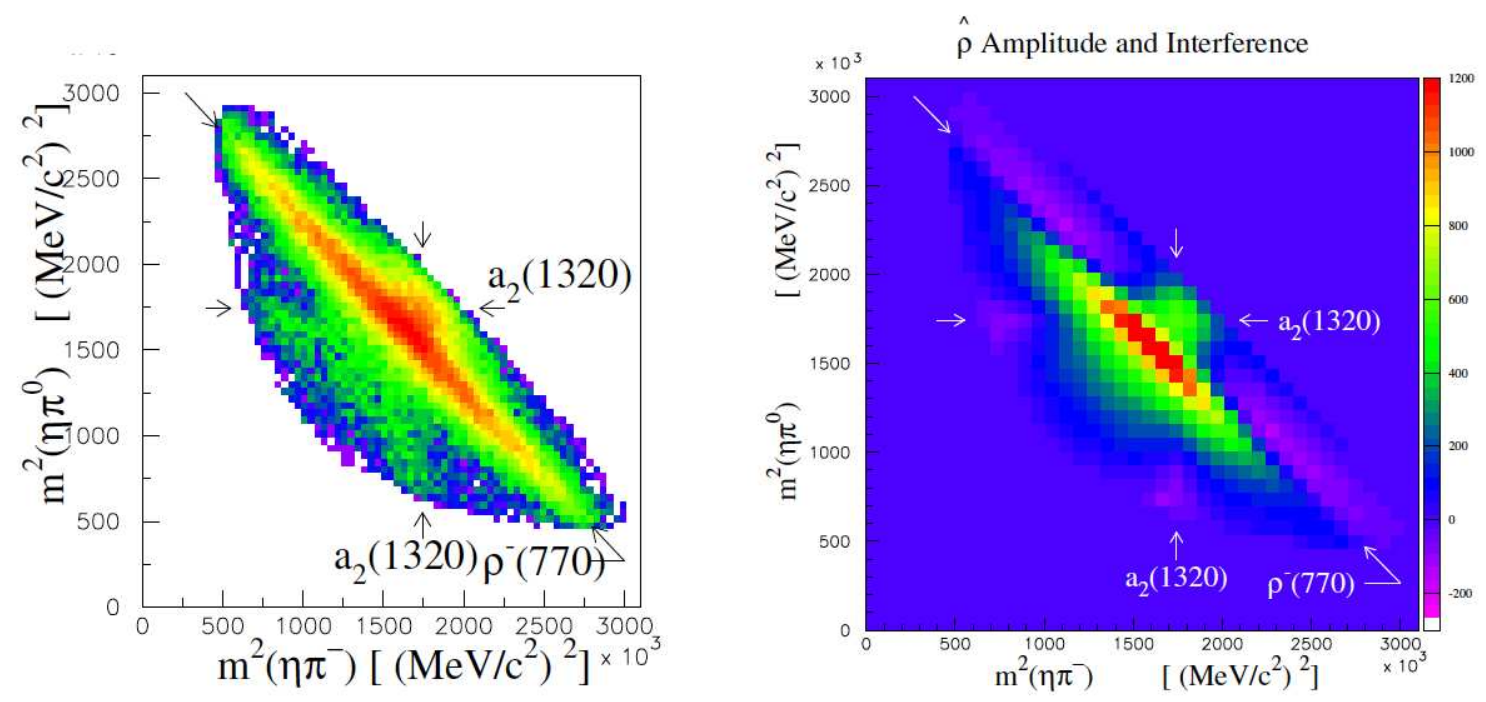

Figure 1.4: The results from the CBAR for the reaction $\bar{p} d \rightarrow p \pi^{-} \pi^{0} \eta$ [13]. The left plot is for the reaction $\bar{p} n \rightarrow \pi^{-} \pi^{0} \eta$, where the dominant band is the $\rho^{-}(770)$. Also in the plot are vertical and horizontal bands corresponding to the $a_{2}(1320)$. The right plot shows the contribution of the exotic $\pi_{1}(1400)$ and is important in explaining the data due to the $\pi_{1}$ state interfering with the $\rho$ and $a_{2}$

hydrogen target), the $\pi^{0} \eta$ consisted mainly of ${ }^{1} S_{0}$ (where the formalism used for the states is ${ }^{2 \mathrm{~S}+1} L_{\mathrm{J}}$ ) but had some ${ }^{3} P_{0}$ contributions [12]. This is attributed to $S$-wave annihilation. The mass and width of the $1^{-+}$state was poorly defined and is thought to be the result of the data being dominated by the ${ }^{1} S_{0}$ state, which would suppress $\pi_{1}$ production. In the second reaction $\bar{p} n \rightarrow \pi^{-} \pi^{0} \eta(I=1$, with a liquid deuterium target), the states were dominated by ${ }^{3} S_{1}$ and ${ }^{3} P_{1}$ [13]. Studying $\bar{p}$ on deuterium, the $\pi_{1}$ was well defined (see Fig. 1.4) and showed that the $\pi_{1}$ favors being produced by triplet states for $\bar{p} N$ reactions [11]. Table 1.3 summarizes the current findings for the $\pi_{1}(1400)$. 


\begin{tabular}{|l|l|l|l|l|}
\hline \multicolumn{5}{|c|}{ Past Results } \\
\hline Exp. Name & Beam/target & Decay Channel & Mass $\left(\mathrm{MeV} / \mathrm{c}^{2}\right)$ & Width $\left(\mathrm{MeV} / \mathrm{c}^{2}\right)$ \\
\hline & & $\rho \pi$ & $1593 \pm 8_{-47}^{+29}$ & $168 \pm 20_{-12}^{+150}$ \\
E852 & $\pi / p$ & $\eta \pi$ & $1370 \pm 16_{-30}^{+50}$ & $385 \pm 40_{-105}^{+65}$ \\
at BNL & & $\eta^{\prime} \pi$ & $1597 \pm 10_{-10}^{+45}$ & $360 \pm 40 \pm 50$ \\
\hline CBAR & $\bar{p} d$ & $\eta \pi$ & $1400 \pm 28$ & $310_{-58}^{+71}$ \\
at CERN & $p \bar{p}$ & $\eta \pi$ & $1360 \pm 25$ & $220 \pm 90$ \\
\hline
\end{tabular}

Table 1.3: Summary of published results for $1^{-+}$states. The first error is statistical, the second represents the systematic uncertainties $[11,3]$.

\subsection{Photoproduction of Exotic Mesons}

Historically, most studies for exotic mesons have used hadron beams and there are very limited data on photoproduction of light-quark mesons. In pion production experiments, the valence quarks are anti-aligned but in photoproduction the vector meson has aligned quarks to preserve the spin of the photon, as illustrated in Fig. 1.5. The result is, in the pion case, the quarks will have to realign and excite the flux tube but the photoproduction case will only need to excite the flux tube. This has led to the hypothesis that photoproduction will be a better source to study $J^{P C}=1^{-+}$exotic resonances, in terms of a large cross section. It has been suggested by Szczepaniak [4] that exotic mesons will be produced with intensities ten times that seen using hadron beams relative to the $a_{2}$. This prediction is based on the use of Regge propagator in the amplitude of the resonance. The ratio between the $\pi_{1}$ and $a_{2}$ is then expressed proportional to the ratio of the coupling constants between the resonance, beam, and exchange particle. This makes an assumption on the possible range of the coupling constant of the $\pi_{1}$. Of the light meson photoproduction experiments that have been done, the statistics have been low, making a partial wave analysis (PWA) difficult and inconclusive $[14,15]$. Previously, using CLAS at Jefferson Lab, the experiment g6c investigated $\gamma p \rightarrow \Delta^{++} \eta \pi^{-}$. After event selection and background reduction, there 

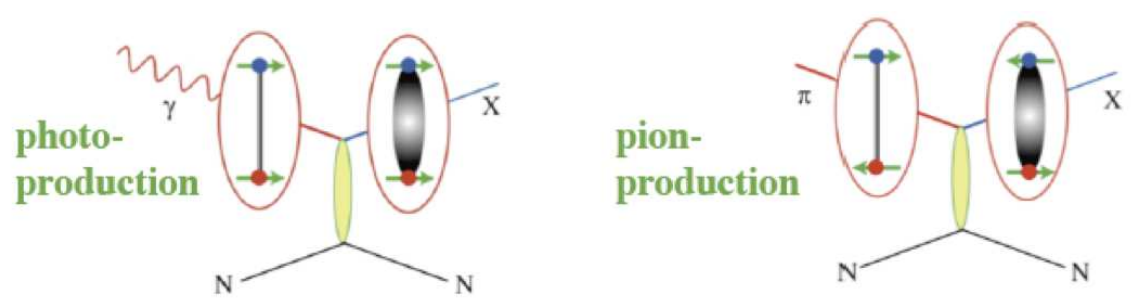

Figure 1.5: Quark spin alignment in photoproduction and pion production.

were not enough events for a PWA to separate out the background and determine the contributing signal waves [15].

The $\gamma p \rightarrow p \pi^{+} \eta \pi^{-}$reaction does not conserve isospin because of the photon. A photon has an isospin of either 0 or 1 . One approach is to use Vector Meson Dominance (VMD). VMD treats the photon as sum of vector-meson $(\rho, \omega$, and $\phi)$ contributions. Where the photoproduction amplitude becomes

$$
A(\gamma p)=\frac{1}{g_{\rho}} A(\rho p)+\frac{1}{g_{\omega}} A(\omega p)+\frac{1}{g_{\phi}} A(\phi p) .
$$

The $\phi$ contribution is minimal and can be neglected because the $\phi$ is made up of the heavier $s \bar{s}$ pair and there are no strange quarks in this reaction. This leaves the $\rho$ and $\omega$ as possible contributions. Comparing the coupling constants, $g_{\rho}$ and $g_{\omega}$, the $\rho$ is favored by $9: 1$ [16].

The exchanged particles are expected to be $\pi$ and $\rho$. The $\pi$ exchange is due to the photon interacting as a $\rho^{0}$ and the possible $\rho$ exchange is from the photon coupling as a $\omega^{0}$. The $\pi$ exchange contributes to the $a_{2}$ and $\pi_{1}$ production, while the $a_{0}$ is produced from a $\rho$ exchange. The next heavier exchange particle is the $a_{2}$, the expected contribution is calculated to be suppressed by a factor of $\sqrt{2}$ when compared to the $\rho$ exchange, as mentioned in Ref. [17]. 
$\eta$

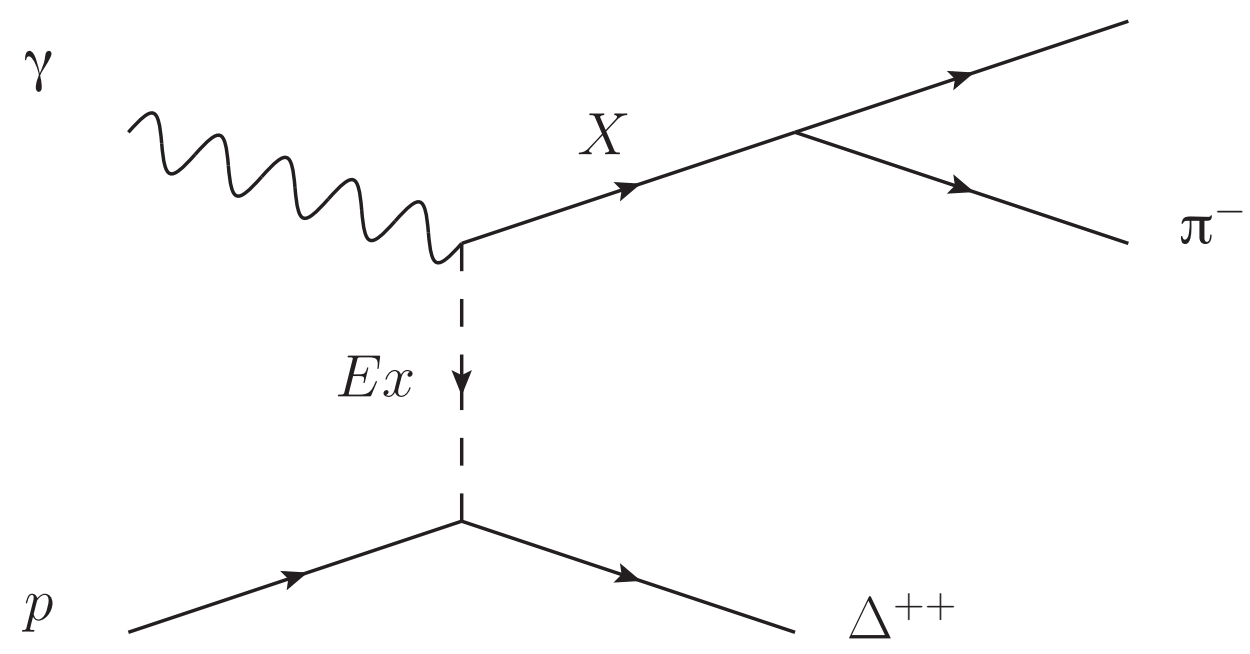

Figure 1.6: Feynman diagram of $\gamma p \rightarrow \Delta^{++} X$.

\subsection{This Experiment}

The g12 experiment was designed to examine meson spectroscopy with a low momentum transfer for the possibility of finding exotic mesons. The reaction $\gamma p \rightarrow \Delta^{++} X$, Fig. 1.6, is an interesting channel because, by including the $\Delta^{++}$, the exchange particle is restricted to be a $\pi$ or $\rho$ resulting in a resonance with spin projection of $|m|=1$ or 0 and rules out higher order resonance states $(|m|>1)$. The threshold energy for a particle with the mass of an $a_{2}(1320)$ or $\pi_{1}(1400)$ is around $3 \mathrm{GeV}$. The beam energy for the analysis is set at $E_{\gamma}>4.5 \mathrm{GeV}$ to match the trigger configuration. The final state of this channel is $p \pi^{+} \pi^{-} \eta$, requiring the identification of three charged particles, making this an ideal experiment for Hall B due to the large acceptance of CLAS. Further discussion of the setup and data selection is contained in the following two chapters. 


\section{CHAPTER 2}

\section{EXPERIMENTAL SETUP}

The data for this analysis were taken using the CEBAF Large Acceptance Spectrometer (CLAS) in Hall B at the Thomas Jefferson National Accelerator Facility (Jefferson Lab) in Newport News, VA. The primary mission of Jefferson Lab is to conduct research of the atomic nucleus at the quark level. The facility consists of an electron accelerator and three separate experimental halls: A, B, and C. Hall B is laid out as seen in Fig. 2.1 with the CLAS detector in the center. The detector is designed to take electroproduction data directly, but can also take real-photon production data using an $e^{-}$tagger that sits upstream, as shown in Figs. 2.1. The CLAS detector utilizes multi-wire drift chambers to reconstruct the momenta of final state particle leaving the target chamber. The detector is coupled with scintillator paddles used for timing information and particle identification. Additional calorimeters are used to identify neutral particles and improve $e^{-} / \pi^{-}$separation. For photoproduction, the photon tagger is used to measure the energy of the incident photon on the target as discussed in section 2.2 and shown in Fig. 2.4. 


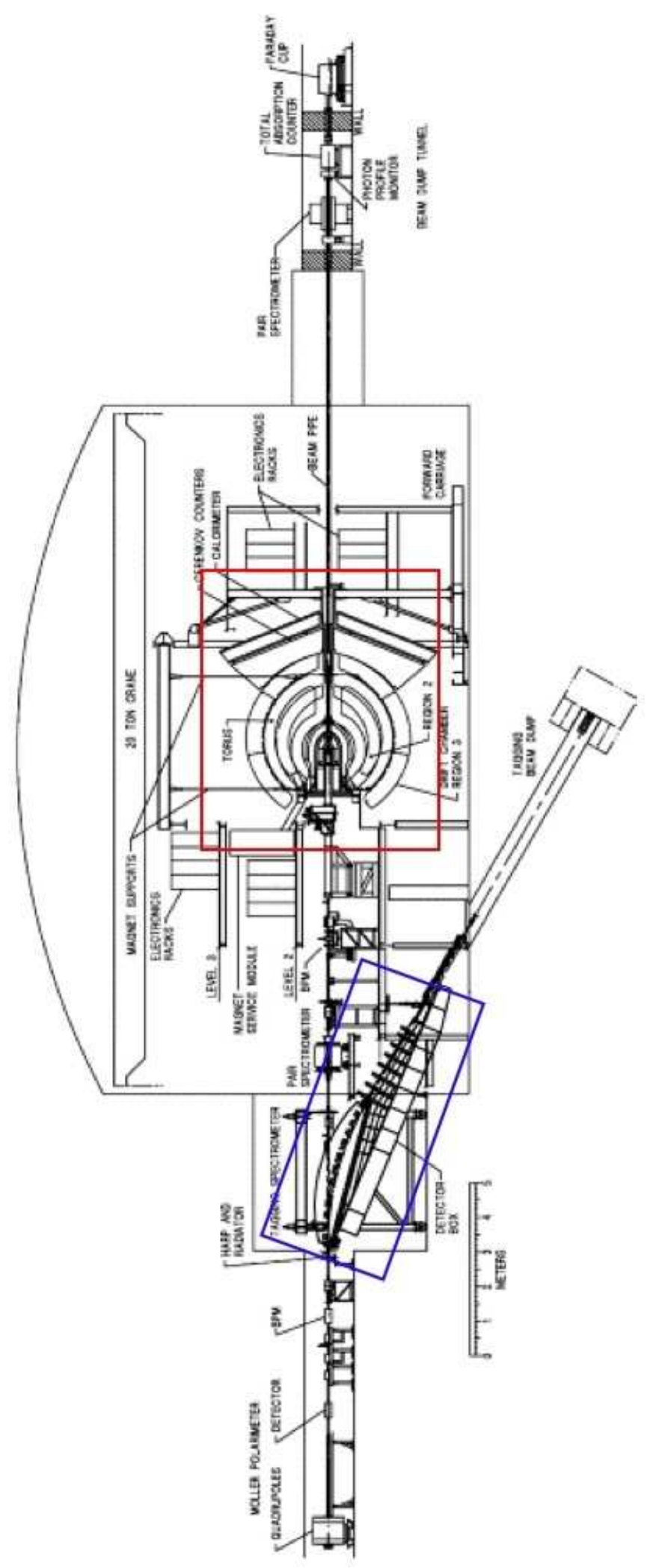

Figure 2.1: Hall B layout. Shows the relation of the beamline to the CLAS detector package, in the red box, and the tagger, in the blue box [18]. 


\subsection{The Continuous Electron Beam Accelerator}

The accelerator supplies a "continuous beam" of electrons consisting of regular pulses separated by $2.001 \mathrm{~ns}$ into Hall B. The accelerator supplies three times this rate to supply all three halls at any given time. It is an alternative to a beam with a much wider beam-burst separation of high intensity buckets. The beam is produced at the injector by an electron gun made of a gallium arsenide (GaAs) photocathode, an anode, and a system of lasers. The laser beam strikes the photocathode and knocks off electrons as a result of the photoelectric effect. The electric potential between the cathode and anode give the electrons an initial acceleration of $100 \mathrm{kV}$. Through the use of three different lasers, set with different frequencies, each of the three halls is able to receive beam energies and currents independent of the other two halls. This is very important for Hall $\mathrm{B}$ since its experiments require a current of a few $\mathrm{nA}$ while Halls $\mathrm{A}$ and $\mathrm{C}$ regularly operate using currents in the hundred $\mu \mathrm{A}$ range.

After leaving the injector the electrons are accelerated to $40-80 \mathrm{MeV}$ just before entering the first linear accelerator (LINAC). Acceleration is achieved using RF cavities made of superconducting niobium in a liquid helium bath. The temperature is maintained around $2 \mathrm{~K}$, at which the niobium exhibits superconducting properties, allowing for the propagation of electromagnetic waves with no resistance. The frequencies of the waves are tuned to give the electrons a series of boosts as they continue along, as seen in Fig. 2.2. After the initial acceleration, the electrons enter the north LINAC, which is made up of the same RF cavities as mentioned previously. At the end of the first LINAC the electrons are run through a Recirculation Arc and into the south LINAC. Electrons are steered through the $180^{\circ}$ turn by the use of a series

of dipole magnets. Since the beam may require multiple loops around the accelerator track, the beam is separated just before the Recirculation Arcs into different energy 


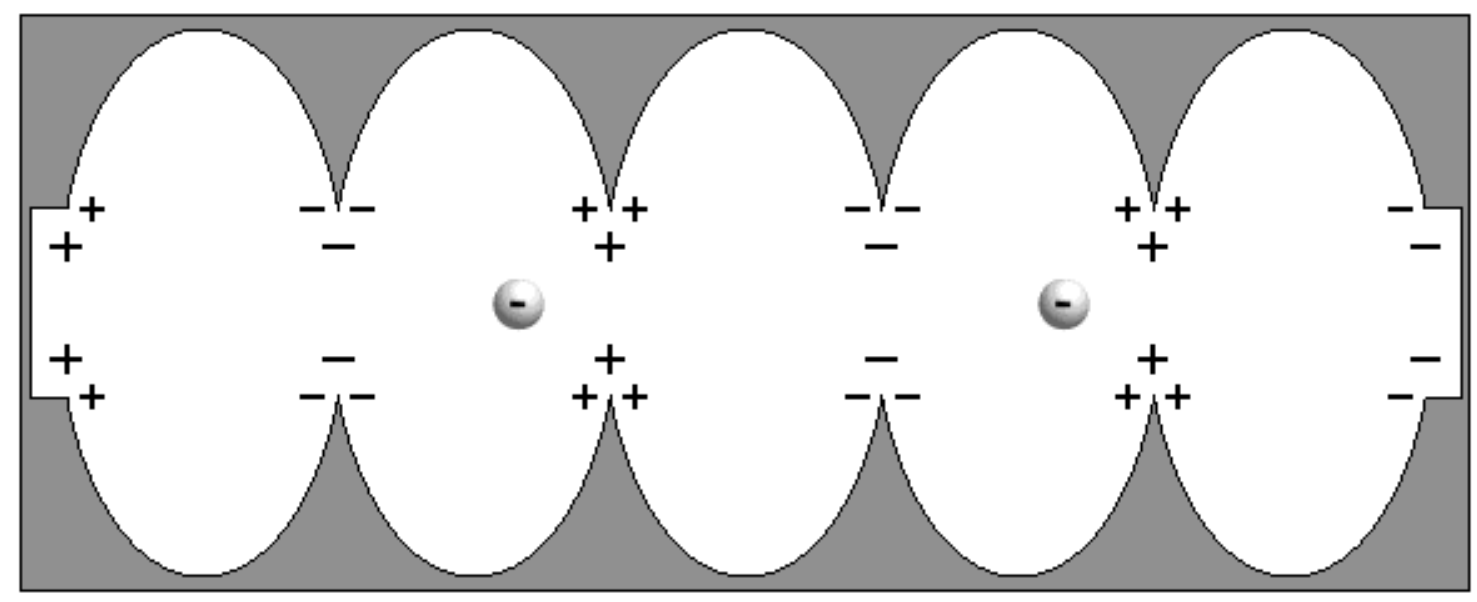

Figure 2.2: An illustration of electrons accelerated through RF cavity. The electrons are boosted when the RF waves are directed into the cavities and cause the electrons in the niobium metal to concentrate in certain areas. Since these areas have extra electrons, they become negatively charged. Other areas of the cavities have a depletion of electrons, so they become positively charged. The electrons in the beam are pulled towards the positively charged areas and are pushed away from the negatively charged areas.

sets corresponding to the number of laps the electron has made around the track. Each of these beam sets are then bent into the Recirculation Arc's dipole magnets that correspond to the kinetic energy of the electrons at that particular loop. In the current configuration, the maximum number of circuits is five. At the end of the arcs, the beam is recombined just before entering the next LINAC. After the beam is accelerated to the final kinetic energy, it is then split in the switchyard and routed into the three experimental halls. The layout of the accelerator is shown in Fig. 2.3. 


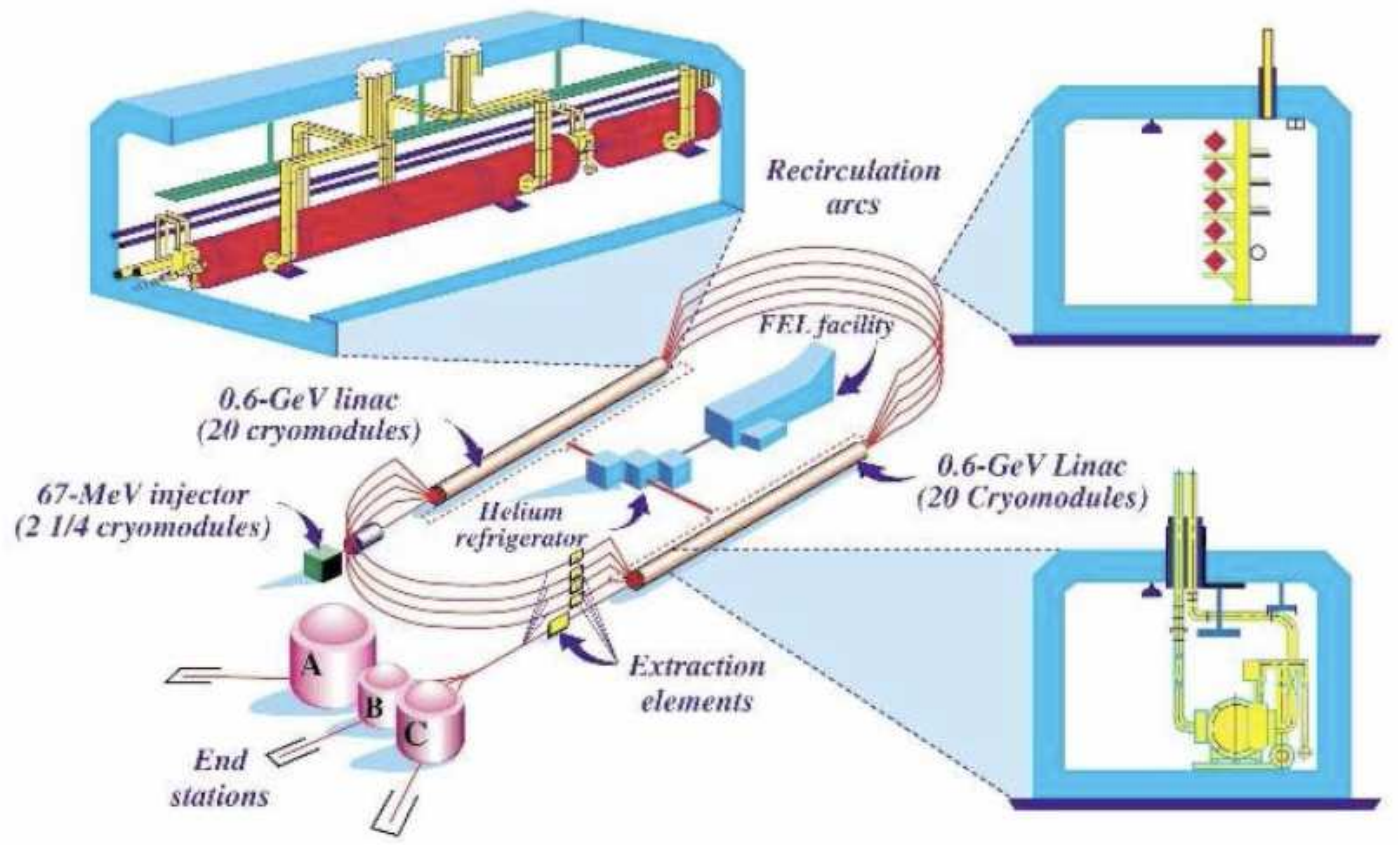

Figure 2.3: Layout of the CEBAF accelerator, [18]. The electron beam starts at the Injector and proceeds into the LINAC and Recirculation Arcs; where it will complete multiple loops until the electrons are at the energy needed in the experimental halls. When this happens, magnets at the Extraction Elements are used to bend to beam into the correct hall. 


\subsection{Hall B Photon Beam and Tagger}

A very thin radiator foil is used for photon beam experiments to scatter the incoming electron off of the electromagnetic field of a nucleus causing a photon to be emitted by bremsstrahlung radiation. The radiator assembly consists of several different gold and gold-plated foils mounted on an arm that is moved by a stepping motor to allow several thicknesses of radiator to be moved into the beamline. A $10^{-4}$ radiation length foil was used for this experiment. Three "harp" assemblies, having two thin wires are used to measure the electron beam profile and position and are located along the beamline. After any downtime or change in the beam, a harp scan is required and adjustments are made by the accelerator group to ensure more than $99 \%$ of the collimated beam goes through the target volume.

After traveling through the radiator, the recoil electrons and bremsstrahlung photons continue through a dipole "tagger" magnet that filters out the electrons. This dipole magnet's current is set by having the electron beam tuned to a target on the tagger beam dump. The current of the magnet is kept constant during the experiment for the photon tagging system to measure the energies of recoil electrons and to gain a coincidence signal for the CLAS detector. The tagging range is $20-95 \%$ of the electron's energy incident on the radiator, which corresponds to 1.14 to $5.45 \mathrm{GeV}$ for the $5.744 \mathrm{GeV} e^{-}$beam energy of the g12 experiment.

The photon tagger, in Fig. 2.4, consists of two layers of scintillator paddles. The first layer is the "E-plane" and is made up of 384 overlapping scintillator paddles that give the energies of the electrons. The paddles overlap by one third to produce an energy resolution of approximately $5 \mathrm{MeV}$ and are aligned perpendicular to the expected electron trajectories to minimize particle back-scatter. The second layer is

for timing, "T-plane," which is farther away from the dipole magnet to increase the 


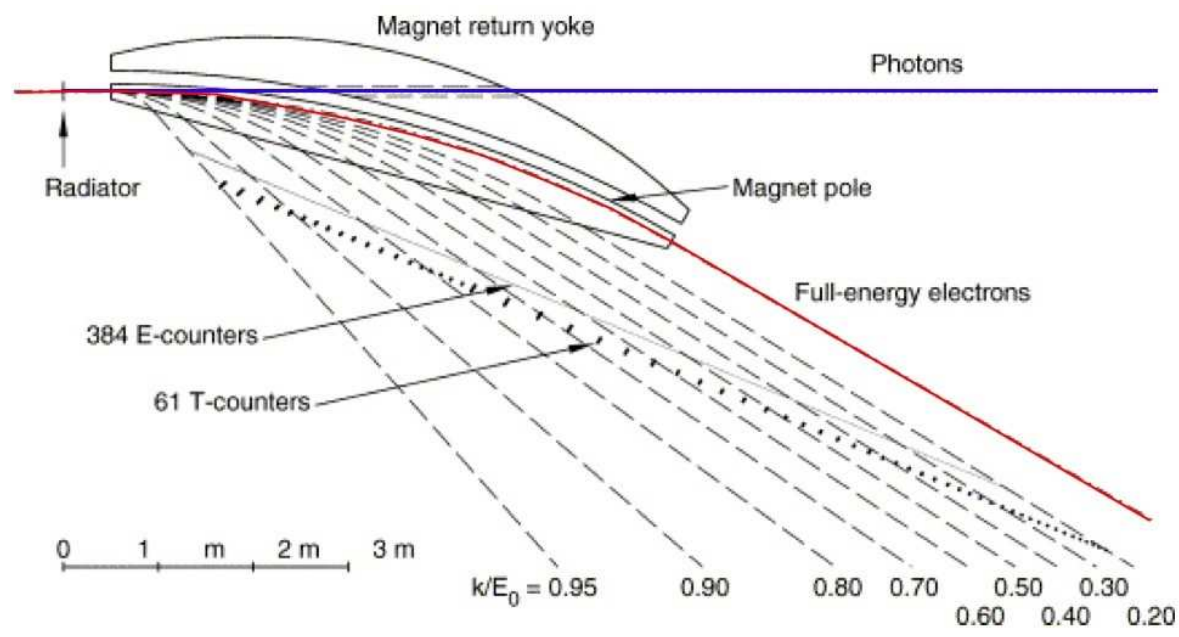

Figure 2.4: Side view of the tagger. The Tagger uses a dipole magnet to bend the electrons out of the photon beamline and into the E-Counters and T-Counters. The photon energy, $E^{\gamma}$, is then calculated from $E_{\text {beam }}^{e}-E_{\text {bent }}^{e},[18]$.

timing accuracy since the photomultiplier tubes (PMTs) connected to the scintillators are affected by magnetic fields. The T-plane is made up of 61 paddles overlapping by $10 \%$ so that there are no gaps in the coverage.

Once the electrons are bent out of the beamline, the photon beam passes through a $6.4 \mathrm{~mm}$ collimator upstream from the target followed by sweeping magnets. These sweeping magnets redirect any charged particles that may have been created by photons hitting the collimator out of the beamline and away from the detectors. Finally, the photon beam enters the 4-cm-diameter target with a beam spread of approximately $1 \mathrm{~cm}$ full width half maximum (FWHM).

\section{$2.3 \quad$ CLAS}

The photon beam interacts with the liquid hydrogen target that is kept at a temperature of $2 \mathrm{~K}$. For this experiment the target cell is $40 \mathrm{~cm}$ long and was located $90 \mathrm{~cm}$ upstream from the center of CLAS. The target placement provided more coverage of small-angle tracks than the nominal CLAS target position, decreasing the small angle 
acceptance from 8 to $6^{\circ}$. The change also reduces the maximum angle of acceptance from 140 to $100^{\circ}$. The layout of the CLAS detectors is shown in Fig. 2.5.

After the beam interacts with the target, the resulting spray of particles leave the target chamber and pass through the start counter on their way to the drift chambers. As part of the trigger, the start counter (ST) is designed to detect particles with high efficiency before the charged particles are influenced by the toroidal magnetic field. The counter timing is precise enough to decipher which beam bucket of photons interacted with the target at any given time. The start counter is made up of 24 2.2-mm-thick scintillator paddles with a single PMT at one end and was designed to have a timing resolution of 350 ps [19]. Fig. 2.6 shows the placement of the paddles around the target to form a hermetic hexagon around the target region.

The CLAS detector uses six superconducting magnets to produce a toroidal field to aid in charged particle identification. Under the effects of a magnetic field, charged particle tracks are bent under the force applied by the field with the particle's momentum given by $p=q r B$, where $r$ is the radius of curvature, $q$ is the charge of the particle, and $B$ is the strength of the magnetic field. The torus coils are cooled with liquid helium and produce a peak field of $3.5 \mathrm{~T}$ with a maximum operating current of 3790 A [20]. During this experiment, however, the current was at a half-field of $1930 \mathrm{~A}$ as a compromise to increase the acceptance of positive tracks and limit loss of track resolution.

The CLAS drift chambers are arranged into six wedge-shaped sectors in a hexagonal configuration around the beamline [21] as shown in Fig. 2.7. Each of these sectors consists of three regions. Region 1 is the innermost of the drift chambers and surrounds the CLAS target. Region 2 is located between the torus coils and is in an area of high magnetic field compared to regions 1 and 3 . Region 2 is used to determine the charge and momenta of the particles by measuring the radius of curvature 


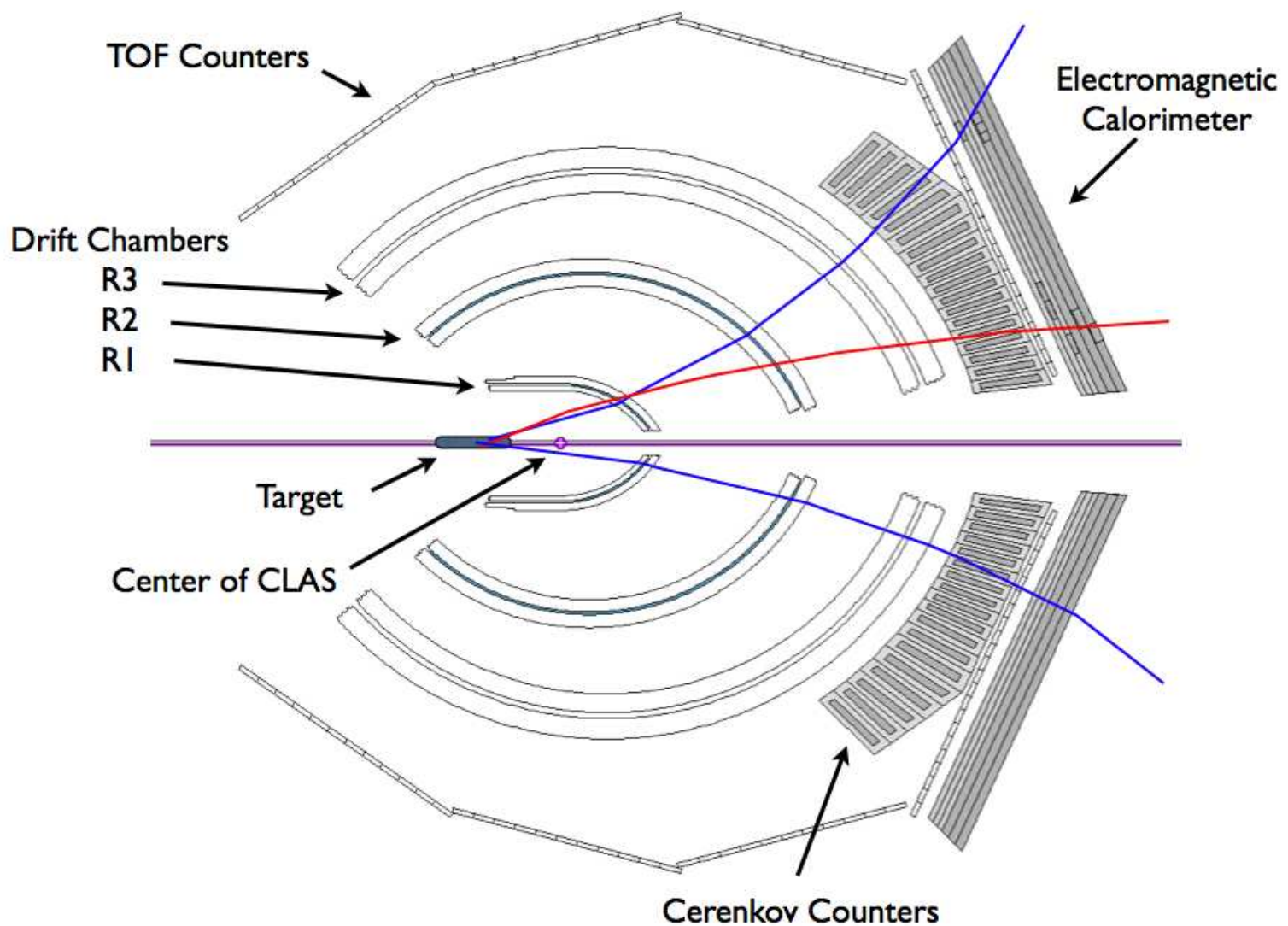

Figure 2.5: Side view of CLAS with a typical event overlaid to show the path of charged particles in CLAS. The blue tracks are a $p$ and $\pi^{+}$, the red track is a $\pi^{-}$. This is one event from the data skim, plotted with the CLAS event display program. The target (not shown to scale) is shown offset by $90 \mathrm{~cm}$ upstream of the nominal CLAS target position. 


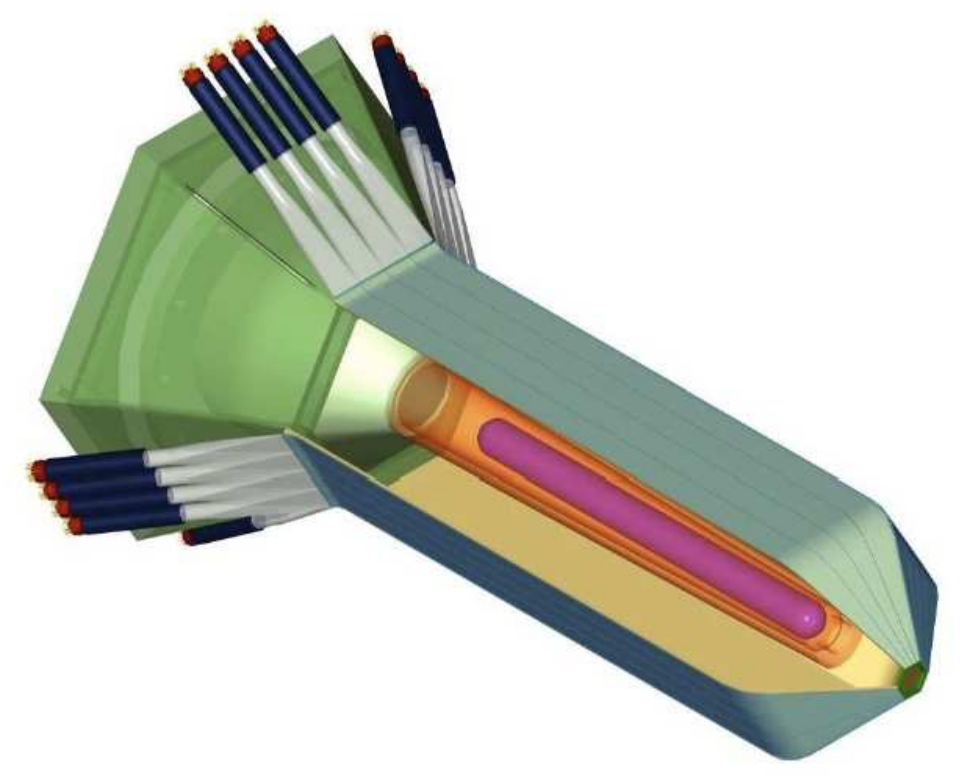

Figure 2.6: Diagram of the start counter [19]. The beam enters from the upper left of the image, into the target (purple) where the event originates. Outgoing particles pass though the scintillator paddles (blue) of the ST.

of the track. Region 3 surrounds these two regions and is outside of the torus. Each region is separated into two superlayers of six sense-wire layers, except for the first superlayer of Region 1 which has four sense-wire layers. The axial superlayer has its wires oriented perpendicular to the beamline and the stereo superlayer is tilted six degrees to provide azimuthal angle information in each region. The CLAS drift chambers (DC) are made up of approximately 30,000 sense wires wires. The sense wires are maintained at a positive potential of about $1.4 \mathrm{kV}$. Each sense wire is surrounded by six field wires, as shown in Fig. 2.8, with a negative potential of $-0.6 \mathrm{kV}$ [21]. The gas surrounding the wires is composed of $88 \%$ argon and $12 \%$ carbon dioxide. When a charged particle passes through the drift chamber, the gas becomes ionized causing freed electrons to drift toward the sense wires, creating a pulse in the wire that is amplified and recorded after signal discrimination. The timing only gives the radius for the drift distance. The tracking algorithm works by initially using a table 


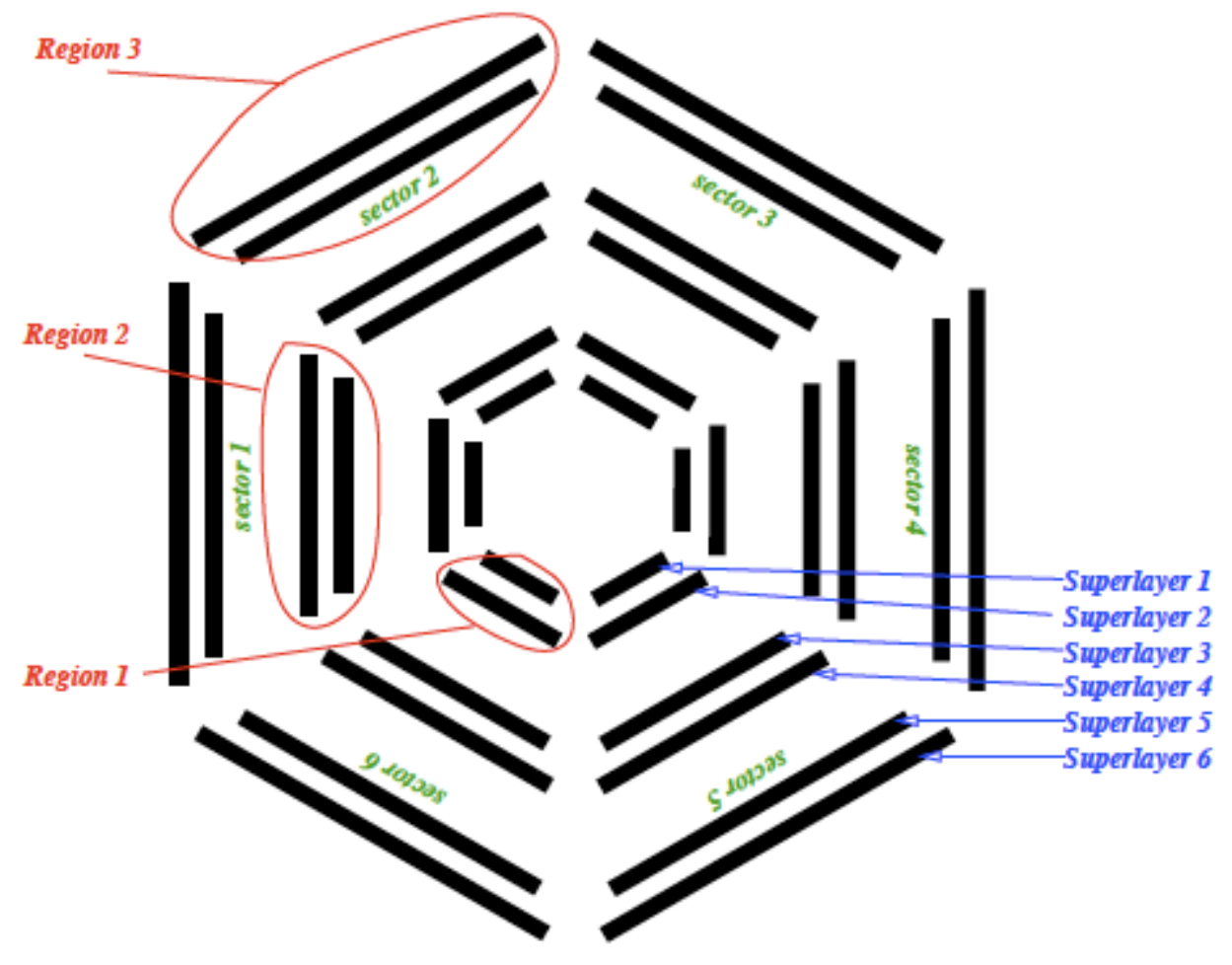

Figure 2.7: Diagram of the regions and super layers of the DC [21] looking downstream.

to convert timing to drift distance. By fitting the tracks and adjusting the fitting parameters to minimize the residuals, the track reconstruction is improved.

Surrounding the region 3 DC are the Time-of-Flight (TOF) detectors, which record the total flight time of the particle coming from the target. The detectors are made up of 5.08-cm-thick scintillator paddles with PMTs attached to both ends [23]. The scintillators are positioned perpendicular to the average flight path of the local particle tracks (Fig. 2.9). The entire active range covers $8^{\circ}$ to $142^{\circ}$ of the polar angle and covers the DC hermetically. The time resolution is dependent on the size of the paddles. For the larger paddles the path length from the track vertex to PMTs is larger, and the resolution ranges from 80 to 160 ns. For g12, the TOF was used to identify the number of possible tracks in the event trigger. 


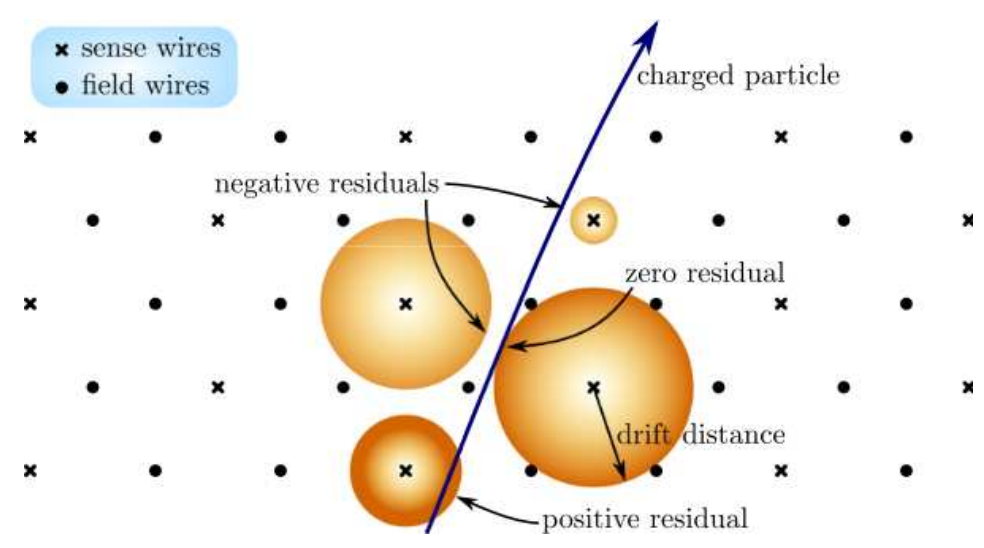

Figure 2.8: View parallel to the DC wires. Shows the residuals in relation to the drift time [22].

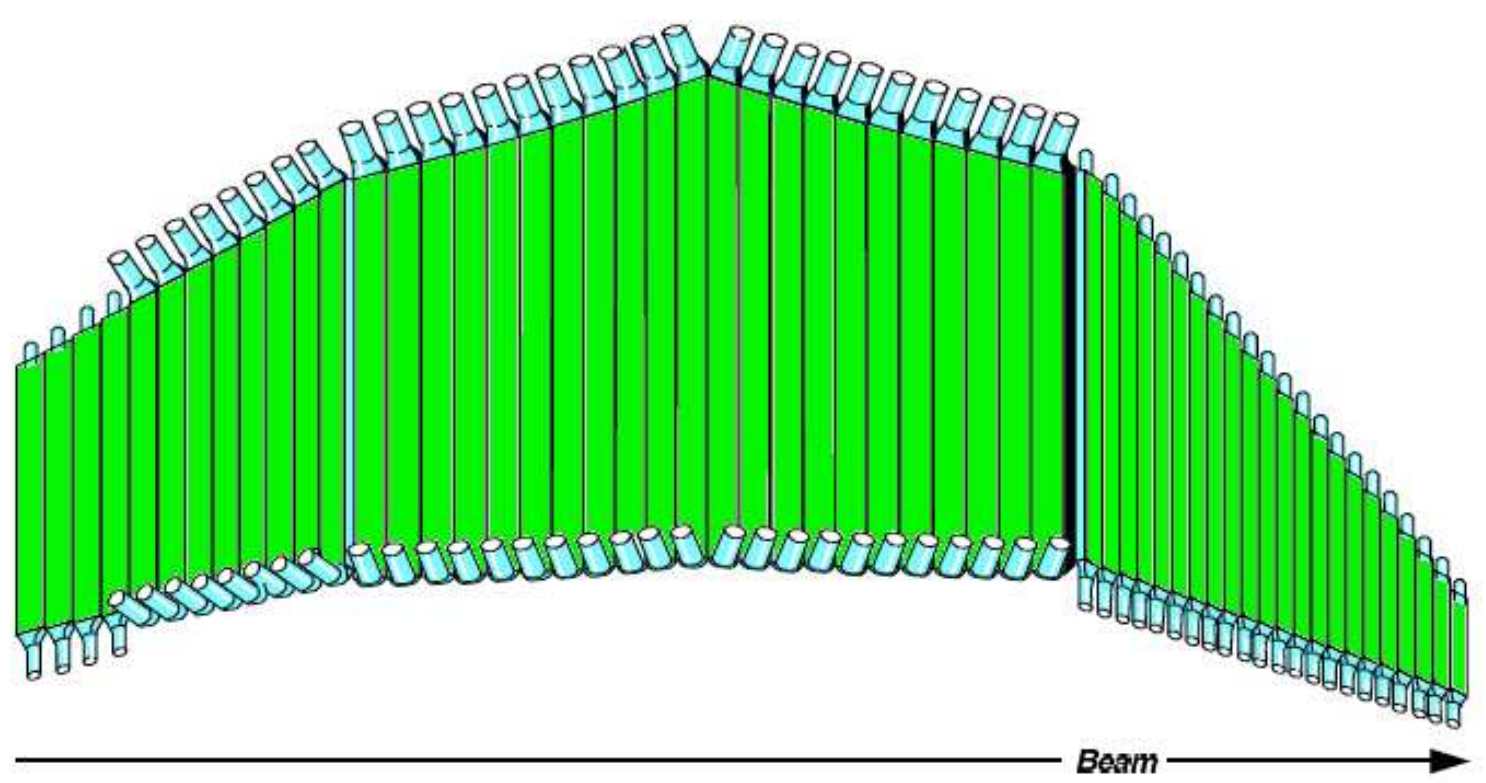

Figure 2.9: Diagram of one sector of the TOF paddles [23]. The green regions are the placement of the scintillator paddles and the blue ends show the positions of the light guides and PMTs. 


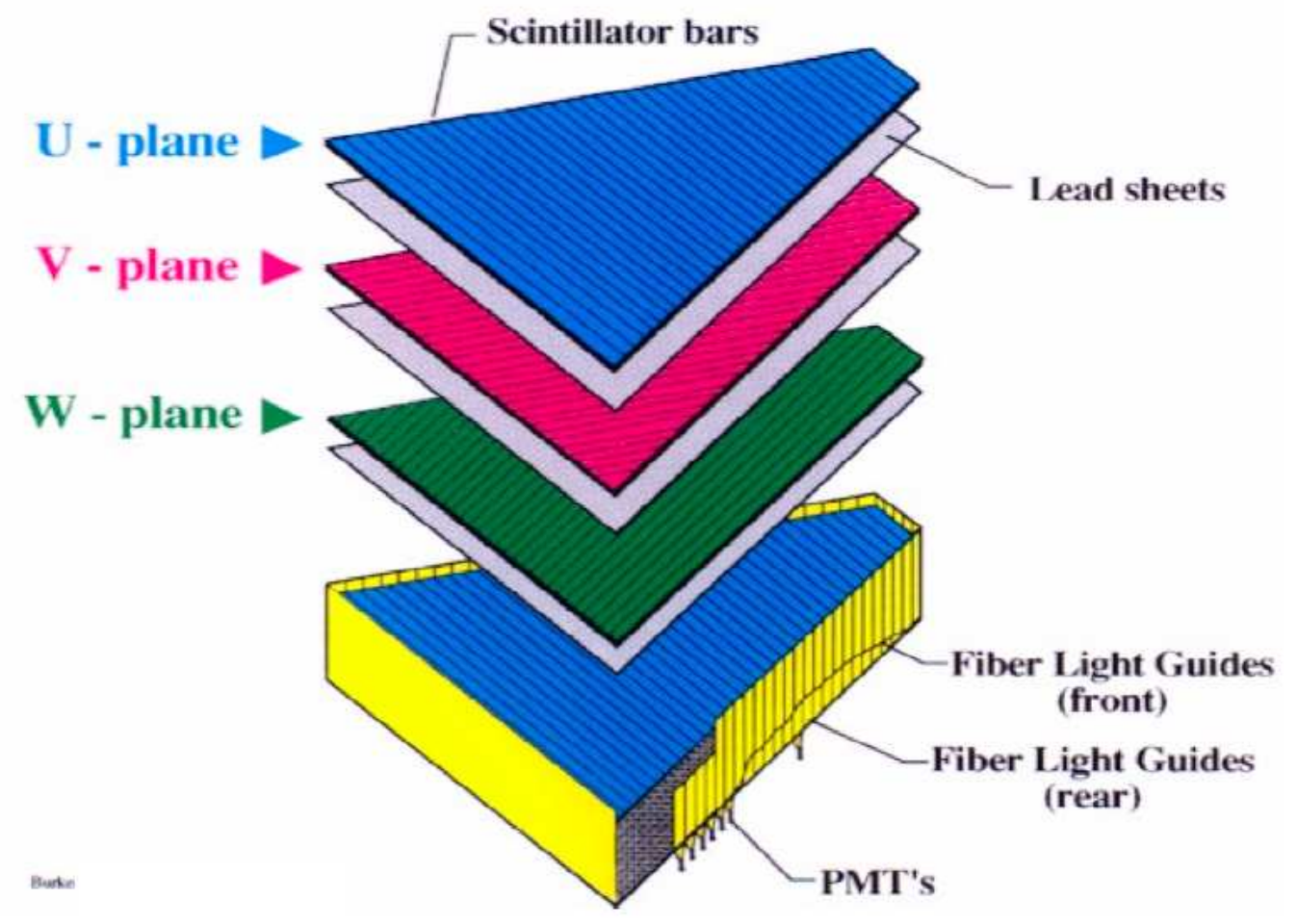

Figure 2.10: Electromagnetic Calorimeter sectional view [24]. A neutral particle will pass through the scintillator undetected but will interact with the lead sheets causing a cascade of radiation that will be detected with the subsequent scintillator layers.

The forward Electromagnetic Calorimeter (EC) is used to detect neutral particles and to separate $e^{-} / \pi^{-}$in electron experiments. It covers a range in polar angle up to $45^{\circ}$ [24]. The detector is made up of 39 alternating scintillator layers and lead sheets. Each layer consists of 36 strips of scintillator that are rotated $120^{\circ}$ every 13 layers, giving three views of the particle at the point of intersection (see Fig. 2.10). The EC mass resolution for reconstructing $\pi^{0}$ and $\eta$ from $\gamma \gamma$ is $\delta m / m=0.11-0.14$ [24].

The inner calorimeter's hodoscope (IC) and cherenkov counters (CC) were in use for this run period but are not part of this analysis. The $\mathrm{CC}$ is used for electron identification. The IC hodoscope is a matrix of scintillator paddles covering the extreme small angles. The IC is used to detect charged and neutral particle and discriminate between $e^{-}$and $\pi^{-}$. 


\subsection{Hall B Data Readout}

In order to minimize computer deadtime (the amount of time lost to the process of recording data while still receiving events of interest) a two-level trigger system was used. For level 1, the signals from the SC PMTs were all processed in 90 ns and sent to the Trigger Supervisor (TS). The resulting signal was used as a start signal for the PMT TDCs and ADCs, as well as a common stop signal for DC signals (after a delay). Once the stop signals were given, all the signals were digitized and read out. The level 2 trigger sorts the signals into probable tracks that were then sent to the data acquisition (DAQ) system. The level 2 trigger selects tracks to be a coincidence of the ST and TOF in the same sector. From there the main production trigger used by the g12 experiment was a coincidence of two charged tracks in different sectors and coincident with a tagged photon above $4.4 \mathrm{GeV}$. The CLAS DAQ is composed of the Event Builder (EB), Event Transport (ET), and Event Recorder (ER). At the EB, the signals are assembled into whole events. These events were given an event type, event number, and run number. These events were stored by the ET system that manages the shared memory and picked up by the ER to be written to permanent storage.

\subsection{Detector Calibration and Particle Identification}

The goal of calibration is to adjust calibration constants and detector alignment in the reconstruction software and to find timing and energy information for each detector system. For CLAS experiments, the detector calibrations are separated by the subsystems: tagger, TOF, DC, EC, and CC. The first three of the calibrations are crucial to the basic charged particle reconstruction. For this experiment, the tagger

was calibrated by Mukesh Saini, the TOF was calibrated by Craig Bookwalter, the 
DC timing was calibrated by the author, and the DC alignment was done by Burnham Stokes.

\subsubsection{Tagger Calibrations}

CLAS particle reconstruction is dependent on the detector timing and directly affected by the tagger time. For photon runs, the tagger signal is used with the radio-frequency time $(\mathrm{RF})$ of the accelerator beam to establish a timing reference. Once calibrated, the tagger and RF times are corrected for phase differences [25]. With the tagger calibrated, the closest RF corrected tagger time corresponding to the the smallest

$d t$ between $t_{\text {pho }}+t_{\text {prop }}$ and ST vertex time defines the event start time. The timing resolution of the tagger is 130 ps after calibrations. The energy calibrations of the tagger were done using the exclusive channel $p \pi^{+} \pi^{-}$. The tagged photon energy was adjusted by the equation:

$$
E_{\text {beam }}=E_{p}+E_{\pi^{+}}+E_{\pi^{-}}-m_{p}
$$

where $E_{p}, E_{\pi^{+}}$, and $E_{\pi^{-}}$are the energies of the outgoing particles and $m_{p}$ is the target mass. After the initial beam calibration, additional corrections are used to refine the values later in the kinematic fit discussed in Section 3.6. After calibrations, the average incident photon energy resolution for the g12 run period was $5.6 \mathrm{MeV}$ as detailed in Ref. [22].

\subsubsection{Start Counter and Time-Of-Flight}

The calibrations of the ST and TOF system involve calibrating the signals and aligning the scintillator paddles [23]. First, the raw TDC times $(T)$ are converted into hit times by

$$
t=c_{0}+c_{1} T+c_{2} T^{2}+t_{w a l k}
$$


where $c_{0}, c_{1}$ and $c_{2}$ are the constants to be determined. The $t_{\text {walk }}$ is the time walk correction due to pulse height variations and is determined differently for the ST and TOF.

The timing of the start counter (ST) is used to determine the tagger hit associated with the physical event. Exclusive $p \pi^{+} \pi^{-}$events were used and the tagger hit was matched to the average vertex time measured from these final state particles. The time walk $\left(t_{\text {walk }}\right)$ of the signal from the track to the PMT is determined by the equation:

$$
t_{\text {walk }}=t_{0}+\frac{t_{1}}{a-a_{0}},
$$

where $t_{0}$ and $t_{1}$ were determined for each paddle from the data for $A D C_{\min }<a<$ $A D C_{\text {max }}$. Here, $a$ is the ADC signal and $a_{0}$ is the ADC pedestal value. The final resolution of the ST was approximately $370 \mathrm{ps}$, which is sufficient to identify the $2 \mathrm{~ns}$ beam bucket associated for the event.

The TOF is used along with momentum information from the DC to calculate the mass and charge of the particles. The method for determining the TOF resolution is identical to that of the start counter. However, the time walk correction is more sophisticated because of the finer resolution of the TOF:

$$
\begin{aligned}
t_{\text {walk }} & =b x^{-c} & & : a<a_{1} \\
& =\frac{b}{a_{1}^{c}}\left(b+c\left[1-\frac{\left(a-a_{0}\right)}{a_{1} V_{T}}\right]\right) & & : a \leq a_{1},
\end{aligned}
$$

where $t_{0}, b$ and $c$ are constants determined for each TOF paddle and for each calibration run range, $a$ is the TOF ADC signal, $a_{0}$ is the ADC pedestal value and $V_{T}$ is the discriminator threshold value. This equation is essentially a power law below some ADC value $a_{1}$ and a linear function above, and it has the property of being smooth at this transition point. The timing of the left and right PMTs needs to be aligned. 
The average time between the two PMTs for a paddle is

$$
T=\frac{T_{L}+T_{R}}{2} .
$$

For the case of only one TDC signal, the time is calculated by

$$
T=T_{L, R}-\frac{y}{v_{L}} .
$$

where $y$ is the distance along the paddle to the PMT from the point where the tracking determined the particle entered the scintillator. The TOF had a timing resolution of approximately $230 \mathrm{ps}$ after all calibrations were completed [22].

\subsubsection{Drift Chambers Calibration and Alignment}

In the DC there are 34 layers and for one track about 30 layers will fire because of detector inefficiency and holes in the detector. Each hit fired in the DC is used to determine the track by using a least squares fit in the track reconstruction program. The distance from the fitted track to the sense wire is the distance of closest approach, DOCA. The calculated distance from the sense wire to the track is known as DIST, which is calculated from the drift time information that is adjusted in the calibrations. The difference between DOCA and DIST is the residual, RESI, and is related to the resolution of the DC. Track reconstruction using the DC works in two stages. Stage

one is the "hit-based" tracking, where the hit wires are connected to form a track. This stage forms the initial guess for the second stage. The second stage is the "timebased" tracking. By taking the time of flight, from the TOF and ST detectors, the initial wire times are converted to DIST using the calibrated drift velocity parameters $[21]$.

To calibrate the DC parameters, DIST is calculated from the initial drift velocity parameters that are taken from the previous experiment for an initial guess. The 
DIST is then fit for the DOCA to return a new set of parameters. The process is reiterated until the drift velocity fit parameters converge, then the parameters are given final adjustments by fitting the residuals. The quality of the DC calibration was checked by plotting the weighted mean and sigma of the double-gaussian fits to the residual distributions against the run number. The run by run results of the fits are in Figures 2.11 and 2.12, where the final resolution is $380 \mu \mathrm{m}$ with a momentum resolution of $\delta \mathrm{p}=0.002 \mathrm{GeV}^{-1} \mathrm{p}^{2}$. After calibrations, the probability of a charged track reconstructed in the DC is 95 to $98 \%$. This result was obtained by taking a data sample from the g12 run period and running the data through the event viewer.

Prior to this experiment the DC had undergone repairs to a sector in Region 3 that involved removing and replacing the detector. To account for the new position of this particular region, its hit resolution tolerance was magnified (multiplied by a large number) to allow the reconstruction to find the track. Then the alignment software moved the position of the chamber in the software around until $\chi^{2}$ was minimized for the tracks. It was assumed region 1 and 2 were already aligned for this sector. Figure 2.13 shows an example of how the alignment initially started and the final result after pass 3 of the calibration software. 


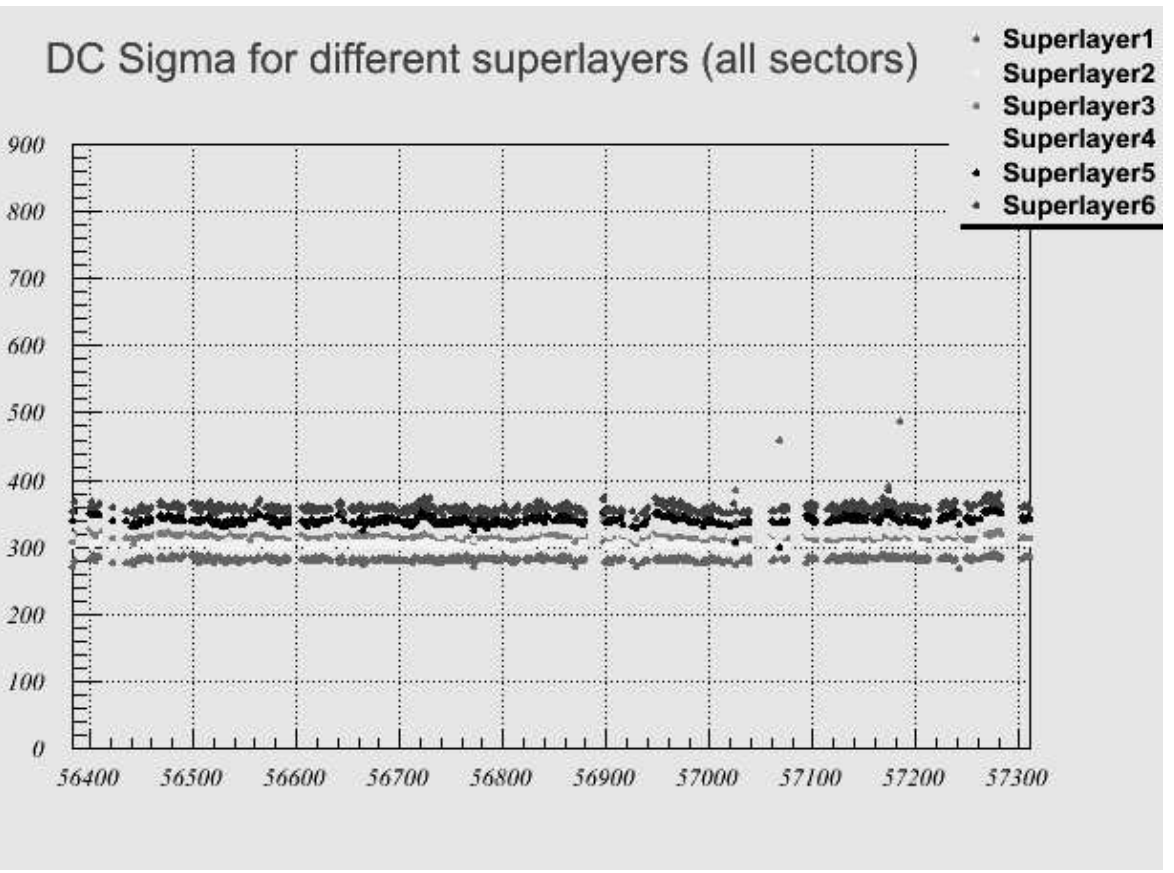

Figure 2.11: Run by run plot of the sigma from the double gaussian fit on the residual fit of the DC. Sigma is the physical resolution of the chamber.

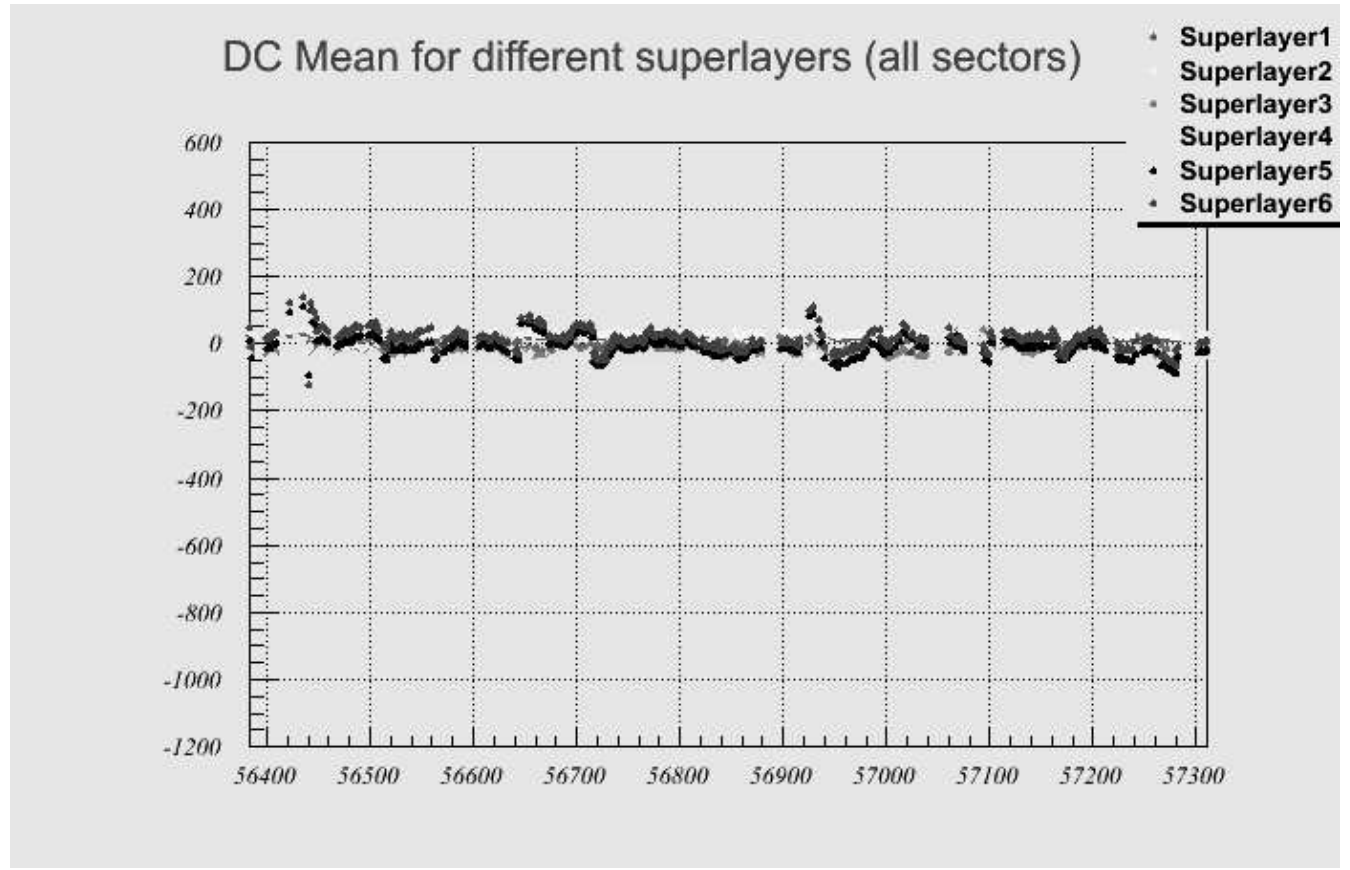

Figure 2.12: Run by run plot of the mean from the double gaussian fit on the residual fit of the DC 

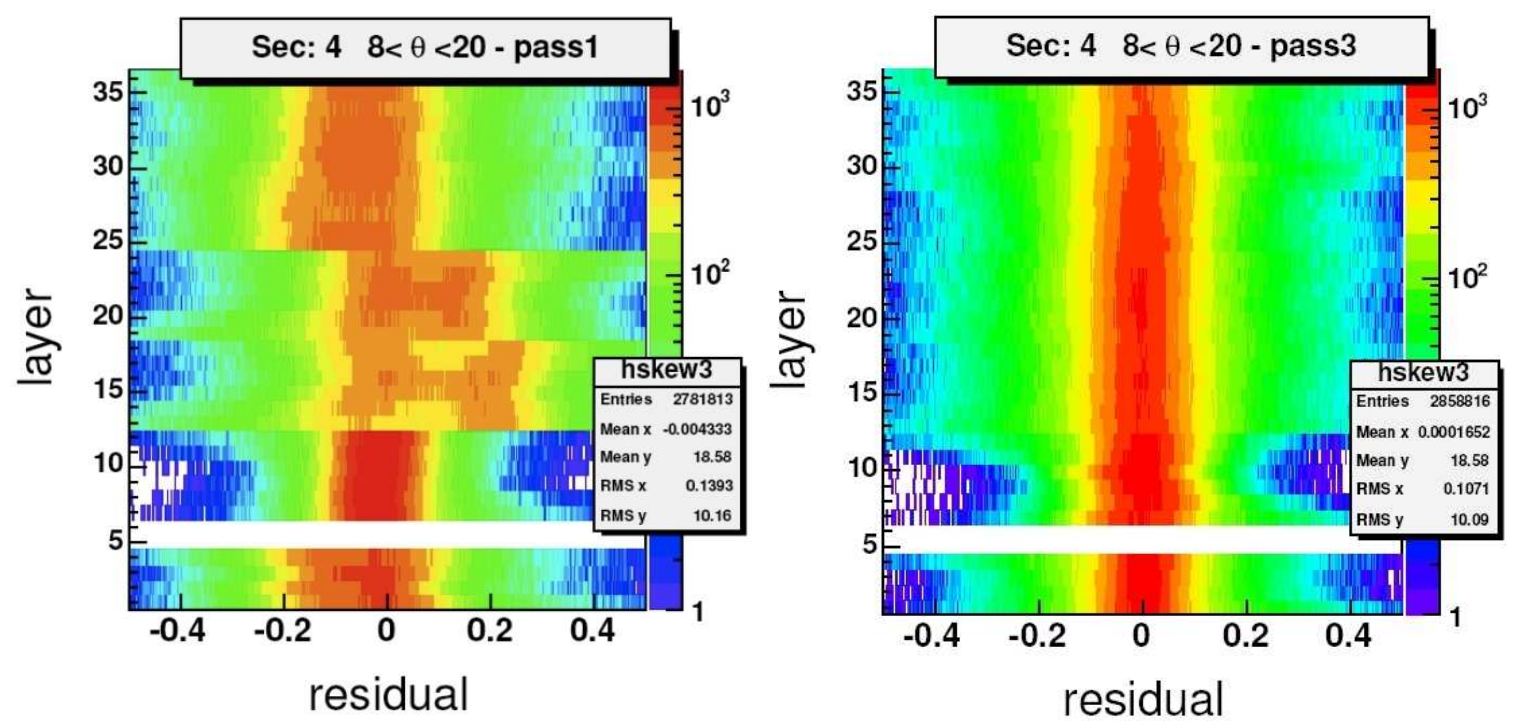

Figure 2.13: Alignment of sector 4, before and after the final DC alignment in software. Each region is made of two super layers with 6 layers of wires, except the first super layer only has 4 layers of wires. The wire layers 1 to 12 are region 1 , layers 13 to 24 are region 2 and layers 25 to 36 are region 3 . In the plot on the left, the alignment is consistent over individual regions but region 2 is poorly aligned and the three regions are not aligned with each other. After alignment, right plot, the residuals are in line over the whole sector. The residuals are in units of $\mathrm{cm}$ in these plots. 


\subsection{Data Reconstruction}

The written event information consists of raw TDC and ADC values from the CLAS detector and the tagger and is converted by the DAQ into un-calibrated banks in BOS format. The BOS format is the standard output for CLAS data. Once the raw data are written, the raw BOS files are processed by the event reconstruction program in offline analysis. Event reconstruction uses the detector geometry parameters and calibration constants to reconstruct the particle tracks in the events. Figure 2.14 shows in detail the process of "cooking" data. The final output is written into the cooked data banks, and includes beam reconstruction, charged particle tracks, momentum measurements, event vertex fitting, event timing, and preliminary particle identification. The cooking of the g12 data set was overseen by Johann Goetz of UCLA and was finished in December, 2009.

\subsubsection{Particle Identification}

To identify a charged particle, both the tracking and timing information is used. Given that the momentum of a charged particle is equal to the product of the radius of curvature in region 2 of the DC $(r)$, the particle's charge $(q)$, and the strength of the magnetic field $(B)$; a charged particle's tracking information is used to determine the momentum $(p=r q B)$ of the particle. The TOF information is used with the flight path information to determine the velocity $(\beta)$. The mass of the particle is calculated in combination using $m=p / \beta \gamma$ and the reconstructed particle is given a particle ID on the basis of the calculated mass.

The CLAS detector package has limited neutral particle detection. One technique to reconstruct neutrals is to take the known charged particle tracks and reconstruct

the missing momentum. This works well for reactions with a single neutral particle, 


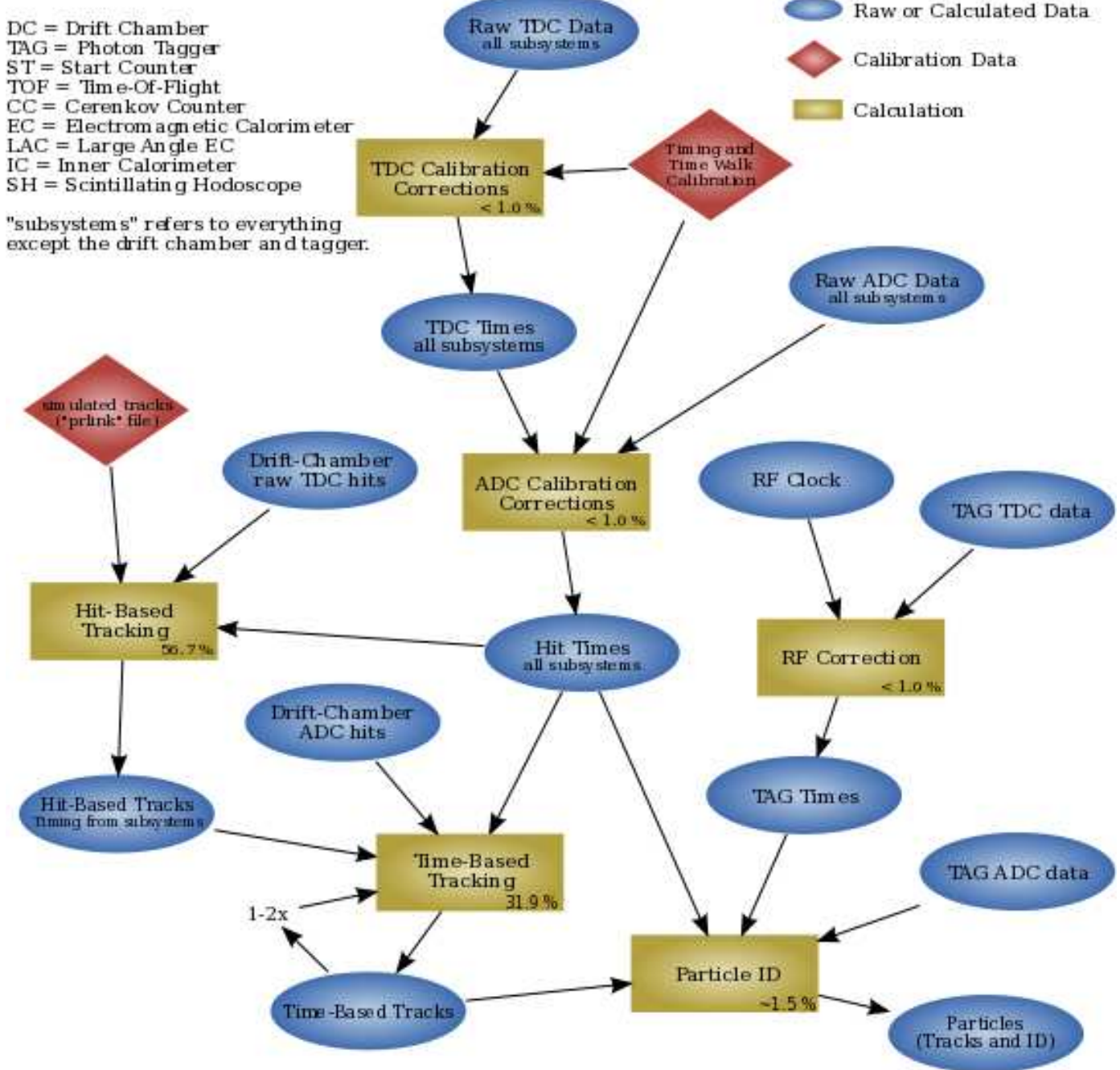

Figure 2.14: Flow chart of reconstruction process for CLAS. 
however for multiple neutrals this is not possible. Actual detection of neutrals has a lower efficiency than for charged particles and is roughly $10 \%$ for a single photon while between 30 to $60 \%$ for a positively charged track. The neutral particle signal used in the following analysis comes from the electromagnetic calorimeter. When a neutral particle goes through CLAS, it passes through the ST, DC, and TOF without depositing any detectable amount of energy. 


\section{CHAPTER 3}

\section{DATA ANALYSIS}

The reaction in this analysis is:

$$
\begin{array}{r}
\gamma p \rightarrow \Delta^{++} X \\
X \rightarrow \eta \pi^{-} \\
\Delta^{++} \rightarrow p \pi^{+}
\end{array}
$$

where the detected particles are the $p \pi^{+} \pi^{-}$. The missing particle, $\eta$, is reconstructed from the four-momenta of the detected charged particles $\left(P_{p}, P_{\pi^{+}}\right.$, and $\left.P_{\pi^{-}}\right)$and the tagged photon energy $\left(P_{\text {beam }}\right)$ by the following formulas:

$$
P_{\text {Miss }}=\left(P_{\text {beam }}+P_{\text {target }}\right)-\left(P_{p}+P_{\pi^{+}}+P_{\pi^{-}}\right)
$$

and

$$
M=\left|P_{\text {Miss }}\right|
$$

where $P$ is the four-momentum and $M$ is the missing mass. The $\eta$ decays to two $\gamma$ with a branching ratio of $39.31 \%$. The second and third most probable decay channels are to $3 \pi^{0}(32.52 \%)$ and $\pi^{+} \pi^{-} \pi^{0}(22.74 \%)$. Requiring both of the photons from the $\eta$ decay to be detected with CLAS in the full reaction $\gamma p \rightarrow p \pi^{+} \pi^{-} \gamma \gamma$ or $\gamma p \rightarrow p \pi^{+} \pi^{-} \gamma \gamma(2 \pi)$ dramatically reduces the acceptance by $90 \%$ per detected photon. The initial event selection requires events with one or more $\gamma$ but to increase the signal to background ratio, two photons are required. This chapter will discuss the cuts and the additional corrections to the data.

\subsection{Event Vertex and Beam Energy Cut}

The target used in this experiment is $40 \mathrm{~cm}$ long and is centered at $90 \mathrm{~cm}$ upstream from the center of CLAS. The vertex of the event is calculated from distance of closest 
approach (DOCA) of two charged tracks and the vertex is the midpoint of the vector DOCA. Selected events have a vertex position of $-110 \mathrm{~cm}<z<-70 \mathrm{~cm}$ as seen in Fig. 3.1(c). The allowed vertex radius is $\sqrt{x^{2}+y^{2}}<5 \mathrm{~cm}$ to ensure the track originated from inside the target cell.

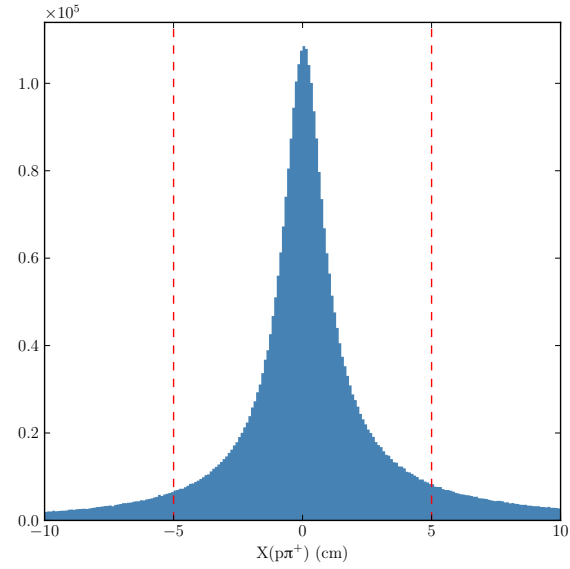

(a) $x$ vertex distribution

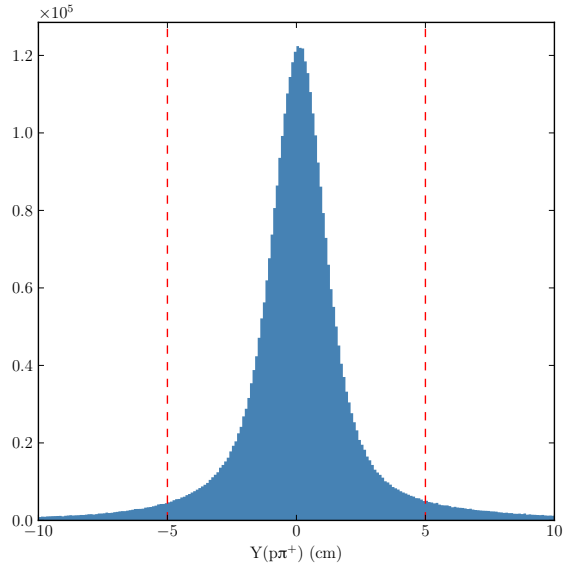

(b) $y$ vertex distribution

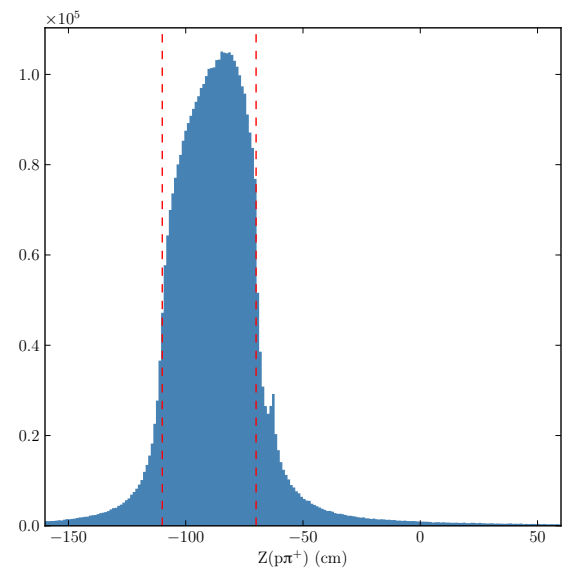

(c) $z$ vertex distribution

Figure 3.1: The $x, y, z$ coordinate plots of the $p \pi^{+}$intersection. Taken from the particle skim of $p \pi^{+} \pi^{-} \gamma \gamma$. The dashed red line shows the cut used on the data.

For this data set, a cut on the photon beam energy is implemented to enhance peripheral meson production and to match the trigger configuration used during the run period. The ideal trigger conditions are based on a combination of the calculated 


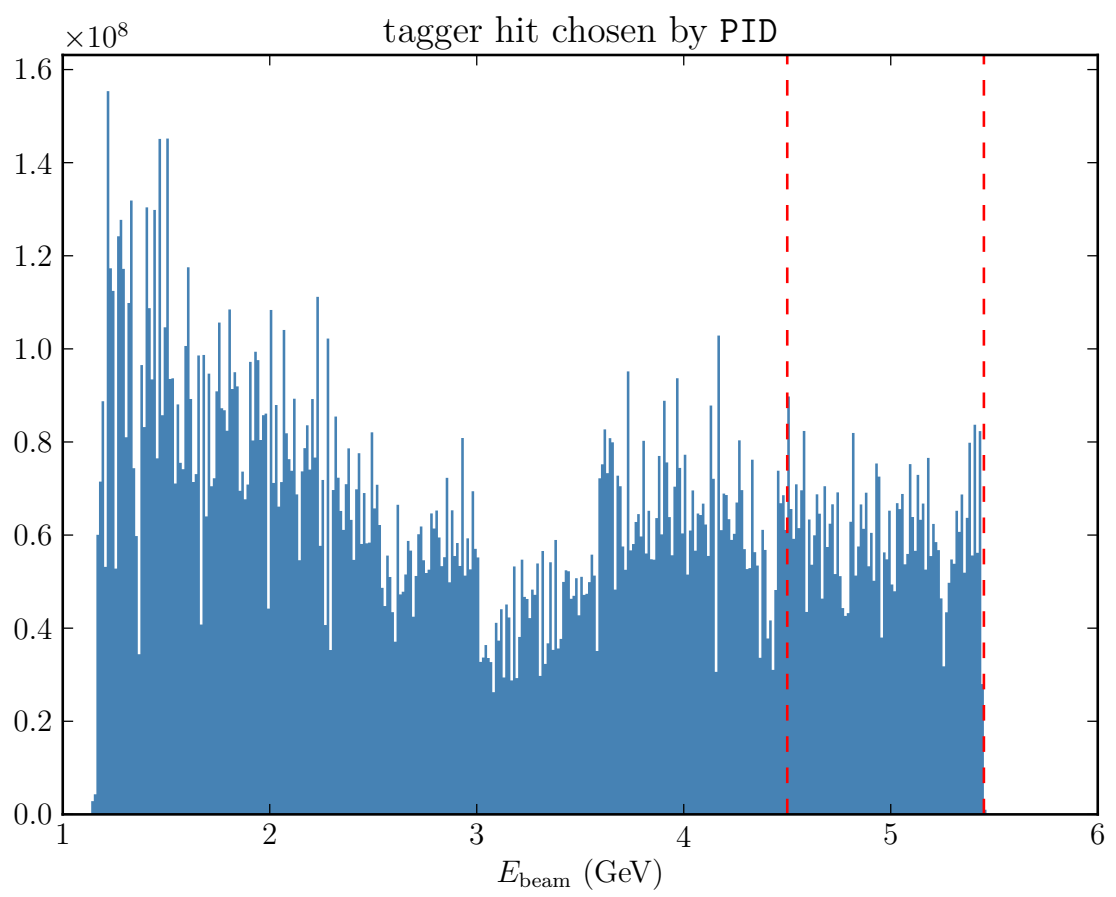

Figure 3.2: The photon beam energy. The accepted range is $4.45 \mathrm{GeV}<E_{\gamma}<5.45$ $\mathrm{GeV}$. The cut used in this analysis is shown by vertical dashed lines.

production thresholds for the desired decay channels and the background hadron production. For $\gamma p \rightarrow \Delta^{++} X$, where $X$ is the $a_{2}(1320)$, the minimum photon beam energy is $2.85 \mathrm{GeV}$ from

$$
E_{\gamma}=\frac{m_{X}^{2}+m_{r}^{2}-m_{t}^{2}+2 m_{X} m_{r}}{2 m_{t}}
$$

where $m_{X}$ is the resonance, $m_{r}$ is the recoil baryon, and $m_{t}$ is the target. The energy range, shown in Fig. 3.2, is set to be $4.45 \mathrm{GeV}<E_{\gamma}<5.45 \mathrm{GeV}$, where $E_{\gamma}$ is equal to the difference in the energy of the electron beam entering the hall and the energy of the electron detected in the tagger $\left(E_{e}-E_{e^{\prime}}\right)$. When there is more than one photon in the same time bucket, the photon with the highest energy is selected because the beam energy cut includes only the high energy range. For events with one photon within the energy cut, $12.9 \%$ of the events have a second photon within the beam timing bucket, as shown in Fig. 3.3 (left), and $2.19 \%$ of events where the second 

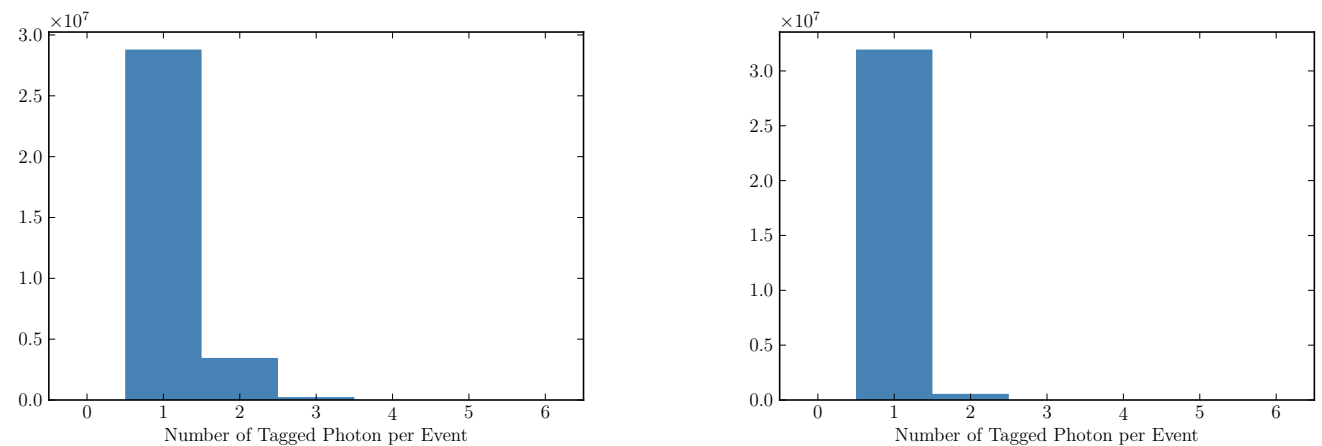

Figure 3.3: The left plot is the number of tagged photons in an event within the timing window. The right plot is the number of tagger photons in the timing window and in the beam energy selection.

photon is within the beam energy selection, as shown in Fig. 3.3 (right). The majority of the events with the wrong photon will fail the additional data selection cuts due to the dependence of the $\eta$ selection on the missing mass off of the charged tracks, which is in turn dependent on the beam energy.

\subsection{Timing Cut}

The start time of an event is determined by the tagger and ST, while the flight time of charged particles is measured by the TOF counters. The time of the event at the vertex, $t_{v t x}$, is calculated two ways; first from the RF-corrected tagger time, $t_{v t x}(\mathrm{TAG})$, and second from the ST, $t_{v t x}(\mathrm{ST})$. The events with correctly identified beam buckets will have a $\Delta t_{v t x}$ within \pm 1.002 ns. Here $t_{v t x}(\mathrm{TAG})$ is $R F-t_{\text {prop }}, R F$ is the RF-corrected time that the photon crossed the center of the target and $t_{\text {prop }}$ is the propagation time from the center of the target to the track's vertex $z$-coordinate. The difference in vertex times for a given event is selected to be within the beam pulse separation time (2.004 ns), as shown in Fig. 3.4.

Using the particle identification, the $t_{v t x}$ (TOF-PID) is calculated from

$$
t_{v t x}(T O F-P I D)=t_{T O F}-\frac{l_{T O F}}{c \beta_{P I D}}
$$



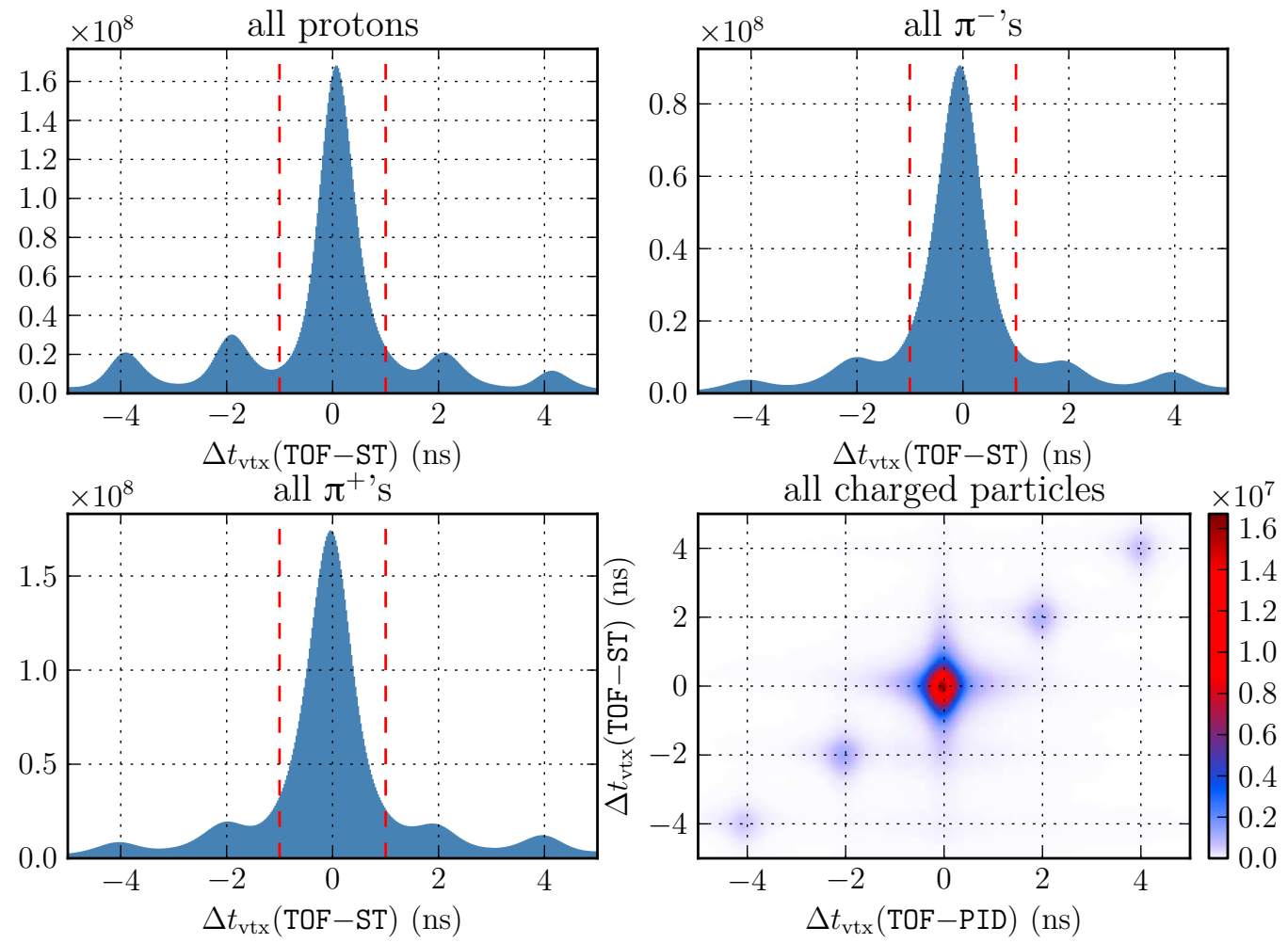

Figure 3.4: Plots of the difference in start counter vertex time to RF-corrected tagger vertex time in the upper left, upper right, and lower left. The lower right plot shows the difference between the RF-corrected tagger vertex time and the vertex time calculated from the assumed mass value.

where

$$
\beta_{P I D}^{2}=\frac{p^{2}}{m_{P D G}^{2}+p^{2}} .
$$

The momentum of the track, $p$, and the path length to the TOF, $l_{T O F}$, is known from the event reconstruction but the calculated mass is replaced with the accepted value in the Particle Data Group table in Ref. [2]. The bottom right figure in Fig. 3.4 shows the relation of the $t_{v t x}(T O F-P I D)$ and $t_{v t x}(T O F-S T)$. With three charged tracks, the event is kept if at least one track falls within the vertex time cut $t_{v t x}(T O F-S T)$ yet all three tracks are required have $t_{v t x}(T O F-P I D)$ between -1.0 and $1.0 \mathrm{~ns}$ to ensure the track is correctly identified as a $\pi$ or $p$. Fig. 3.5 shows the track momentum versus $\beta$ before and after the timing cuts. The pronounced curves, 


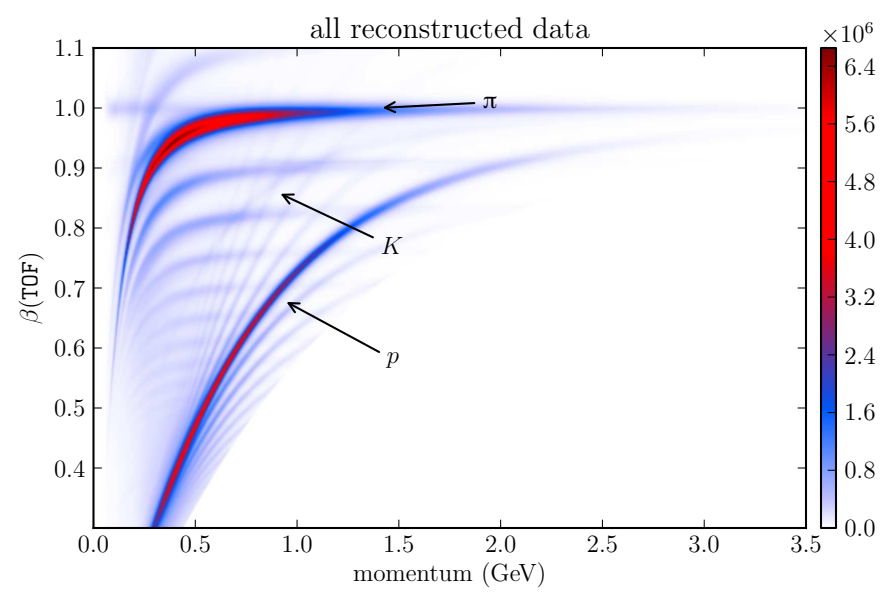

(a) Before timing cuts.

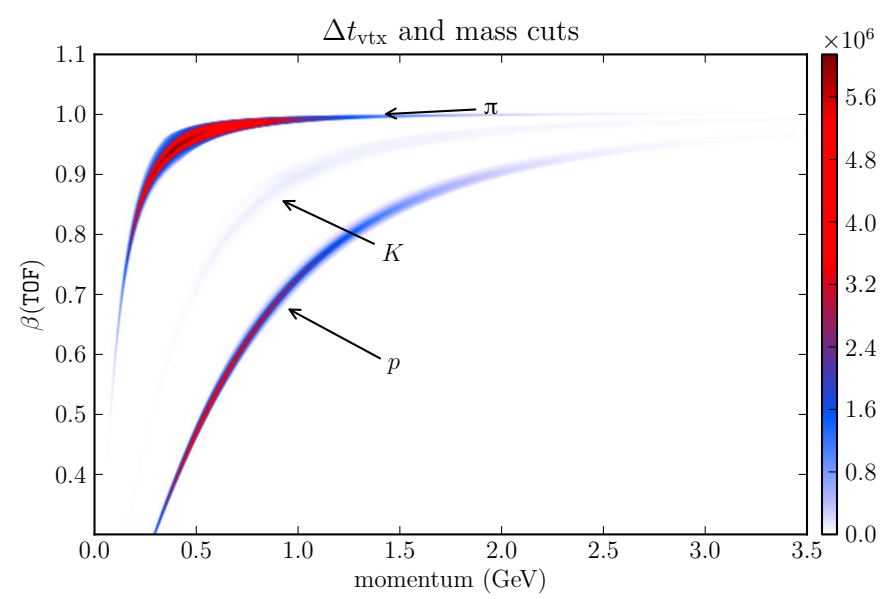

(b) After all timing and PID cuts.

Figure 3.5: The $\beta$ of the track compared to the momentum before and after the timing and particle ID cuts.

Fig. 3.5(a), are for the $\pi$ and $p$ tracks with additional bands that follow the trend but are from different beam buckets and result in overlapping particle types.

\subsection{Energy Loss Corrections}

The event reconstruction of a particle track in CLAS starts in region 1 of the DC after the track has gone through the target and start counter. To reconstruct the track back to the event vertex, additional energy loss from the target and start counter are 
included. The standard CLAS ELOSS package provides the required functionality as detailed in Ref. [26]. ELOSS corrects for the energy lost as charged tracks go from the event vertex through the beam pipe, target, and start counter using the Bethe-Bloch equation [2] to relate the material characteristics and path length to energy loss. Fig. 3.6 shows the energy-loss correction versus the momentum for $\pi^{-}, \pi^{+}$, and $p$. The protons have a correction of $0.01 \mathrm{GeV}$ at high momentum and up to $0.08 \mathrm{GeV}$ for low momentum. The pions have a correction of less than $0.005 \mathrm{GeV}$. 


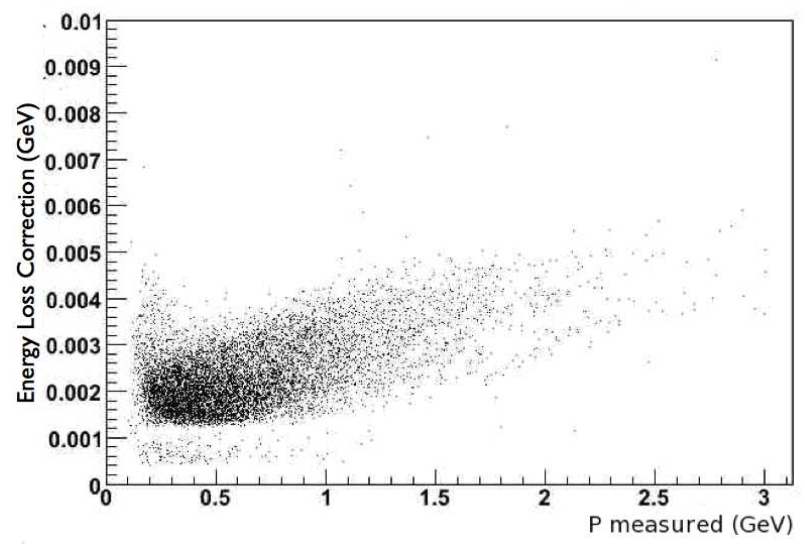

(a) ELOSS correction on $\pi^{-}$tracks.

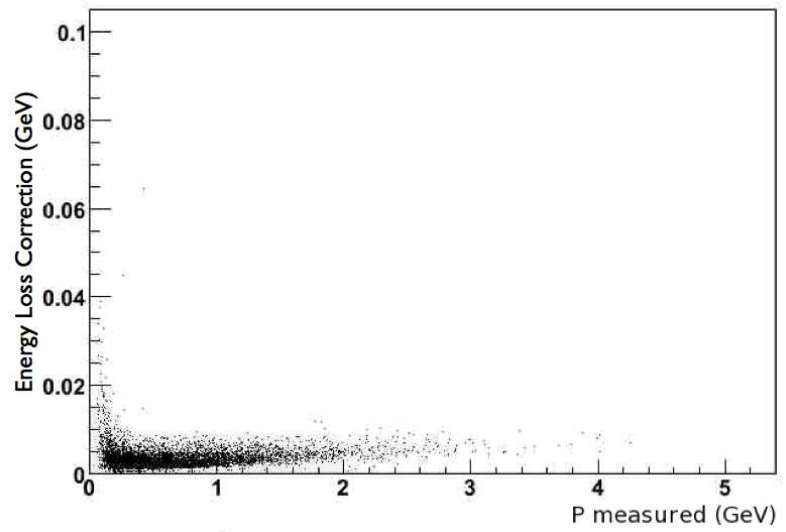

(b) ELOSS correction on $\pi^{+}$tracks.

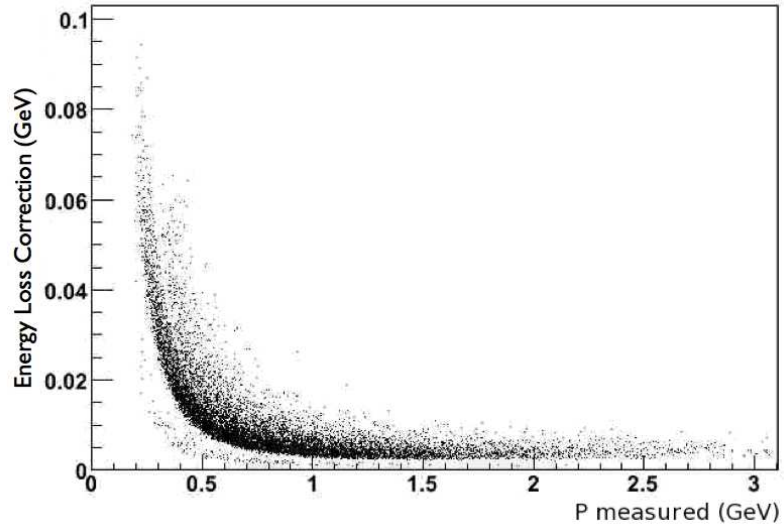

(c) ELOSS correction on $p$ tracks.

Figure 3.6: The energy loss corrections applied using ELOSS. The $y$-axis is the absolute value of the difference between the reconstructed momentum and the corrected momentum, in units of $\mathrm{GeV}$. The $x$-axis is the total momentum of the tracks. 


\subsection{Missing Momentum Cuts}

The channel being investigated in the following analysis is

$$
\gamma p \rightarrow p \pi^{+} \pi^{-} \eta
$$

where the $\eta$ is the neutral particle. A possible source of background for this channel is

$$
\gamma p \rightarrow p \pi^{+} \pi^{-}
$$

To minimize the reaction $\gamma p \rightarrow p \pi^{+} \pi^{-}$background, the transverse component of the missing four-momentum calculated from Eq. 3.4, shown in Fig. 3.7, is required to be greater than $0.085 \mathrm{GeV}$. This cuts out the $p \pi^{+} \pi^{-}$exclusive events at small missing $z$-momentum along with misidentified beam photons at large missing $z$-momentum. The mass squared of the missing particle from $\gamma p \rightarrow p \pi^{+} \pi^{-}(\eta)$ is in Fig. 3.8 and shows clear $\pi^{0}, \eta$, and $\omega$ peaks. 


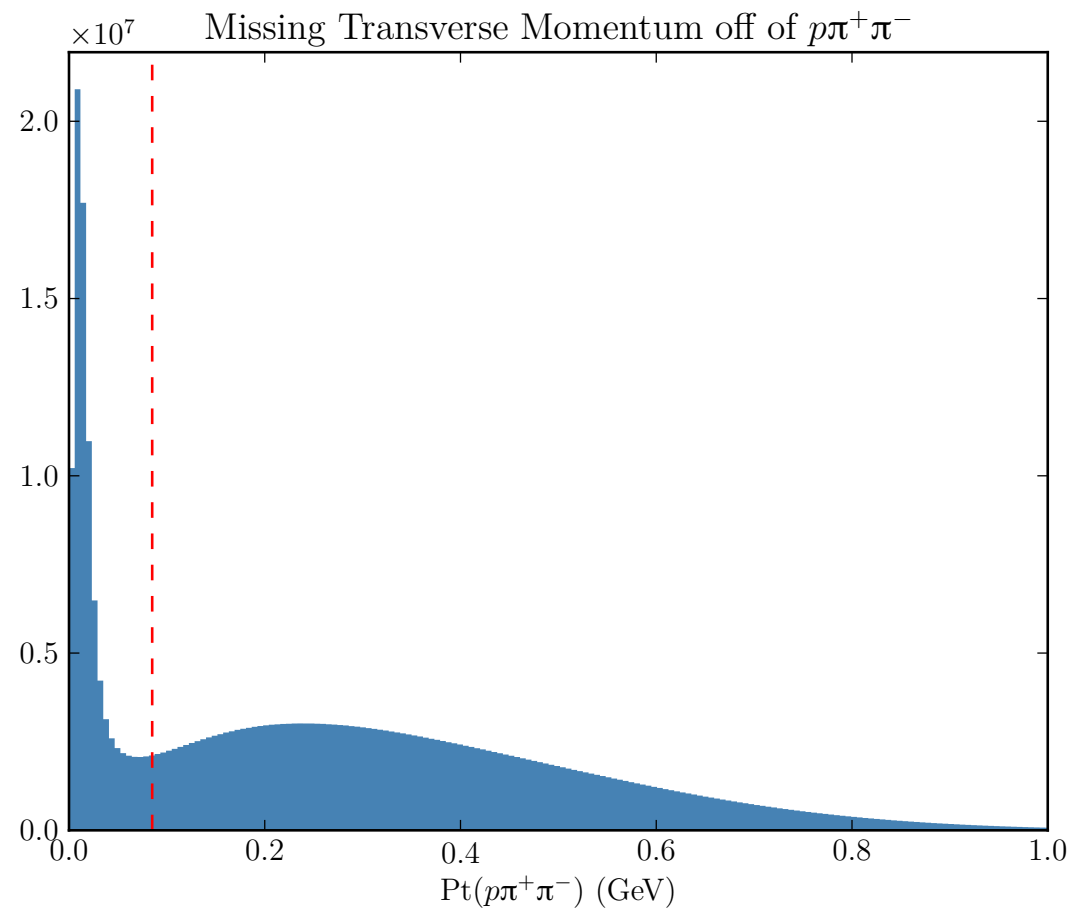

Figure 3.7: Plot of the missing transverse momentum off the $p \pi^{+} \pi^{-}$. The low momentum peak is from $p \pi^{+} \pi^{-}$exclusive events. 


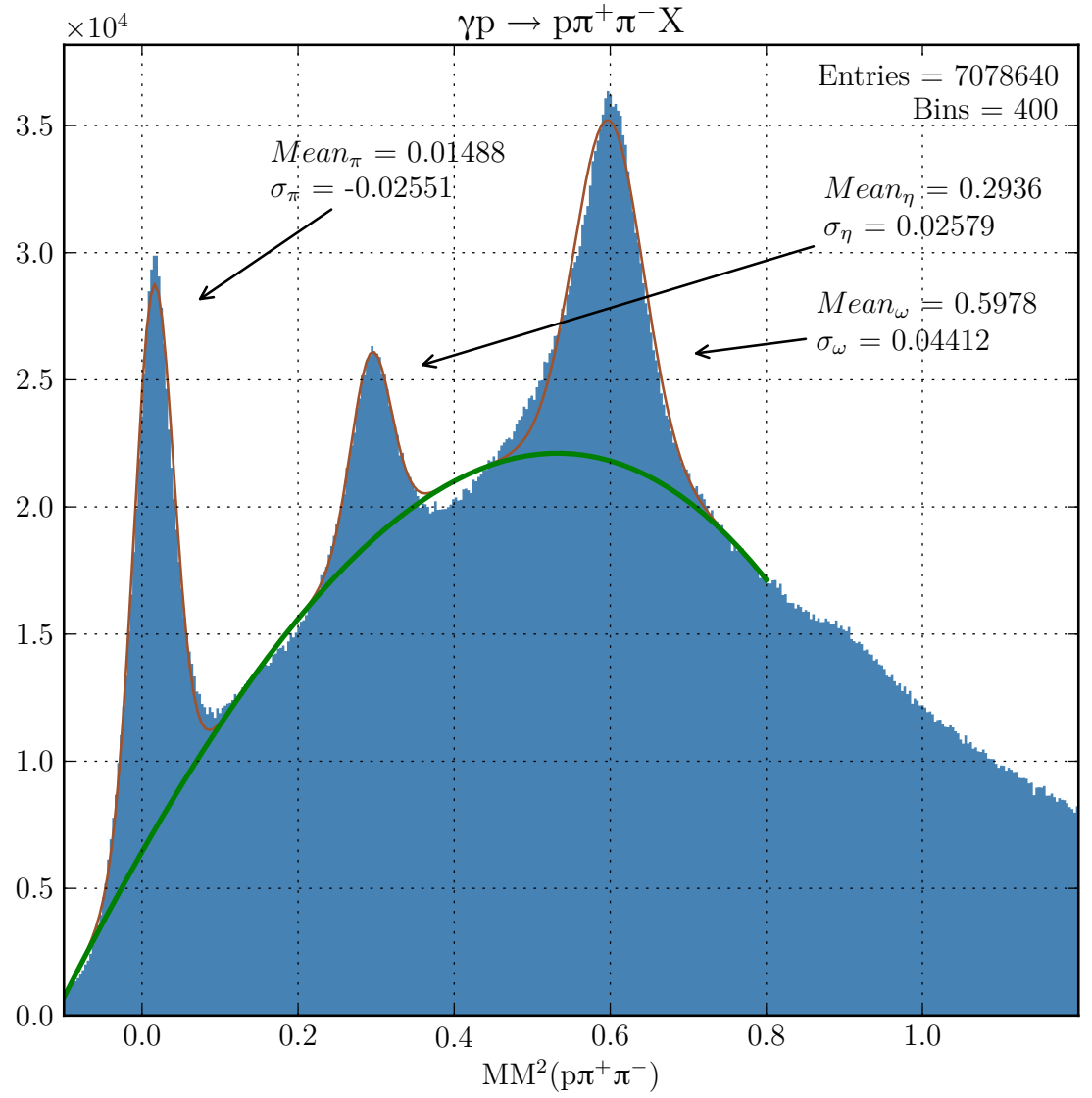

Figure 3.8: Plot of the missing mass squared off of the $p \pi^{+} \pi^{-}$before kinematic fitting of charged tracks. The red curves correspond to Gaussian fits to the peaks and the green curve is a polynomial background. 


\subsection{Additional Missing Mass and $\gamma \gamma$ Invariant Mass Cuts}

The result of the cuts up to this point are shown in Fig. 3.8. The spectrum shows a peak at the mass of the $\eta$ sitting on a very large background. The background includes events where the $p \pi^{+} \pi^{-} \gamma$ were all detected but the events are from a higher particle multiplicity reaction such as $p \pi^{+} \pi^{-}\left(\pi^{+}\right)\left(\pi^{-}\right)\left(\pi^{0}\right)$ or $p \pi^{+} \pi^{-}\left(\pi^{0}\right)\left(\pi^{0}\right)$.

For every decay photon detected, the acceptance is decreased by up to $90 \%$. Although not ideal, the second photon is required to clean up the background. The photon cuts used in this analysis limit the momentum vector of the missing particle to be in the same direction as the four-vector of the $\gamma \gamma$ system. A cut is placed on the absolute value of the total missing mass squared off of $p \pi^{+} \pi^{-} \gamma \gamma$ to be less than $0.02 \mathrm{GeV}^{2}$, as shown in Fig. 3.9. The polar angle difference is

$$
\Delta \theta=\theta_{\gamma \gamma}-\theta_{M M}
$$

where $\theta_{\gamma \gamma}$ is the polar angle reconstructed from the two detected photons and $\theta_{M M}$ is the polar angle reconstructed from the missing momentum vector. $\Delta \theta$ is within 0.05 radians and the difference in $\phi$ is within 0.25 radians, as shown in Figs. 3.10 and 3.11. The missing mass spectrum after these cuts is illustrated in Fig. 3.12 with a $4 \sigma$ cut around the $\eta$ peak. The following section will select the most probable $\eta$ events from the broad missing mass cut using the kinematic fitting technique. 


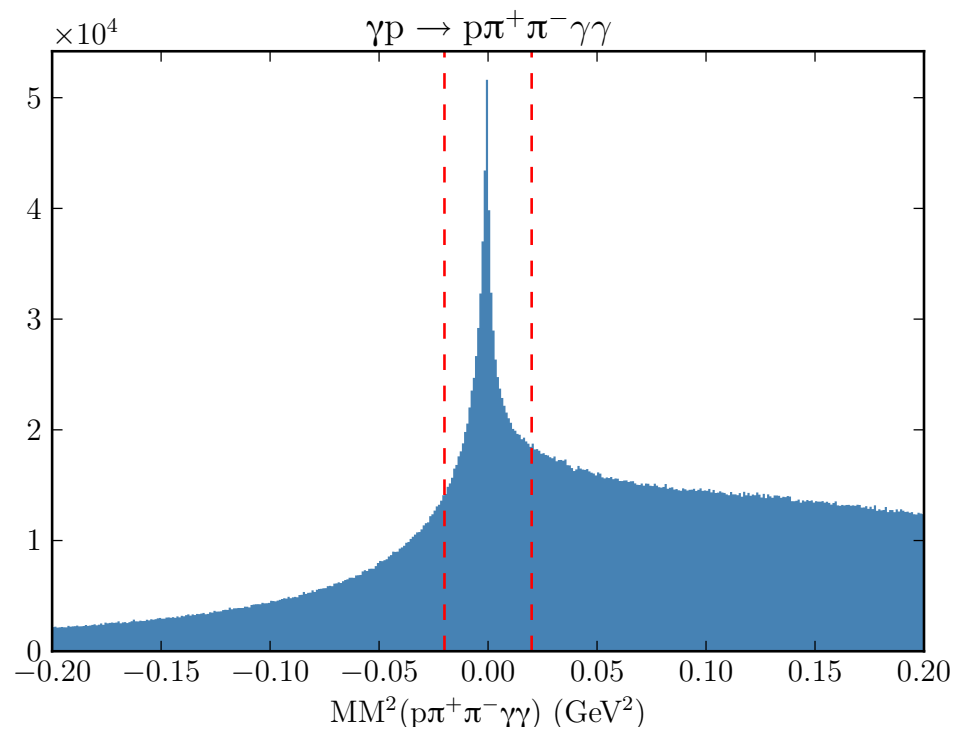

Figure 3.9: Missing mass squared off of $p \pi^{+} \pi^{-} \gamma \gamma$. The dashed red line shows the cut used on the data.

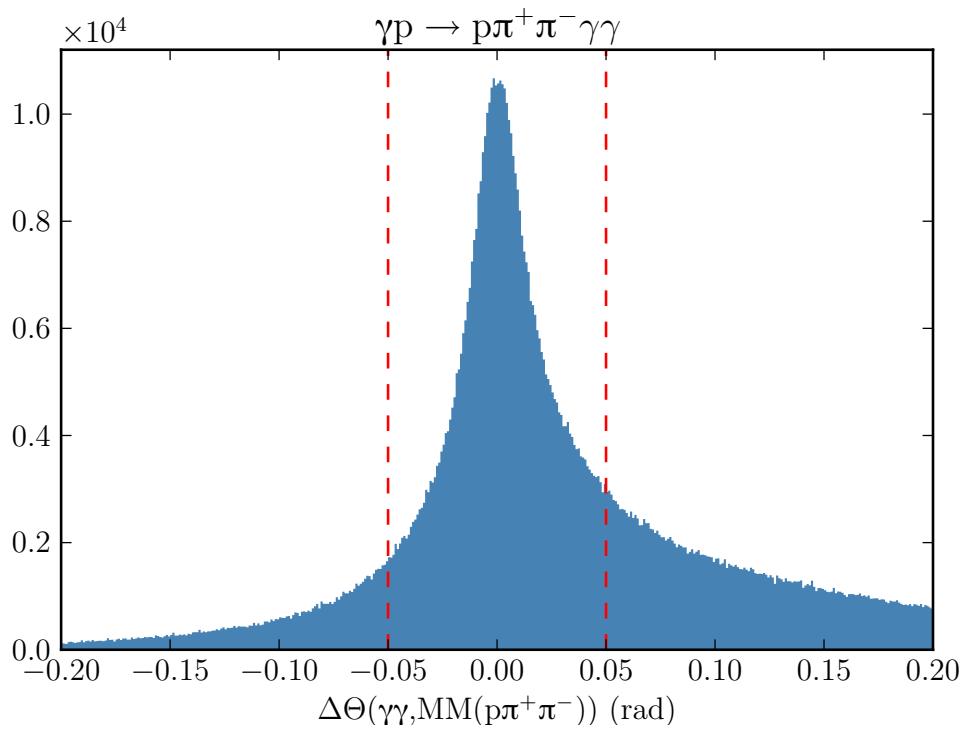

Figure 3.10: The calculated difference between $\theta$ of the $M M\left(p \pi^{+} \pi^{-}\right)$and $\gamma \gamma$. The dashed red line shows the cut used on the data. 


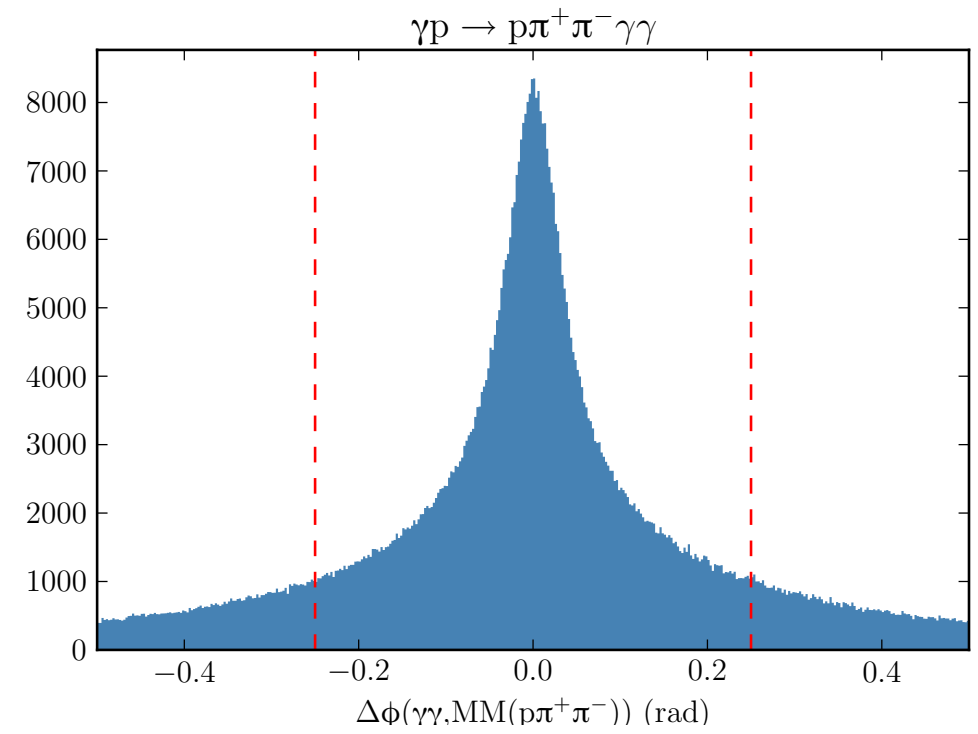

Figure 3.11: The calculated difference between $\phi$ of the $M M\left(p \pi^{+} \pi^{-}\right)$and $\gamma \gamma$ detected. The dashed red line shows the cut used on the data.

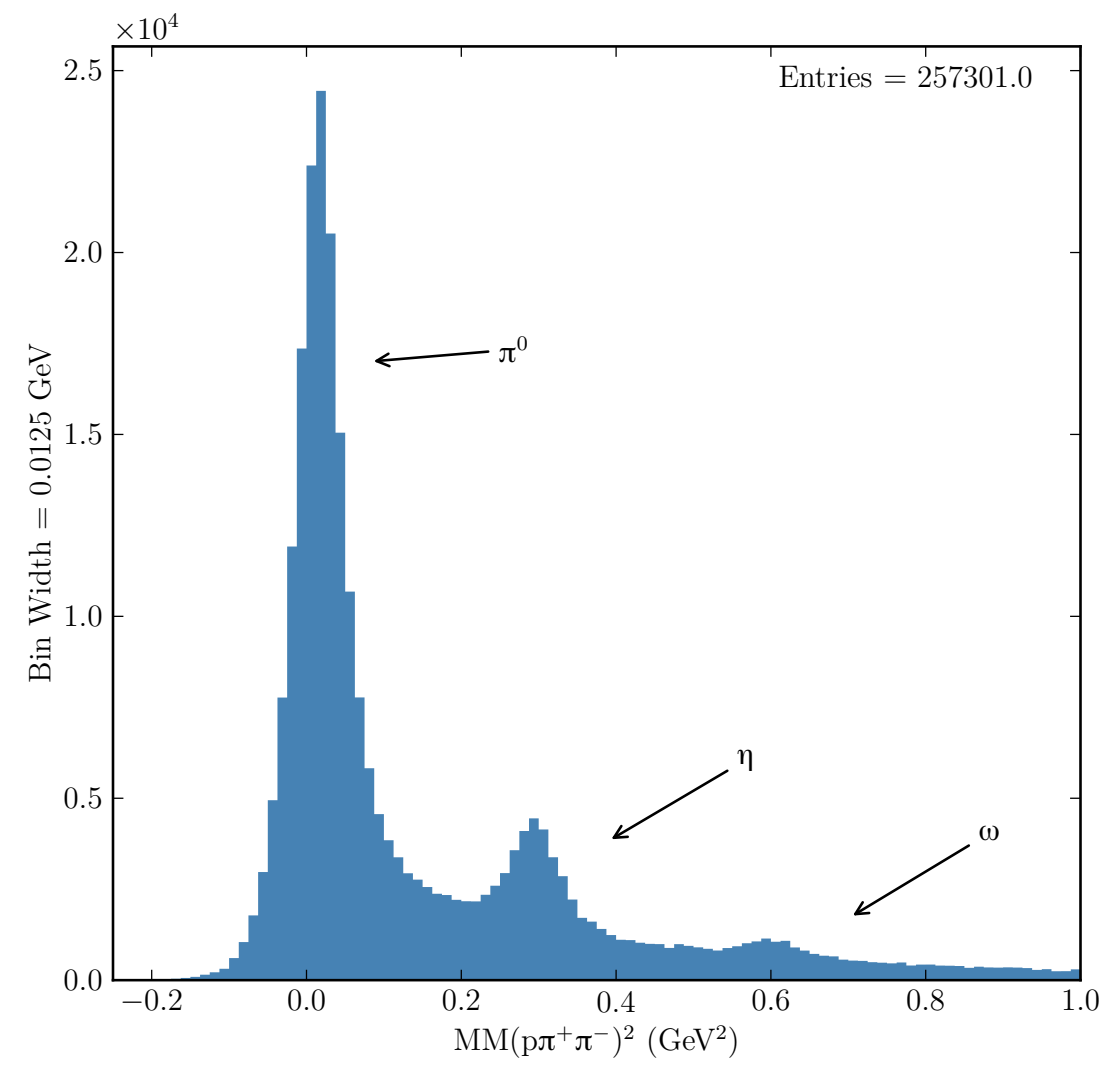

Figure 3.12: Missing mass squared off of $p \pi^{+} \pi^{-}$with the $\gamma \gamma$ detected. 


\subsection{Kinematic Fitting}

Kinematic fitting uses known constraints such as conservation of momentum, conservation energy, and vertex position to improve measured quantities. It is expressed as

$$
\overrightarrow{\eta_{i}}=\overrightarrow{\eta_{f}}+\vec{\epsilon}
$$

where $\overrightarrow{\eta_{i}}$ is the set of measured observables, $\overrightarrow{\eta_{f}}$ is the set of true values, and $\vec{\epsilon}$ is the set of deviations needed to shift the observed values. During track reconstruction, a covariance matrix is calculated taking into account resolution uncertainties and then tracking parameters for each track are correlated.

There are additional energy loss, and multiple scattering corrections made to these matrices before feeding them into a kinematic fitter. Further detail on the algorithm to adjust the covariance matrix is explained in Ref. [27]. The kinematic fitter used in this analysis was written and developed by Dustin Keller and is based on the method of Lagrange multipliers to handle the constraints with a least squares method. The quality of the fit is assessed by examining the pull distributions, where a pull is defined as the difference between the measured and final parameters obtained by the kinematic fit and normalized by the quadratic error difference. The pull for the initial value, $\eta_{i}$, and final value, $\eta_{f}$, of the measured quantities is

$$
z=\frac{\eta_{i}-\eta_{f}}{\sqrt{\sigma_{\eta_{i}}^{2}-\sigma_{\eta_{f}}^{2}}}
$$

where $\sigma_{\eta_{i}}^{2}$ and $\sigma_{\eta_{f}}^{2}$ are the corresponding covariance matrix elements for $\eta_{i}$ and $\eta_{f}$. If the input covariance matrix is correctly adjusted, the pulls will be normally distributed with a zero mean and have a unit standard deviation. The covariance matrix was tuned on $p \pi^{+} \pi^{-}\left(\pi^{0}\right)$ where the detected $\gamma \gamma$ invariant mass is $\pi^{0}$ is used because of the channel's high signal-to-background ratio. The results of the tuning on $p \pi^{+} \pi^{-}\left(\pi^{0}\right)$ is in Fig. 3.13. 

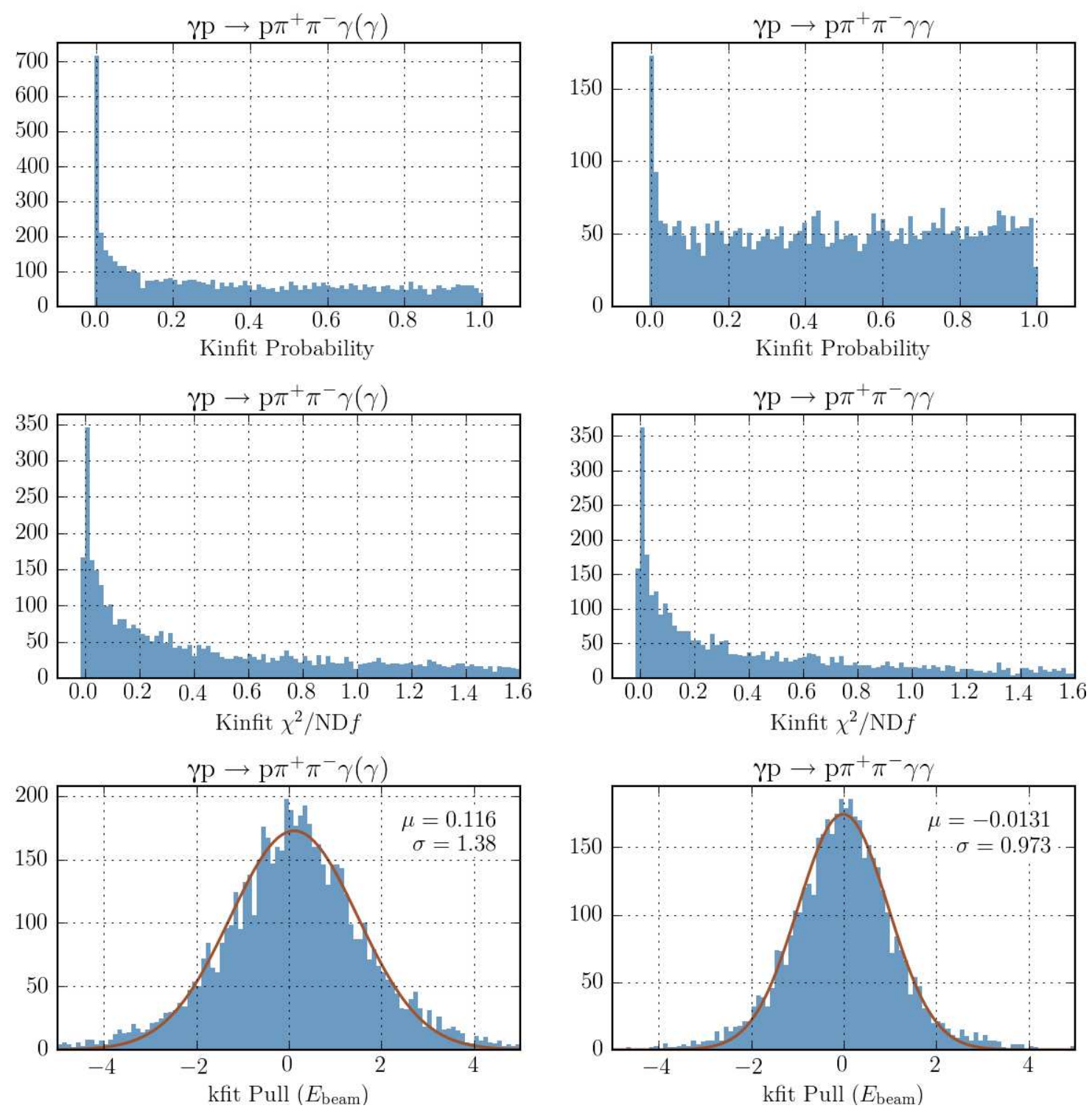

Figure 3.13: Kinematic fit results of $p \pi^{+} \pi^{-}\left(\pi^{0}\right)$ where one and two $\gamma^{\prime}$ 's were detected. The left plots are for events with $p \pi^{+} \pi^{-} \gamma(\gamma)$ and the right are events with $p \pi^{+} \pi^{-} \gamma \gamma$. Top plots are the probability from the fit and show the distribution to be flat with a peak at 0.0. The middle plots are the $\chi^{2}$ of the kinematic fits. The lower plots are the pulls of the fit and are related to the energy and momenta corrections. They are centered at 0.0 and have a $\sigma$ close to 1.0 . 
The "goodness" of the fit is quantified by the confidence level of the fit, CL, which is the integral of the $\chi^{2}$ probability density function, $f(z, n)$, for $n$ degrees of freedom;

$$
C L=\int_{\chi^{2}}^{\infty} f(z, n) d z .
$$

The confidence level indicates the probability that a chosen event would have a $\chi^{2}$ greater than the result of the fit. Events described by the hypothesis will result in a uniform distribution from 0 to 1 , and those poorly described will result in a sharp peak at zero. A minimum CL cut is used to select the events corresponding to the physics hypothesis.

The final results for $p \pi^{+} \pi^{-}(\eta)$ are shown in Fig. 3.15 for exclusive skim $p \pi^{+} \pi^{-} \gamma \gamma$. The confidence level of the fit was required to be at least $1 \%$ to select $\eta$ from the neutral mass in Fig. 3.12. 


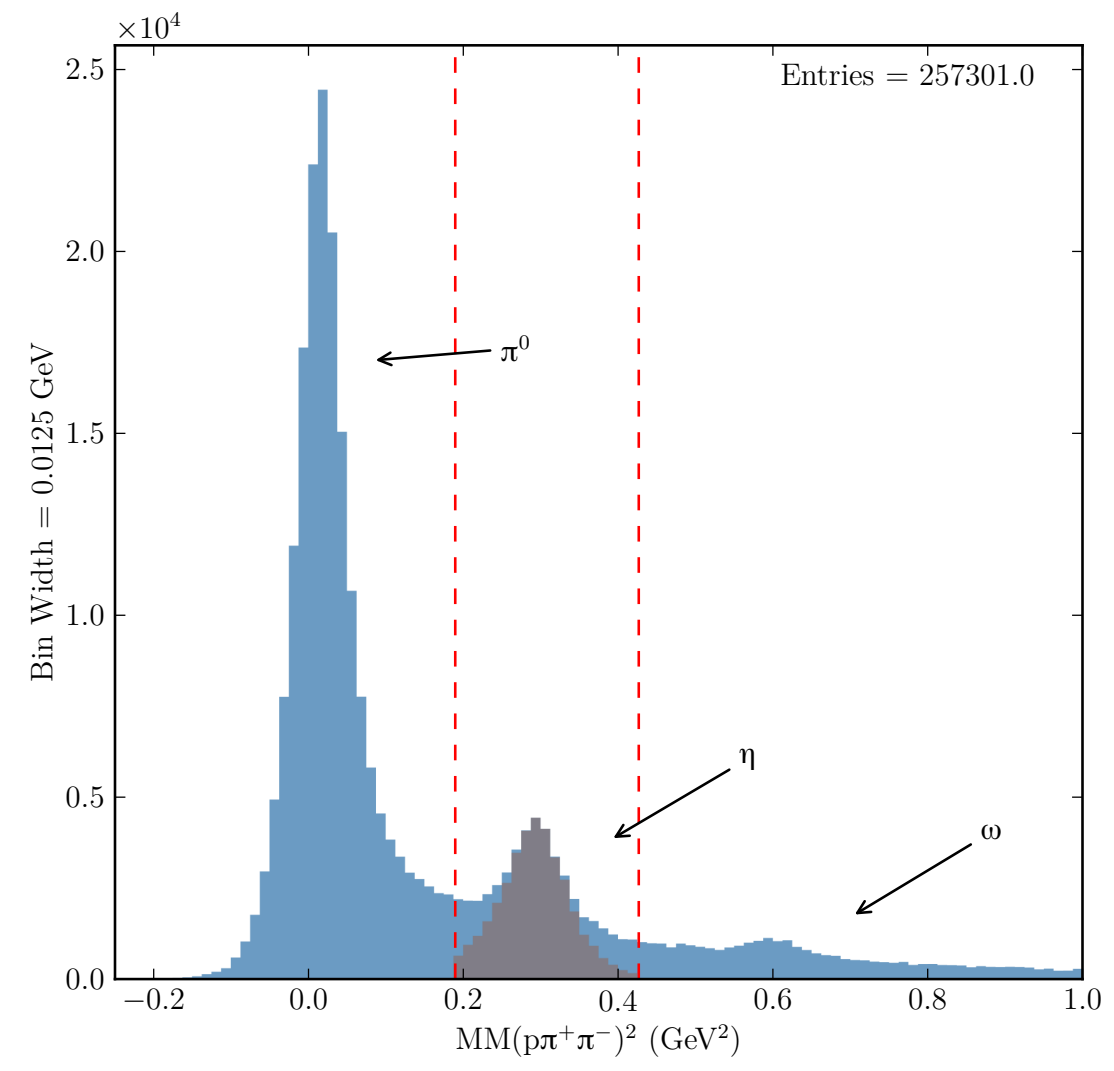

Figure 3.14: Missing mass squared off of $p \pi^{+} \pi^{-}$, with the red line to indicate the cut used for selecting the $\eta$ to be kinematically fit. The brown distribution contains events that passed the CL cut. 


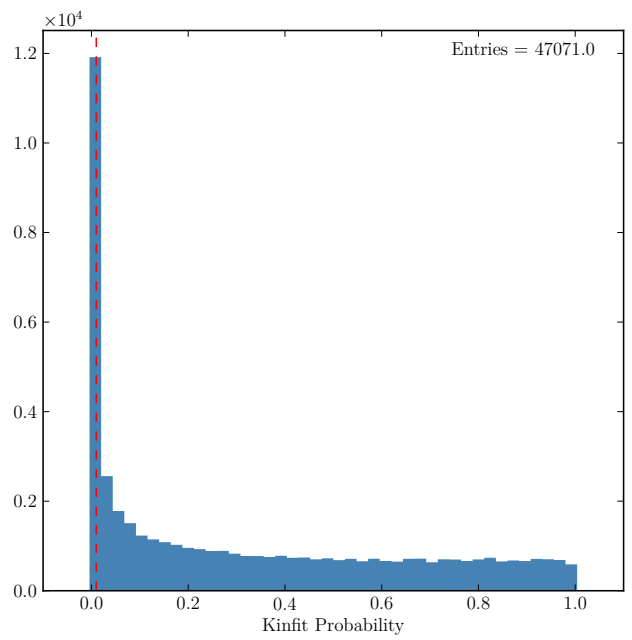

(a) Final Confidence Level.

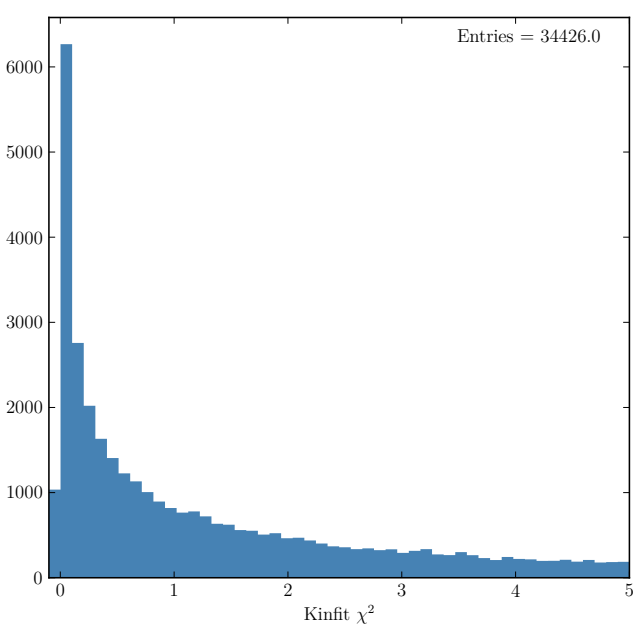

(b) Final $\chi^{2}$.

Figure 3.15: The final results of the kinematic fit of $p \pi^{+} \pi^{-}(\eta)$. Plot (a) shows that the CL distribution is flat with a peak at 0.0. Events with low probabilities of being from $p \pi^{+} \pi^{-}(\eta)$ have a CL close to 0.0. Plot (b) shows the $\chi^{2}$ where the expected distribution peaks to the right of 0.0 and has a decline as $\chi^{2}$ increases. The red line indicates the CL cut used in this analysis.

\subsection{Final Event Selection}

The cuts discussed above have included only those needed for basic particle identification. The resulting data set includes the following topologies:

$$
\begin{aligned}
\gamma p & \rightarrow \Delta^{++} X^{-}, \\
& \rightarrow \Delta^{0} X^{+} \\
& \rightarrow p \eta(1295) \rightarrow p a_{0} \pi \\
& \rightarrow p f_{1}(1285) \rightarrow p a_{0} \pi \\
& \rightarrow \rho p \eta, \\
& \rightarrow \Delta^{*++} \pi^{-} \rightarrow \Delta^{++} \eta \pi^{-}
\end{aligned}
$$

A partial wave analysis is capable of yielding the wave contribution for a resonance $X$ even for situations with few statistics, however reactions 3.16 to 3.20 interfere with 
reaction 3.15 in a non-trivial way that cannot be handled by the isobar model PWA method used in this analysis. The following set of cuts is used to isolate the $\Delta^{++} X^{-}$ contributions and are discussed in the following sections and include

- $\Delta^{++} \rightarrow p \pi^{+}$

$$
-M\left(p \pi^{+}\right)<1.3 \mathrm{GeV}
$$

- $\eta \rightarrow \gamma \gamma$

$$
-|M(\gamma \gamma)-0.55|<0.1 \mathrm{GeV}
$$

- Momentum Transfer

$$
-|t|<0.5 \mathrm{GeV}^{2}
$$

\subsection{1 $\Delta^{++} \rightarrow p \pi^{+}$Selection}

The $p \pi^{+}$invariant mass distribution in Fig. 3.16 shows a $\Delta(1230)$ peak followed by a small peak near the $N(1440)$ mass. The $\Delta^{++}$is selected by requiring the invariant mass of the $p \pi^{+}$to be less than $1.3 \mathrm{GeV}$. Processes with the same final-state topology that may interfere with this signal include: $\gamma p \rightarrow \Delta^{0} \eta \pi^{+}$and $\gamma p \rightarrow N^{* 0} \eta \pi^{+}$. After placing the cut on the $p \pi^{+}$invariant mass, the invariant mass of the $p \pi^{-}$system shows no signs of $\Delta^{0}$ or $N^{*}$ peaks effectively removing the $\Delta^{0} X^{+}$topology as seen in Fig. 3.17.

\subsection{2 $\eta \rightarrow \gamma \gamma$ Selection}

The invariant mass of $\gamma \gamma$ is calculated from the energy deposited in the EC:

$$
M(\gamma \gamma)=\sqrt{2 E_{\gamma_{1}} E_{\gamma_{2}} *\left(1-\cos \theta_{\gamma \gamma}\right)}
$$




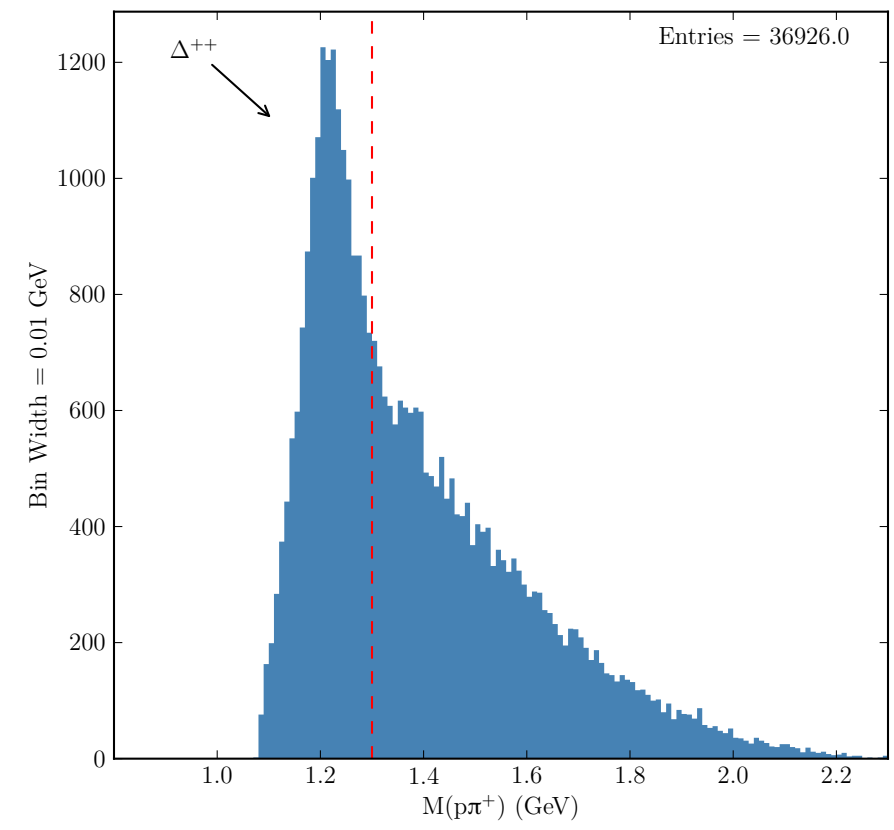

Figure 3.16: Invariant mass of $p \pi^{+}$after kinematic fit for $p \pi^{+} \pi^{-}(\eta)$ and the CL cut.

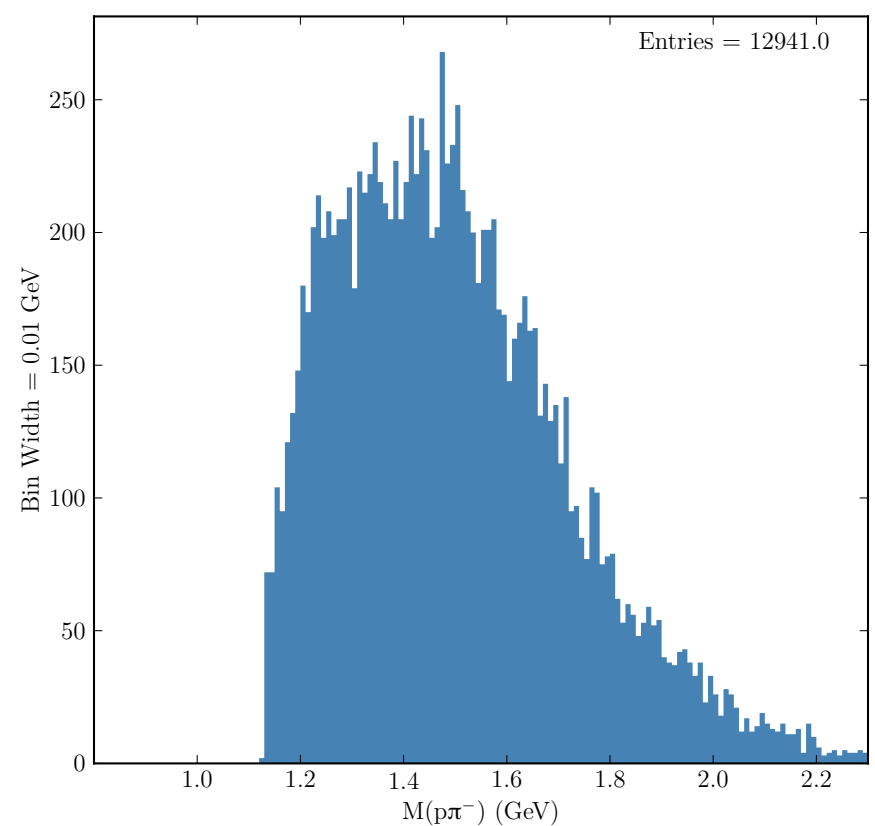

Figure 3.17: Invariant mass of $p \pi^{-}$after the $\Delta^{++}$invariant mass selection. 
where $\theta_{\gamma \gamma}$ is the opening angle between the two photons decaying from either a $\eta$ or $\pi^{0}$. The mass resolution of the photon pairs from the $\eta$ decay is dominated by the uncertainties in the energy measured in the EC and results in a broadening of the $\eta$ peak as shown in Fig. 3.18. The two-photon decay of an $\eta$ is selected taking the $\gamma \gamma$ invariant mass to be $0.55 \pm 0.1 \mathrm{GeV}$ as shown in Fig. 3.18.

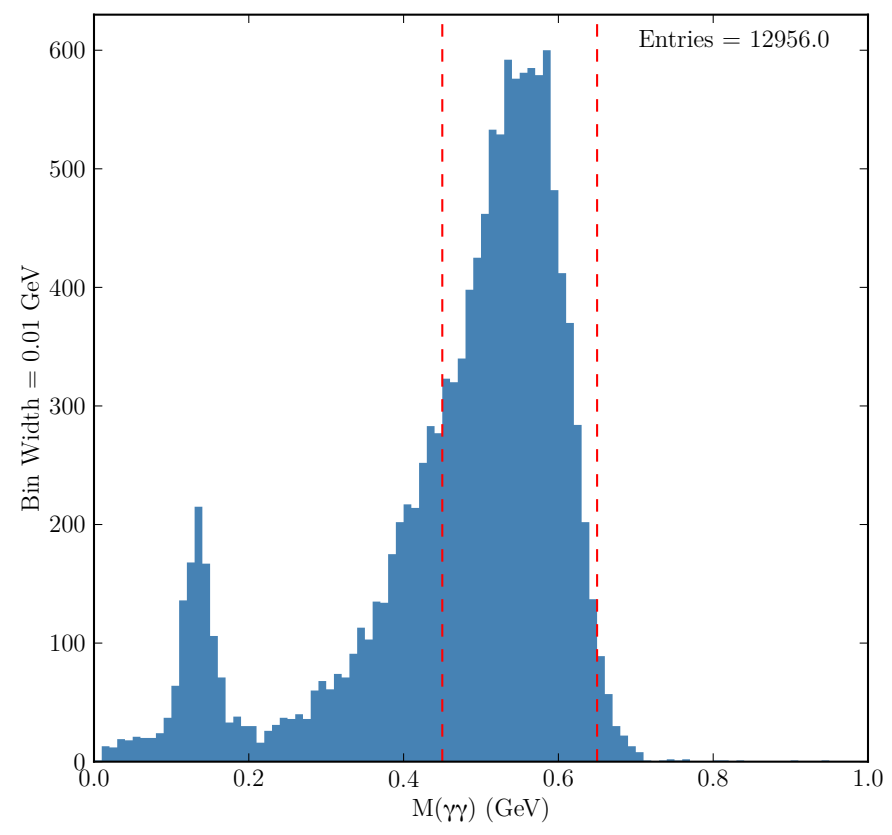

Figure 3.18: Plot of the invariant mass of $\gamma \gamma$ after the CL cut (see Sec. 3.6). The dashed lines indicate the cut on the $\eta$ mass.

\subsubsection{Momentum Transfer Selection}

As shown in Fig. 3.19, the invariant mass of $\eta \pi^{-}$contains the $a_{0}$ and $a_{2}$ resonances, which will be included in the PWA. The location of the exotic resonance of interest is located at $1.4 \mathrm{GeV}$. The momentum transfer is defined by

$$
t=\left(P_{\text {beam }}-P_{\eta \pi^{-}}\right)^{2}
$$




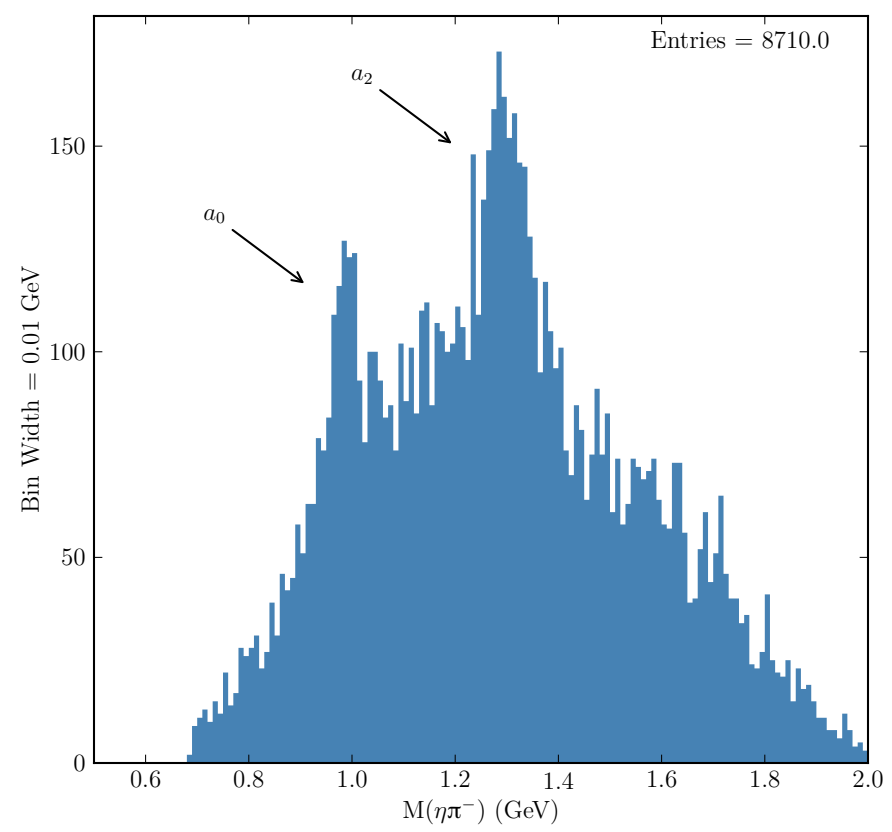

Figure 3.19: Invariant mass of $\eta \pi^{-}$. The $a_{0}(980)$ and $a_{2}(1320)$ peaks in the invariant mass of $\eta \pi^{-}$.

and is expected to follow $A e^{-b t}$ for a peripheral meson production [28]. Fig. 3.21 shows the $t$ distributions for the events under the $a_{2}, a_{0}$ peaks and in the background region, which is above the expected peak of the $\pi_{1}(1400)$. The $a_{0}$ and background dominate the $t$ distribution below $-0.5 \mathrm{GeV}^{2}$ leading to a cut of $|t|<0.5 \mathrm{GeV}^{2}$ to remove the background from the non- $\eta \pi^{-}$resonances. The cut on momentum transfer results in Fig. 3.22 where the $\eta \pi^{-}$invariant mass shows a peak for the $a_{2}$ with a reduction in the background and the $a_{0}$ peak.

The amplitudes used in the partial wave analysis are a function of $t$, beam energy, resonance mass, and angle of the decay products. For this analysis, because of limited statistics, the amplitude is averaged over the energy range $E_{\gamma} \in[4.45,5.45]$. The full decay amplitudes have a $t$ dependence that introduces a strong model dependence to the PWA. For experimental analysis it is appropriate to bin in $t$ and treat the ampli- 


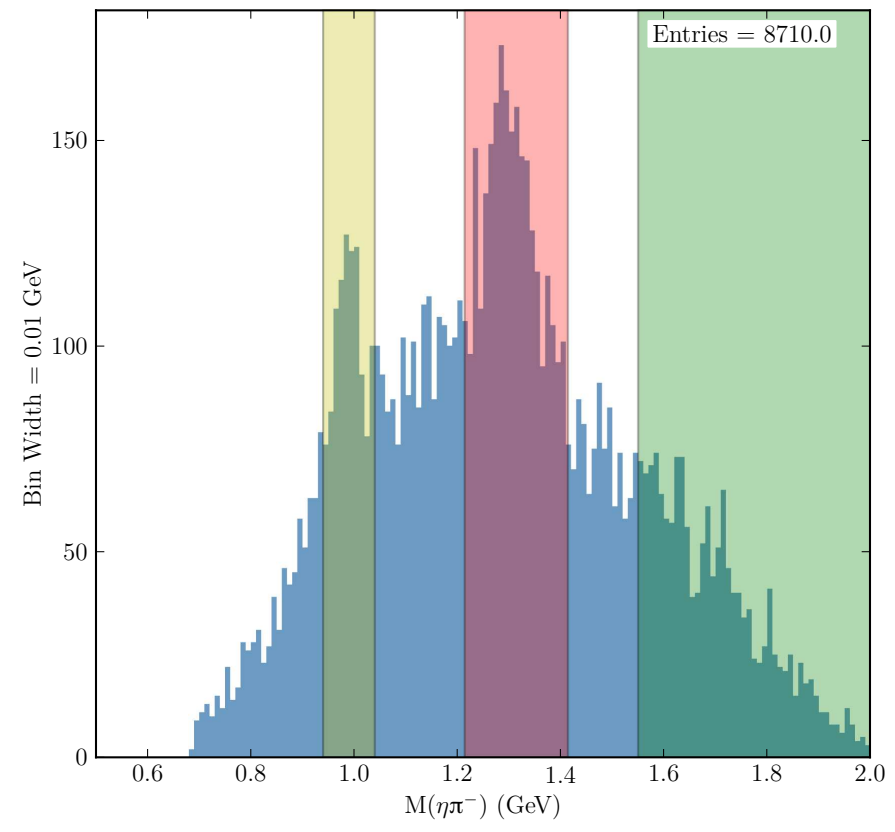

Figure 3.20: Invariant mass of $\eta \pi^{-}$, with mass regions highlighted. The $a_{0}(980)$ region is highlighted in yellow, $a_{2}(1320)$ is highlighted in red, and the high mass region is highlighted in green. 


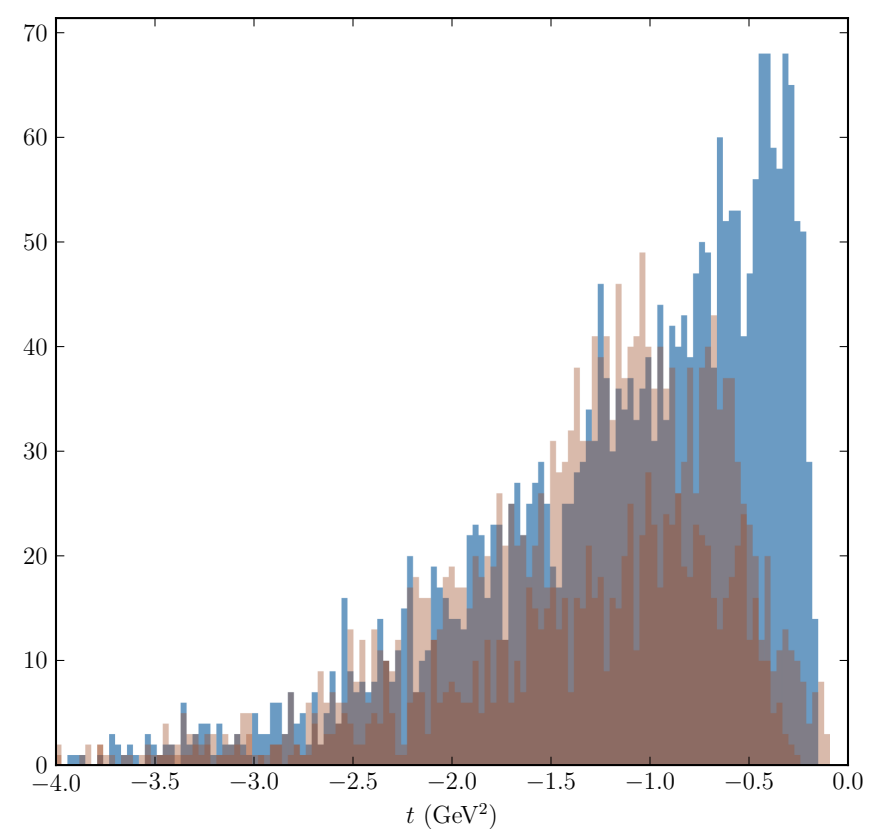

Figure 3.21: The momentum transfer, $t$, of the events under the $a_{2}(1320)$ peak. The $a_{2}(1320)$ peak is in blue (red region in Fig. 3.20), the events in light brown are the background events to the right of the $a_{2}$ peak (green region in Fig. 3.20), and the darker brown is the events under the $a_{0}$ peak (yellow region in Fig. 3.20). 


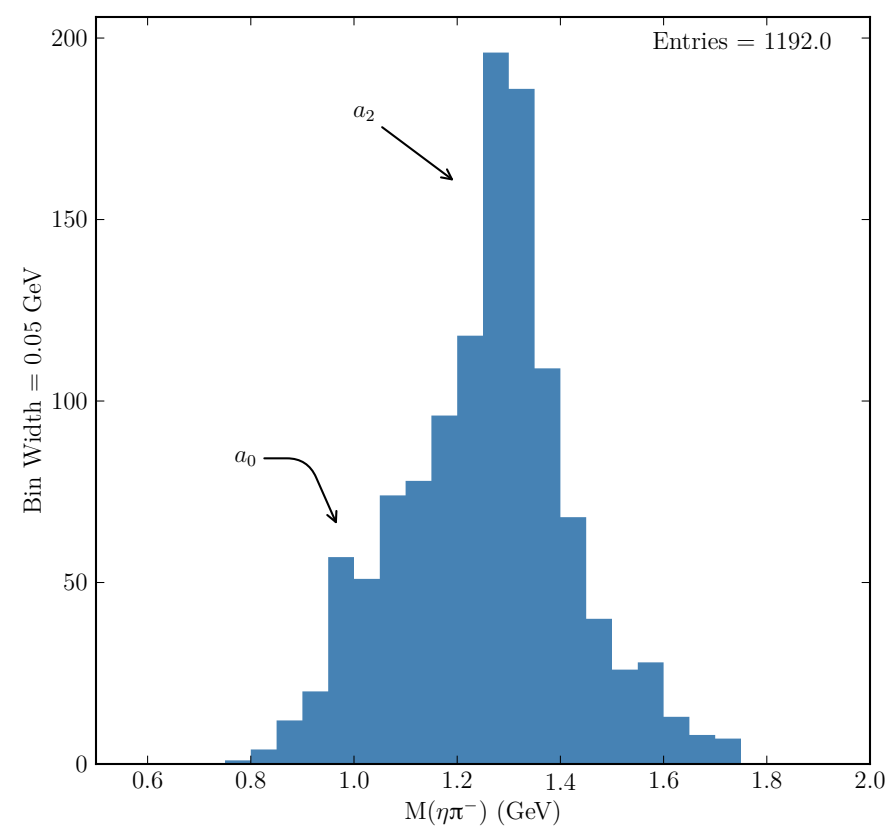

Figure 3.22: Invariant mass of $\eta \pi^{-}$after $t$ cut. The predominant peak remaining is the $a_{2}$ with reduced contribution from the $a_{0}$.

tudes as constant over each bin. With the $t$ selection already made and the limited statistics, the partial wave analysis includes one $t$ bin. The final decay amplitudes are calculated as functions of the decay angles and are binned in terms of the resonance mass.

\subsubsection{Possible Interfering Processes to $\pi_{1}(1400)$ Production}

The invariant mass of $\eta \pi^{+}$for the events used in the PWA are shown in Fig. 3.23. There is no pronounced peak for the $a_{2}$, but a broad peak for the $a_{0}^{+}(980)$ can be seen, which is the result of a combination of acceptance and re-scattering effects.

A possible interfering reaction: $\gamma p \rightarrow \rho N^{*}$, is a result of an $\eta$ exchange particle. The $p \eta$ invariant mass distribution as shown in Fig. 3.24 is out of the range of possible $N^{*}$ with masses less than $1.9 \mathrm{GeV}$. The invariant mass of the $\pi^{+} \pi^{-}$before 


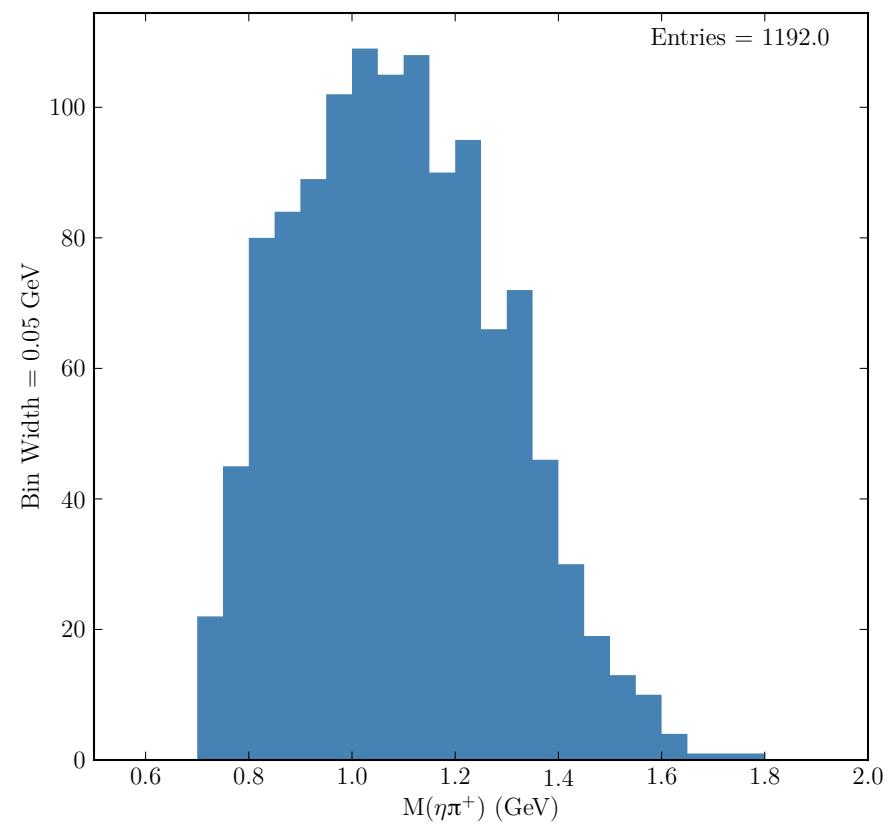

Figure 3.23: Invariant mass distribution of $\eta \pi^{+}$in the low $|t|$ range.

and after the $t$ range selection as shown in Figs. 3.25 and 3.26 does not show a peak corresponding to the $\rho$ mass at $0.775 \mathrm{GeV}$. The highest possible mass of a $\Delta$ or $N^{*}$ with $I<3 / 2$ and $J<3 / 2$ is $1.93 \mathrm{GeV}$ and $1.72 \mathrm{GeV}$, both of which have low statistics and no outstanding peaks in the kinematic range investigated.

Another background to consider is $\gamma p \rightarrow \eta(1295) / f_{1}(1285) p$. The invariant mass of $\eta \pi^{+} \pi^{-}$shown in Fig. 3.27. No peaks corresponding to $f_{1}(1285)$ and $f(1420)$ states are seen.

The final competing reaction considered comes from an intermediate $\Delta^{*++}$ decaying to $\Delta^{++} \eta$. There is a limited number of events in the $p \pi^{+} \eta$ invariant mass spectrum from 1.63 to $2.5 \mathrm{GeV}$ (Fig. 3.28) with no indication of any peaks. Under further study, with the full tagger energy range, there is a peak at $1.93 \mathrm{GeV}$ in the spectrum but it is ignored since it is not present in the energy range selected for this analysis. 


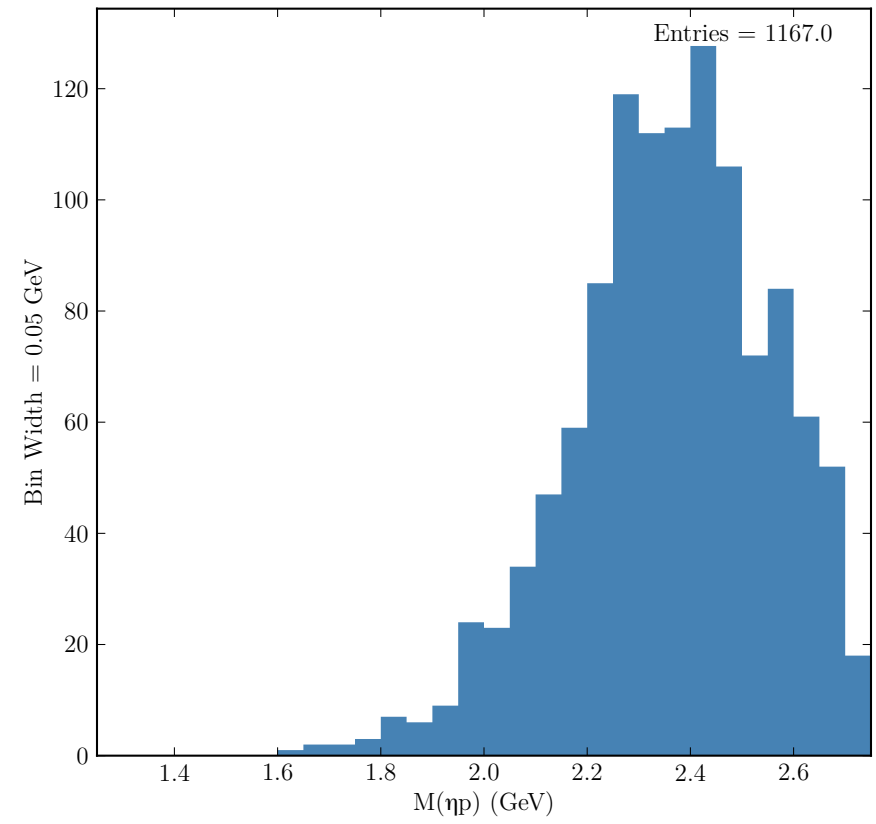

Figure 3.24: Invariant mass distribution of $\eta p$ in the low $|t|$ range.

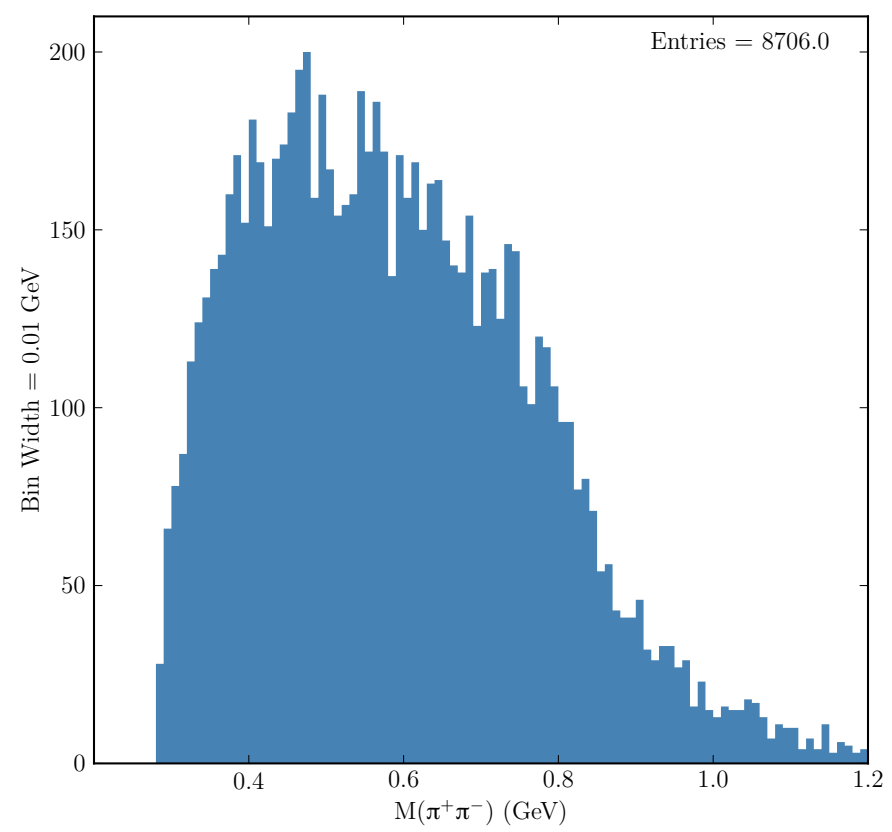

Figure 3.25: Invariant mass distribution of $\pi^{+} \pi^{-}$. 


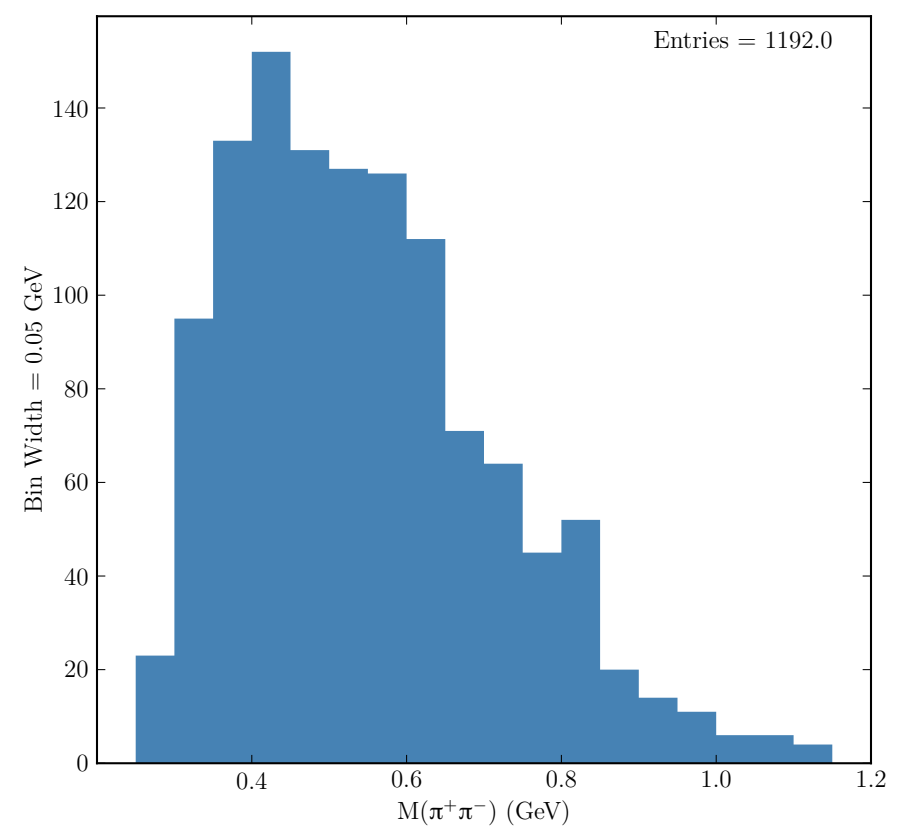

Figure 3.26: Invariant mass distribution of $\pi^{+} \pi^{-}$in the low $|t|$ range.

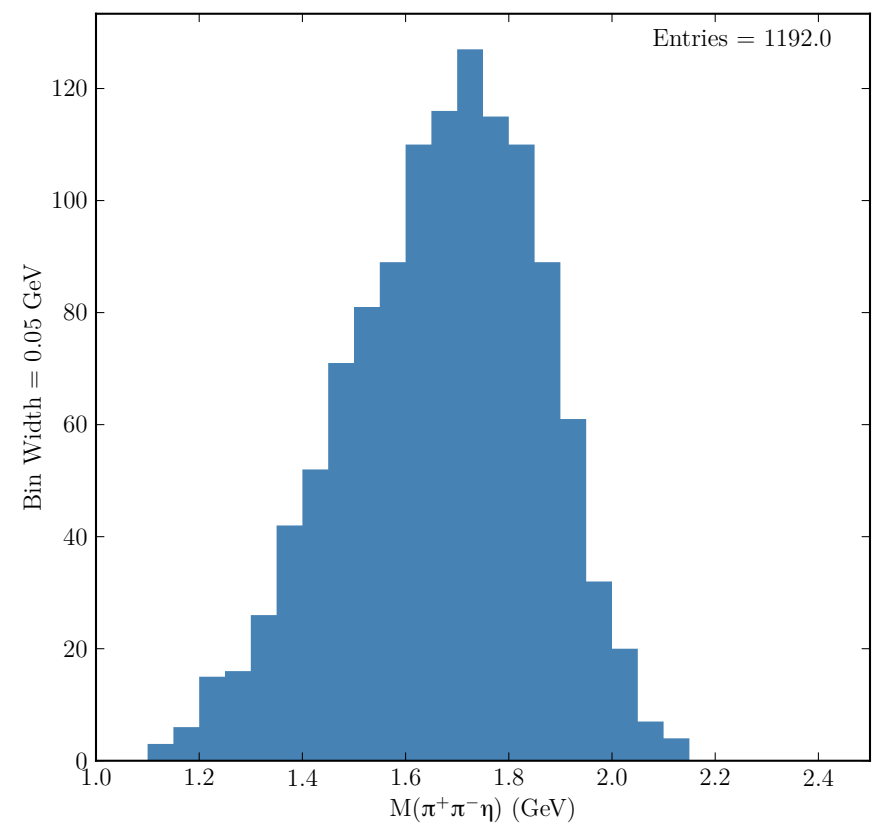

Figure 3.27: Invariant mass distribution of $\eta \pi^{+} \pi^{-}$in the low $|t|$ range. 


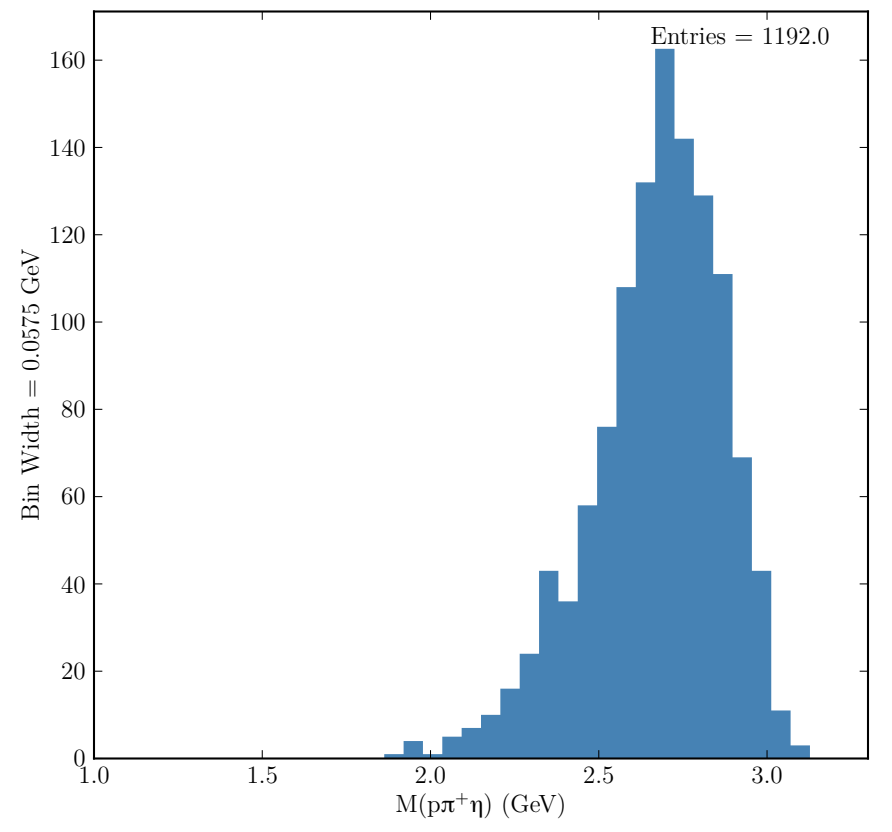

Figure 3.28: Invariant mass distribution of $\eta p \pi^{+}$in the low $|t|$ range.

\begin{tabular}{|c|c|}
\hline cut used & number of events \\
\hline & $2.259 \times 10^{10}$ \\
vtime & $3.342 \times 10^{8}$ \\
$E_{\text {beam }}$ & $8.357 \times 10^{6}$ \\
vertex & $7.078 \times 10^{6}$ \\
$\gamma \gamma$ & $2.573 \times 10^{5}$ \\
$4 \sigma$ on $M M=\eta$ & $4.707 \times 10^{4}$ \\
$\mathrm{CL}$ & $3.693 \times 10^{4}$ \\
$\Delta^{++}$ & $1.283 \times 10^{4}$ \\
$M(\gamma \gamma)$ & 8,722 \\
$t$ & 1,192 \\
\hline
\end{tabular}

Table 3.1: Summary of data cuts used. 


\section{CHAPTER 4}

\section{PARTIAL WAVE ANALYSIS}

Although the peak at $1.3 \mathrm{GeV}$ in the $\eta \pi^{-}$(shown in Fig. 3.22) is consistent with the $a_{2}$ meson. A partial wave analysis (PWA) is used in order to extract quantum numbers. From the known quantum numbers of the $p$ and $\Delta^{++}$, a set of possible states can be established but provide no definitive answers to the exact proportions of the contributions.

A PWA is used to parameterize the amplitude of a spectrum as a function of the angular distributions of the decay products. Measured physical properties such as momentum and energy of the decay products $\left(\eta\right.$ and $\left.\pi^{-}\right)$are used to extrapolate the properties of intermediate states that cannot be measured directly. The decay amplitudes are directly calculated from the data and the production amplitudes are found by fitting the data to find the most probable combinations.

In the Feynman diagram shown in Fig. 4.1, $X$ is described by the two points of intersection. Where the first point of intersection is the intersection of the beam and exchange particle, these form the unknown production amplitude, $V$. The second point is where $\mathrm{X}$ decays to $\eta \pi^{-}$, where the decay products form the decay amplitude, $A$. The intensity of the resonance $X$ is:

$$
I=\left|\sum_{\alpha} V_{\alpha} A_{\alpha}\right|^{2}
$$

where the $V$ is unknown and $A$ can be derived from the measured distributions of the kinematic variables of the $\eta$ and $\pi^{-}$. The sum is over all quantum numbers that describe $X$, where $\alpha$ is the set $\{J, P, M, L, I, \lambda, S\}$. The quantum numbers used to describe the partial waves of resonance $X$ decaying to $\eta \pi^{-}$are

- $J$, total angular momentum of $X$

- $P$, parity of $X$ 


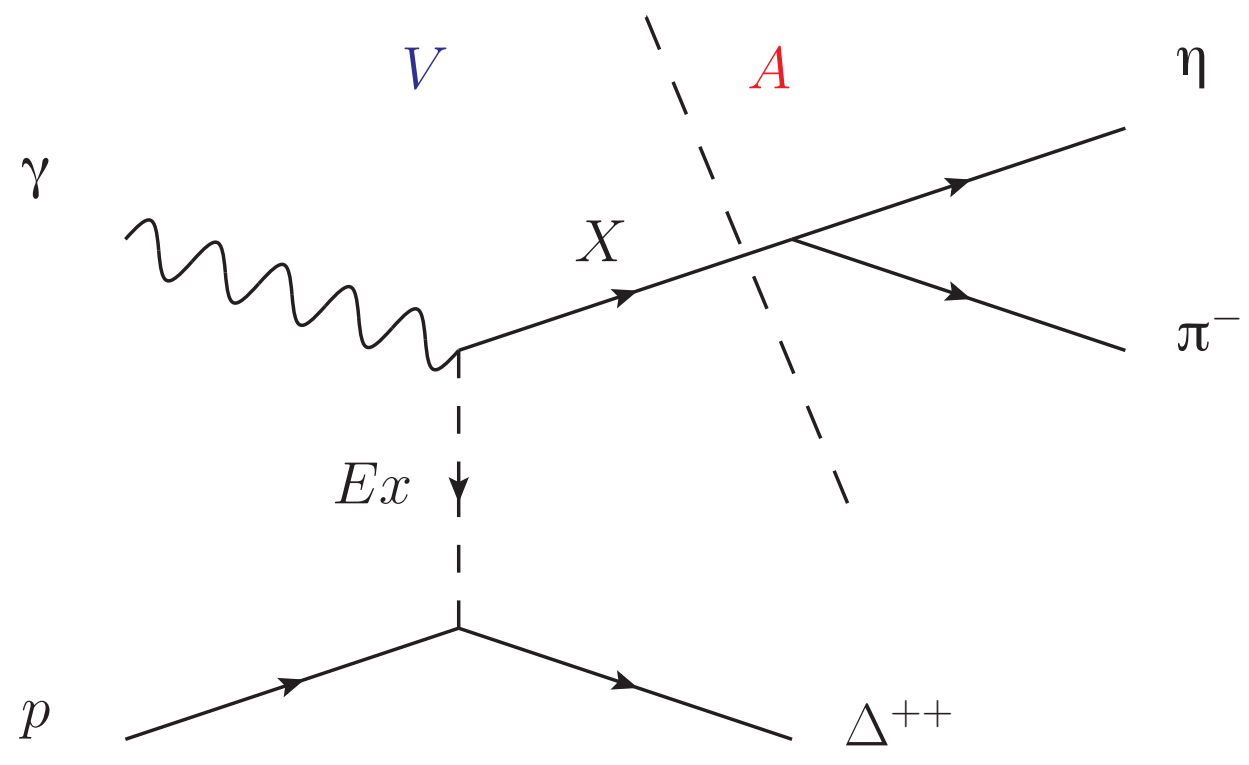

Figure 4.1: Feynman diagram of $\gamma p \rightarrow \Delta^{++} X$ for PWA.

The decay amplitudes, $A$, are calculated from the decay products, $\eta$ and $\pi^{-}$. The production amplitude, $V$, is the fitted parameter and includes contributions from the left side of the dashed line.

- $M$, the projection of $J$ along the beam axis

- $L$, angular momentum of $\eta$ and $\pi^{-}$together

- $I$, isospin of $X$

- $S$, spin of $X$

- $\lambda$, helicity in the rest frame

The total angular momentum, $J$, is the sum of the angular momentum of the $\eta$ and $\pi^{-}$system and the sum of the spins of the $\eta$ and $\pi^{-}$. In this case $\eta$ and $\pi^{-}$are spin-less particles so that

$$
L=L_{\eta}+L_{\pi^{-}}
$$

and

$$
J=L_{\text {total }}+S(=0) .
$$


The parity of the resonance is determined by the intrinsic parity of the $\eta$ and $\pi^{-}$and the relative orbital angular momentum,

$$
P=P_{\eta} P_{\pi^{-}}(-)^{L}
$$

The formalism used in this analysis is based on Chung [29, 30, 31], as used in the E852 [3, 32, 33] and g6c [34] analysis. The resonance is a combination of states and therefore all contributions of waves and interferences of waves are taken into account. The interference is dealt with by the $A$ and $V$ being complex variables.

\subsection{Decay Amplitudes}

To find $A, X$ is described by $J, M, L$, and $S . X$ decays to $\eta$ and $\pi^{-}$where they are described by three vector momenta $\left(p_{\eta}\right.$ and $\left.p_{\pi^{-}}\right)$and spins $\left(S_{\eta}\right.$ and $\left.S_{\pi^{-}}\right)$, as shown in Fig. 4.2 in the lab frame.

In the rest frame of $X, p$ is the momentum of $\eta$ with a direction given by $\theta_{\eta}$ and $\phi_{\eta}$, these are known as the Gottfried-Jackson angles as shown in Fig. 4.3. The decay amplitude is

$$
A\left(\theta_{\eta}, \phi_{\eta}\right)=<\theta_{\eta} \phi_{\eta} \lambda_{\eta} \lambda_{\pi^{-}}\left|\hat{T_{\text {decay }}}\right| J M>
$$

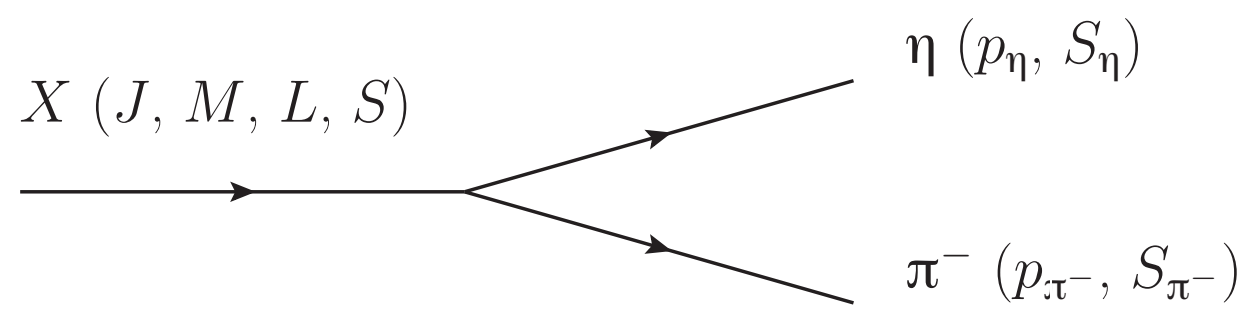

Figure 4.2: Decay of $X$ to $\eta$ and $\pi^{-}$in lab frame. 


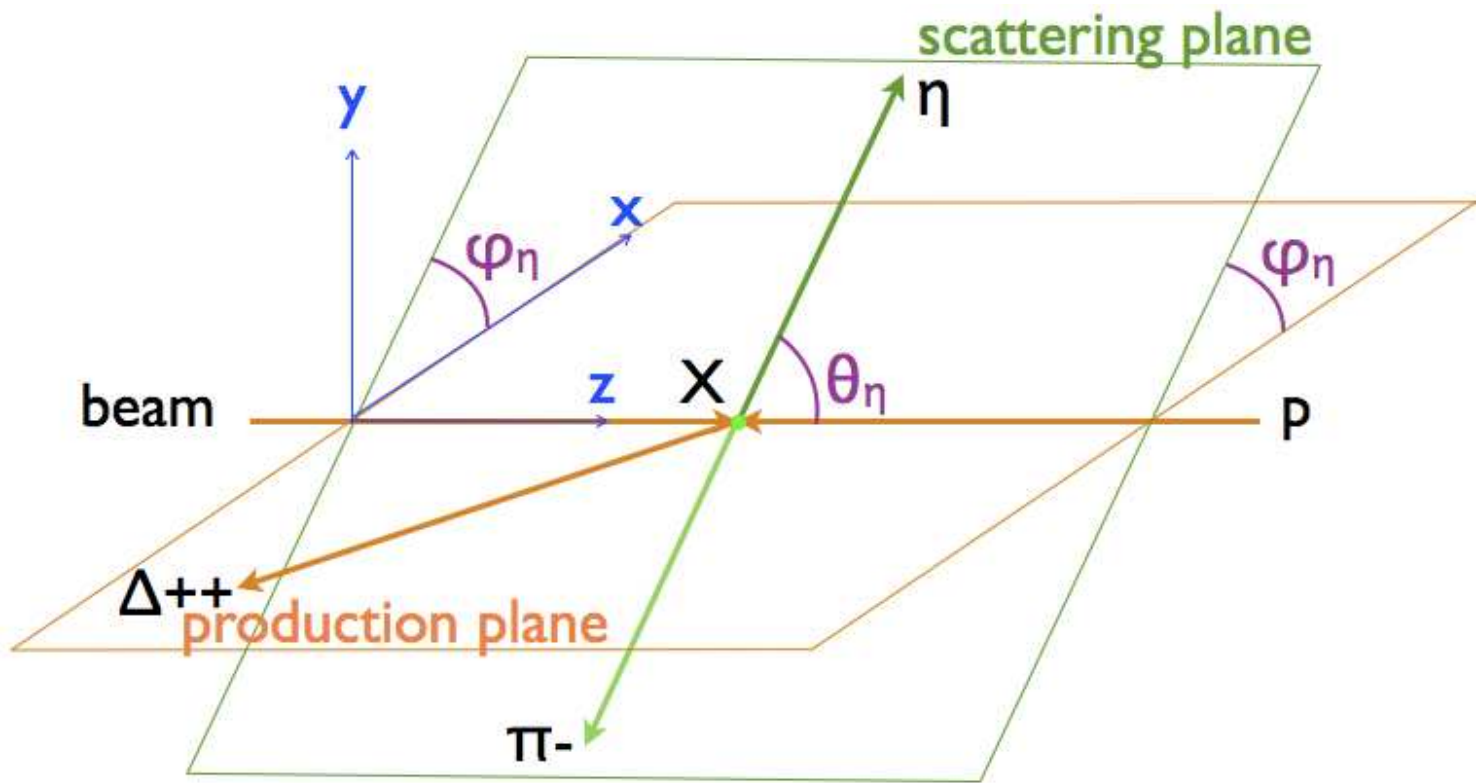

Figure 4.3: Diagram of the Gottfried-Jackson angles.

The production plane is defined by the beam and $\Delta^{++}$vectors in orange. The Gottfried-Jackson angles are defined in the rest frame of the vector meson, $X$, where the $y$-axis is defined as the axis perpendicular to the production plane, and the $z$-axis is defined as the direction of the beam. The $\theta_{\eta}$ of the $\eta$ is the angle between $\hat{z}$ and the momentum vector of the $\eta, \phi_{\eta}$ is the angle between the production plane and the scattering plane of the $\eta$ and $\pi^{-}$. 
where $\hat{T_{\text {decay }}}$ is the transition operator between $\mid \theta_{\eta} \phi_{\eta} \lambda_{\eta} \lambda_{\pi^{-}}>$and $\mid J M>$. Using the relation:

$$
1=\sum_{J, M, L, S}|J M L S><J M L S|
$$

from Ref. [35], the decay amplitude is expanded to

$$
A\left(\theta_{\eta}, \phi_{\eta}\right)=\sum_{J, M, L, S}<\theta_{\eta} \phi_{\eta} \lambda_{\eta} \lambda_{\pi^{-}}|J M L S><J M L S| \hat{T_{\text {decay }}} \mid J M>
$$

The later part, $<J M L S\left|\hat{T_{\text {decay }}}\right| J M>$ is known as the coupling constant, $a_{L S}$, which contains the dynamics of the decay. The factor is absorbed into the production wave that is determined by the fit. $a_{L S}$ is a function of the mass of the resonance and is handled by performing the fits on narrow enough mass bins of the resonance to assume $a_{L S}$ is constant over the width of the bin. The remaining factor,

$$
<\theta_{\eta} \phi_{\eta} \lambda_{\eta} \lambda_{\pi^{-}}\left|J M L S>=<\theta_{\eta} \phi_{\eta} \lambda_{\eta} \lambda_{\pi^{-}}\right| J M \lambda_{\eta} \lambda_{\pi^{-}}><J M \lambda_{\eta} \lambda_{\pi^{-}} \mid J M L S>
$$

is a rotation between the GJ frame $\theta_{\eta}$ and $\phi_{\eta}$ in Fig. 4.4 to $\theta_{\eta}=0$ and $\phi_{\eta}=0$ in Fig. 4.5 followed by a change of basis from $\mid J M \lambda_{\eta} \lambda_{\pi^{-}}>$to $\mid J M L S>$.

The rotation is defined as:

$$
<\theta_{\eta} \phi_{\eta} \lambda_{\eta} \lambda_{\pi^{-}} \mid J M \lambda_{\eta} \lambda_{\pi^{-}}>=\frac{\tilde{J}}{\sqrt{4 \pi}} D_{M \lambda}^{J *}\left(\theta_{\eta}, \phi_{\eta}, 0\right)
$$

where $D_{M \lambda}^{J *}(\alpha, \beta, \gamma)$ is the Wigner $\mathrm{D}$ function [2] and $\tilde{J}=\sqrt{2 J+1}[36]$. The $\gamma$ is an overall phase shift and is taken to be zero in this analysis for simplicity. The change of basis is defined as:

$$
<J M \lambda_{\eta} \lambda_{\pi^{-}} \mid J M L S>=\frac{\tilde{L}}{\tilde{J}}(L 0 S \lambda \mid S \lambda)\left(S_{\eta} \lambda_{\eta} S_{\pi^{-}}-\lambda_{\pi^{-}} \mid S \lambda\right),
$$

where $(L 0 S \lambda \mid S \lambda)$ and $\left(S_{\eta} \lambda_{\eta} S_{\pi^{-}}-\lambda_{\pi^{-}} \mid S \lambda\right)$ are Clebsch-Gordan coefficients from Ref. [2]. The final decay amplitude is

$$
A_{J, M, L, S}\left(\theta_{\eta}, \phi_{\eta}\right)=\sum_{\lambda} \tilde{L} D_{M \lambda}^{J *}\left(\theta_{\eta}, \phi_{\eta}, 0\right)(L 0 S \lambda \mid S \lambda)\left(S_{\eta} \lambda_{\eta} S_{\pi^{-}}-\lambda_{\pi^{-}} \mid S \lambda\right) F_{L}(p) a_{L S}
$$




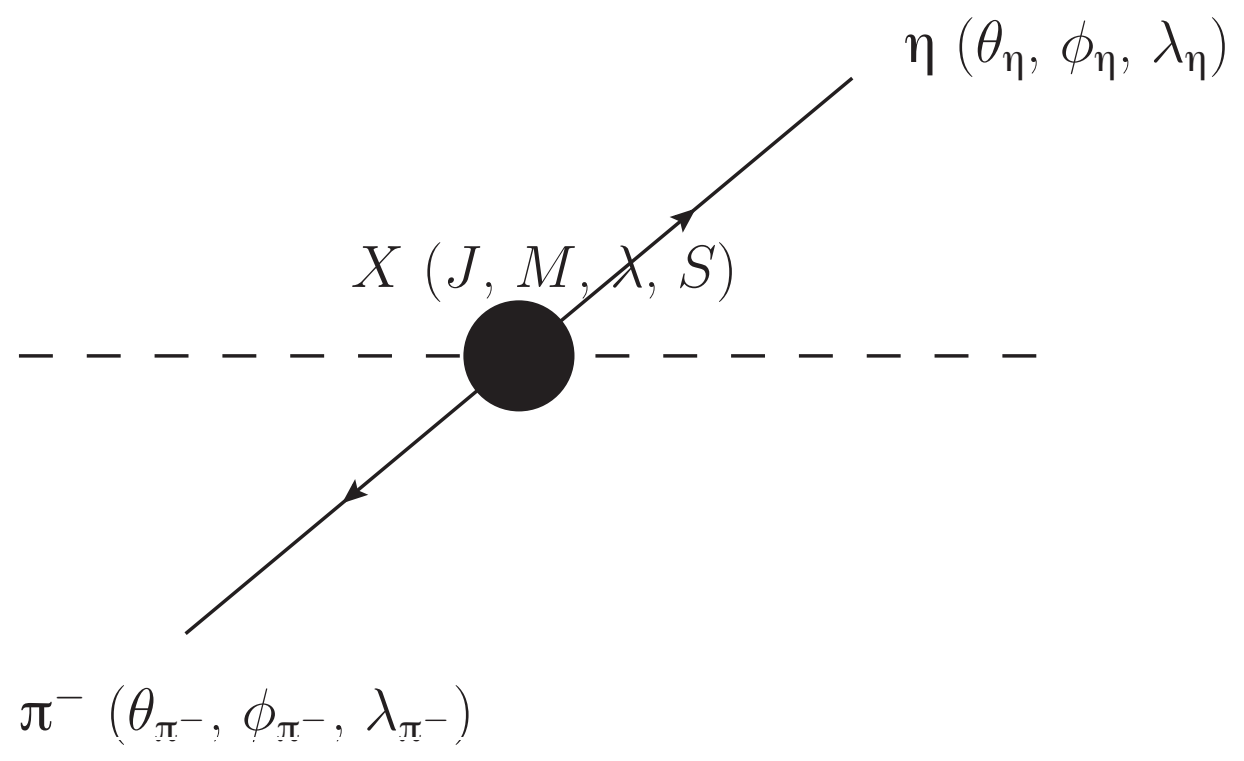

Figure 4.4: Decay of $X$ to $\eta$ and $\pi^{-}$in rest frame of the resonance, with the $\hat{z}$ direction defined as the beam direction.

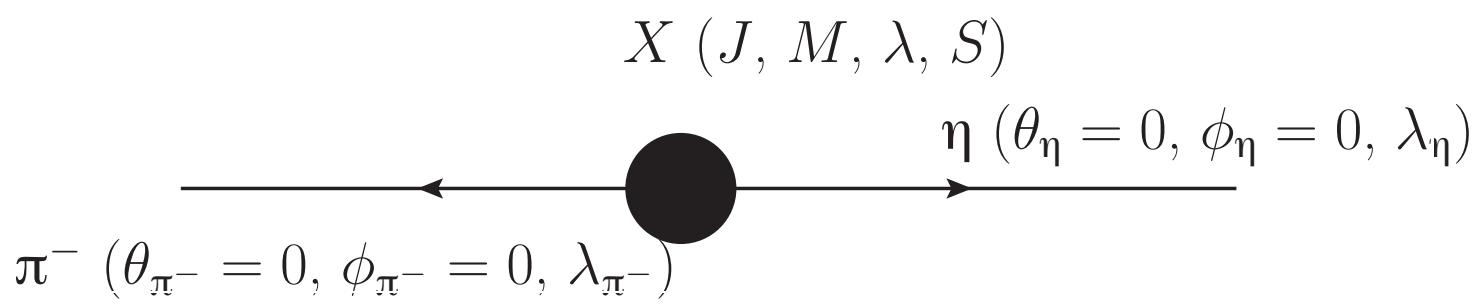

Figure 4.5: Decay of $X$ to $\eta$ and $\pi^{-}$in rest frame of the resonance, with the $\hat{z}$ direction defined as the meson momentum direction. 
where $F_{L}(p)$ is the barrier function, which in this analysis is the Blatt-Weisskopf centrifugal-barrier function for correcting the amplitude near threshold [37]. The barrier function is a function of the breakup momentum and for the first three states of angular momentum $(J)$ are

$$
\begin{gathered}
F_{0}(x)=1, \\
F_{1}(x)=\frac{1}{1+x^{2}},
\end{gathered}
$$

and

$$
F_{2}(x)=\frac{1}{9+3 x^{2}+x^{4}} .
$$

The detailed derivation of the intermediate steps is explained in detailed in Ref. [35] for the decay to two-particle states.

\subsection{Production Amplitude and Intensity}

The production amplitudes are not directly calculated but have to be fit to the data. The $V$ of $X$ is dependent on the quantum states of the beam and the exchange particle (which is dependent on the target and recoil $\Delta^{++}$). The photon contribution of $V$ is expressed using the photon spin density matrix. The photon spin density matrix element $\left(\rho_{\gamma}\right)$ can be taken as an additional product in the intensity distribution,

$$
I\left(\theta_{\eta}, \phi_{\eta}\right)=\sum_{\alpha}\left(V_{\alpha}\left(\theta_{\eta}, \phi_{\eta}\right) A_{\alpha}\left(\theta_{\eta}, \phi_{\eta}\right) \rho_{\gamma} V_{\alpha}^{*}\left(\theta_{\eta}, \phi_{\eta}\right) A_{\alpha}^{*}\left(\theta_{\eta}, \phi_{\eta}\right)\right) .
$$

The amplitudes are constructed as eigenstates of reflectivity to take advantage of the parity conservation in the production process [38]. It reduces the possible number of external spin configurations by a factor of two. Essentially, the sign of the quantum number $M$ becomes the reflectivity, $\epsilon$, and $M$ is now the absolute value of the initial $M$. Breaking the amplitudes into eigenstates of $\epsilon$ results in the intensity distribution 
for a photon beam of:

$$
I\left(\theta_{\eta}, \phi_{\eta}\right)=\sum_{\epsilon} \rho_{\epsilon \epsilon^{\prime}}\left|\sum_{\alpha}{ }^{\epsilon} V_{\alpha}\left(\theta_{\eta}, \phi_{\eta}\right){ }^{\epsilon} A_{\alpha}\left(\theta_{\eta}, \phi_{\eta}\right)\right|^{2},
$$

where ${ }^{\epsilon} V_{\alpha}\left(\theta_{\eta}, \phi_{\eta}\right)$ and ${ }^{\epsilon} A_{\alpha}\left(\theta_{\eta}, \phi_{\eta}\right)$ are the production and decay amplitudes for a given reflectivity, $\epsilon$.

The introduction of reflectivity results in bringing the spin density matrix into a block-diagonal form. The photon spin-density matrix in the reflectivity basis is

$$
\rho_{\epsilon \epsilon^{\prime}}=\left(\begin{array}{cc}
\frac{1}{2}\left(1-P\left[\cos \delta-\sin ^{2} \delta\right]\right) & P \cos \delta \sin \delta \\
P \cos \delta \sin \delta & \frac{1}{2}\left(1-P\left[\cos \delta-\sin ^{2} \delta\right]\right)
\end{array}\right)
$$

where for unpolarized and circularly polarized photon beam, $P=0$ and the $\rho$ matrix is $\frac{1}{2}$ times the identity matrix. The $\epsilon \neq \epsilon^{\prime}$, values of the $\rho_{\epsilon \epsilon^{\prime}}$ matrix are the $\epsilon \epsilon^{\prime}$ interference terms. In the case of a circularly polarized beam, states with different reflectivities cannot interfere with each other. This choice reduces the possible number of external spin configurations by a factor of two and reduces the spin-density matrix to a block-diagonalized form, where there is interference only between amplitudes of the same reflectivity. The decay amplitude rewritten to include reflectivity is defined as

$$
{ }^{\epsilon} A_{\alpha}\left(\theta_{\eta}, \phi_{\eta}\right)=\Theta(M)\left(A_{\alpha}^{M}\left(\theta_{\eta}, \phi_{\eta}\right)-\epsilon P(-1)^{J-M} A_{\alpha}^{-M}\left(\theta_{\eta}, \phi_{\eta}\right)\right)
$$

where $\Theta(M)$ is $\frac{1}{\sqrt{2}}$ or $\frac{1}{2}$ for $M>0$ and $M=0$, respectively [29].

Using the relations explained in Fig. 1.1 and Section 1.4, the production states are in table 4.2. It shows the states as calculated from the production side of the reaction. The resonance is expected to have a total angular momentum of $J=0,1,2$. Table 4.2 shows the mesons related to the states. 


\begin{tabular}{|c|c|c|}
\hline \multicolumn{3}{|c|}{ Initial States } \\
\hline & $I\left(J^{P C}\right)$ & $\left|j_{1}-j_{2}\right| \leq J \leq\left|j_{1}+j_{2}\right|$ \\
\hline$\gamma$ & $0\left(1^{--}\right)$ & \\
\hline $\mathrm{p}$ & $1 / 2\left(1 / 2^{+}\right)$ & $1 / 2 \leq J \leq 3 / 2$ \\
\hline \multicolumn{3}{|c|}{ Final States } \\
\hline$\Delta^{++}$ & $3 / 2\left(3 / 2^{+}\right)$ & \\
\hline$\eta \pi^{-}$resonance & $J=0,1,2$ & \\
\hline \hline
\end{tabular}

\begin{tabular}{|l|l|l|}
\hline wave & $J^{P}(M) \epsilon$ & corresponding particle \\
\hline $\mathrm{S}$ & $0^{+}(0)-$ & $a_{0}(980)$ \\
\hline $\mathrm{P}$ & $1^{-}(0,1) \pm$ & $\begin{array}{l}\boldsymbol{\pi}_{1}(1400) \\
\boldsymbol{\pi}_{1}(1600)\end{array}$ \\
\hline $\mathrm{D}$ & $2^{+}(0,1,2) \pm$ & $a_{2}(1320)$ \\
\hline
\end{tabular}

Table 4.1: Summary of the partial wave states and corresponding meson.

\subsection{Maximum Likelihood Method}

The intensity distribution is a probability distribution over the $\theta$ and $\phi$ of the $\eta$ in the GJ frame. The decay amplitudes are calculated for the wave set but the unknown production amplitudes are varied by the fitter to adjust the predicted intensity distribution to match the observed intensity as a function of $\theta_{\eta}$ and $\phi_{\eta}$. It is done through the extended maximum likelihood fit. During the fitting process the finite acceptance of the detector is taken into account on a wave by wave basis. In other words, each wave contains a decay amplitude with a unique $\theta_{\eta}$ and $\phi_{\eta}$ shape and the acceptance is determined separately for each wave. The formalism has been discussed as a function of $\theta_{\eta}$ and $\phi_{\eta}$. The likelihood fitting is done over a set mass bin of the resonance spectrum shown in the invariant mass of $\eta \pi^{-}$of Fig. 4.6. To maintain large enough statistics per bin, the width of the mass bins to be fit is $50 \mathrm{MeV}$. The mass bins are treated independently of each other as the decay amplitudes are calculated for a wave set over one bin and the production amplitudes are treated unique to that bin. There are no additional corrections to the data beyond what was discussed in Chapter 3 . 


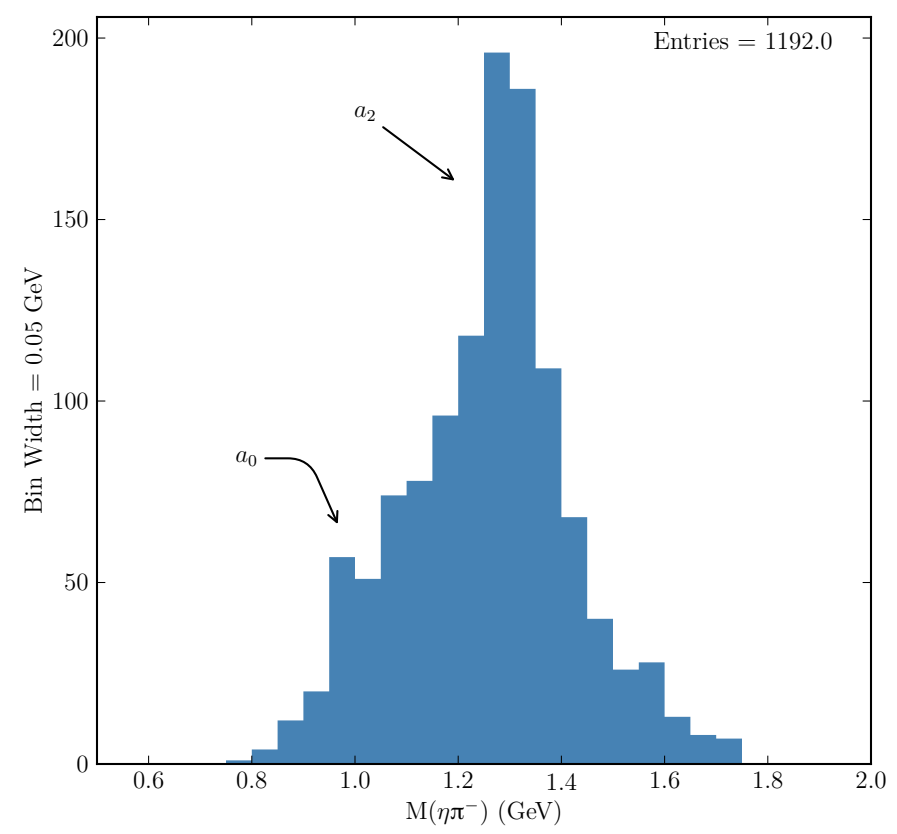

Figure 4.6: Invariant mass of $\eta \pi^{-}$binned for the PWA fit.

The likelihood, $L$, is the product of the probability for each event in the bin. For the partial wave analysis, the probability is the intensity distribution over the variables $\theta_{\eta}$ and $\phi_{\eta}$. The likelihood is defined as:

$$
L=\exp \left(-\bar{N}_{o}\left({ }^{\epsilon} V_{\alpha}\right)\right) \prod_{i}^{N_{o}} I\left(\theta_{\eta i}, \phi_{\eta i}\right),
$$

where $I\left(\theta_{\eta i}, \phi_{\eta i}\right)$ is the intensity distribution for an individual bin in the angles $\phi_{\eta}$ and $\theta_{\eta}$ from equation 4.24 [15]. $\quad \bar{N}_{o}\left({ }^{\epsilon} V_{\alpha}\right)$ is the average number of events observed if the exact experiment was repeated several times for a given ${ }^{\epsilon} V_{\alpha} . \bar{N}_{o}\left({ }^{\epsilon} V_{\alpha}\right)$ takes into account the acceptance of the experiment and is evaluated using Monte Carlo simulations to model the reaction in the detector configuration.

Since the likelihood function is a product of probabilities over the number of events in a given $\eta \pi^{-}$mass bin, it becomes large very quickly. For practical reasons it is the $-\ln (L)$ that is minimized during the fitting procedure with the production amplitudes varied to find the minimum $-\ln (L)$. Expanding the likelihood function 
yields:

$$
\begin{aligned}
\ln L= & \sum_{i}^{N_{o}} \ln \sum_{\epsilon, \epsilon^{\prime}, \alpha, \alpha^{\prime}} \rho_{\epsilon \epsilon^{\prime}} \underbrace{{ }^{\epsilon} V_{\alpha} \epsilon^{\prime} V_{\alpha^{\prime}}^{*}}_{\text {fit parameters }}{ }^{\epsilon} A_{\alpha}\left(\theta_{\eta i}, \phi_{\eta i}\right){ }^{\epsilon^{\prime}} A_{\alpha^{\prime}}^{*}\left(\theta_{\eta i}, \phi_{\eta i}\right) \\
& -N_{o} \frac{N_{r}}{N_{\eta}} \sum_{\epsilon, \epsilon^{\prime}, \alpha, \alpha^{\prime}} \underbrace{{ }^{\epsilon} V_{\alpha} \epsilon^{\prime} V_{\alpha^{\prime}}^{*}}_{\text {fit parameters }}{ }^{\epsilon \epsilon^{\prime}} \Phi_{\alpha \alpha^{\prime}}^{\eta},
\end{aligned}
$$

where

$$
{ }^{\epsilon \epsilon^{\prime}} \Phi_{\alpha \alpha^{\prime}}^{\eta}=\frac{1}{N_{\eta}} \sum_{i}^{N_{\eta}} \rho_{\epsilon \epsilon^{\prime}}{ }^{\epsilon} A_{\alpha}\left(\theta_{\eta i}, \phi_{\eta i}\right){ }^{\epsilon} A_{\alpha^{\prime}}^{*}\left(\theta_{\eta i}, \phi_{\eta i}\right),
$$

[15] and ${ }^{\epsilon \epsilon^{\prime}} \Phi_{\alpha \alpha^{\prime}}^{\eta}$ is the normalized acceptance integral calculated from the amplitudes of the accepted simulated data. From the simulation, $N_{r}$ is the number of raw events and $N_{\eta}$ is the number of events accepted. $N_{o}$ is the number of observed events in the data. The fitting algorithm is broken down into a series of sums,

$$
\begin{gathered}
-\ln L=-\sum_{i}^{N_{o}}\left[W_{i}\right]+n[N \text { orm }] \\
W=\ln \sum_{\epsilon, \epsilon^{\prime}, \alpha, \alpha^{\prime}} \underbrace{{ }^{\epsilon} V_{\alpha} \epsilon^{\prime} V_{\alpha^{\prime}}^{*}}_{\text {fit parameters }} \underbrace{\epsilon^{\epsilon} A_{\alpha}\left(\theta_{\eta i}, \phi_{\eta i}\right){ }^{\epsilon^{\prime}} A_{\alpha^{\prime}}^{*}\left(\theta_{\eta i}, \phi_{\eta i}\right)}_{\text {calc. amp. }} \\
N o r m=\frac{N_{r}}{N_{\eta}} \sum_{\epsilon, \epsilon^{\prime}, \alpha, \alpha^{\prime}}{ }^{\epsilon} V_{\alpha}{ }^{\epsilon^{\prime}} V_{\alpha^{\prime}} \underbrace{\epsilon \epsilon^{\prime} \Phi_{\alpha \alpha^{\prime}}^{\eta}}_{\text {acc. integral }}
\end{gathered}
$$

where ${ }^{\epsilon \epsilon^{\prime}} \Phi_{\alpha \alpha^{\prime}}^{\eta}$ is the normalization integral calculated from the amplitudes of the accepted simulated events and is used as an acceptance correction value. The ${ }^{\epsilon} A_{\alpha^{\prime}}^{*}\left(\theta_{\eta i}, \phi_{\eta i}\right)$ are the amplitudes calculated for all data events.

\subsection{Partial Wave Fitting Procedure}

The PWA formalism was coded in to a Java program by the author, with the assistance of Dennis Weygand. The Java package is setup with the same structure as the $\mathrm{C}++$ version documented in [37]. There are three main components of the PWA package: partial wave decay amplitude calculation $\left({ }^{\epsilon} A_{\alpha^{\prime}}^{*}\left(\tau_{i}\right)\right)$, accepted and 
raw normalization integrals ( ${ }^{\epsilon} \Phi_{\alpha \alpha^{\prime}}^{\eta}$ ), and fitting. The program is designed to call the MINUIT minimization package in the CERNLIB library and fits equation 4.21 to determine the real and imaginary parts of the production amplitude, $\operatorname{Re}\left({ }^{\epsilon} V_{\alpha}\right)$ and $\operatorname{Im}\left({ }^{\epsilon} V_{\alpha}\right)$, respectively, for each of the partial wave states. There are twice the number of fit parameters as waves due to the minimizer being used for the fit accepting real parameters only. The acceptance corrected yield for an individual mass bin is defined by

$$
N=N_{0} \frac{N_{r}}{N_{\eta}} \sum_{\epsilon, \alpha, \alpha^{\prime}}{ }^{\epsilon} V_{\alpha}{ }^{\epsilon} V_{\alpha^{\prime}}^{*}{ }^{\epsilon} \Phi_{\alpha \alpha^{\prime}}^{\eta}
$$

where ${ }^{\epsilon} \Phi_{\alpha \alpha^{\prime}}^{\eta}$ is raw normalization integral calculated from the amplitudes of the raw generated events. The acceptance corrected yield of a single state is defined by

$$
N=N_{0} \frac{N_{r}}{N_{\eta}}\left|{ }^{\epsilon} V_{\alpha}\right|^{2}{ }^{\epsilon} \Phi_{\alpha \alpha}^{\eta} .
$$

The acceptance corrected yields plotted as a function of resonance mass are used to extract a mass and width of the peak in a partial wave state. This process is referred to as a mass-dependent fit as later discussed in section 4.6.3. The phase difference between two wave states is

$$
\Delta \Phi=\arctan \left(\frac{\operatorname{Im}\left({ }^{\epsilon} V_{\alpha}{ }^{\epsilon} V_{\alpha^{\prime}}^{*}\right)}{\operatorname{Re}\left({ }^{\epsilon} V_{\alpha}{ }^{\epsilon} V_{\alpha^{\prime}}^{*}\right)}\right) .
$$

The phase difference between the complex variables, ${ }^{\epsilon} V_{k \alpha}$ and ${ }^{\epsilon} V_{k \alpha^{\prime}}^{*}$, can show interference between wave states and can be fitted for the masses and widths of these states. The systematic errors for the acceptance corrected yield and the phase difference is explained in detail in Appendix A.

\subsection{Experimental Acceptance}

The experimental acceptance is determined by generating $\Delta^{++} X$ events with $\Delta^{++} \rightarrow$ $p \pi^{+}, X \rightarrow \eta \pi^{+}$, and $\eta \rightarrow \gamma \gamma$. The $\Delta^{++}$is generated with a mass and width of 
1.233 and $0.1 \mathrm{GeV}$, respectively, to match the $p \pi^{+}$mass distribution of the data. The decay of $X$ to $\eta \pi^{-}$is such that the $\theta$ and $\phi$ of the $\eta$ and $\pi^{-}$in the GJ frame is isotropic. The mass of $X$ was generated over the $\eta \pi^{-}$invariant mass range from 650 to $1700 \mathrm{MeV}$ with $10-\mathrm{MeV}$-wide bins containing 500k events per bin, to be merged to the desired bin width. Generating events in mass bins narrower than the size used in the fit enables the ability to change bin widths without the need to rerun the simulation and to minimize the effect of bin migration. The effect is the result of the finite mass resolution of the experiment resulting in events generated in one bin occupying neighboring bins. Merging $10-\mathrm{MeV}$-wide bins to the desired width will remove most, but not all, of this effect. To match the distributions of the simulation to the experimental data, the events were simulated with a $t$ slope of 3 but reconstructed to be 2.7 after passing the detector simulation as shown in Fig. 4.7, very close to the value as calculated from the $t$ slope of the $a_{2}$ mass range with sideband subtraction shown in Fig. 4.8. The change in the slope is because of limits in the detector acceptance of forward going tracks.

The generated events are fed into GSIM where the particles are swum through the detector packages and detector responses are simulated. The process is the standard CLAS simulation procedure. To realistically model the response of the detectors, the GPP program is used to smear the detector signals from the output of GSIM to match the experimental resolution of the detectors used to take the data. GSIM and GPP output simulated detector responses and the a1c package is used to reconstruct the simulated tracks. It is the same program used to reconstruct the data and uses the same experiment-specific settings. The final accepted events include the analysis cuts discussed in the previous chapter. The GSIM, GPP, and a1c program options used are the standard set, agreed upon for the g12 run period based on the experimental configurations of the setup and parameters used in the processing of the data [39]. 


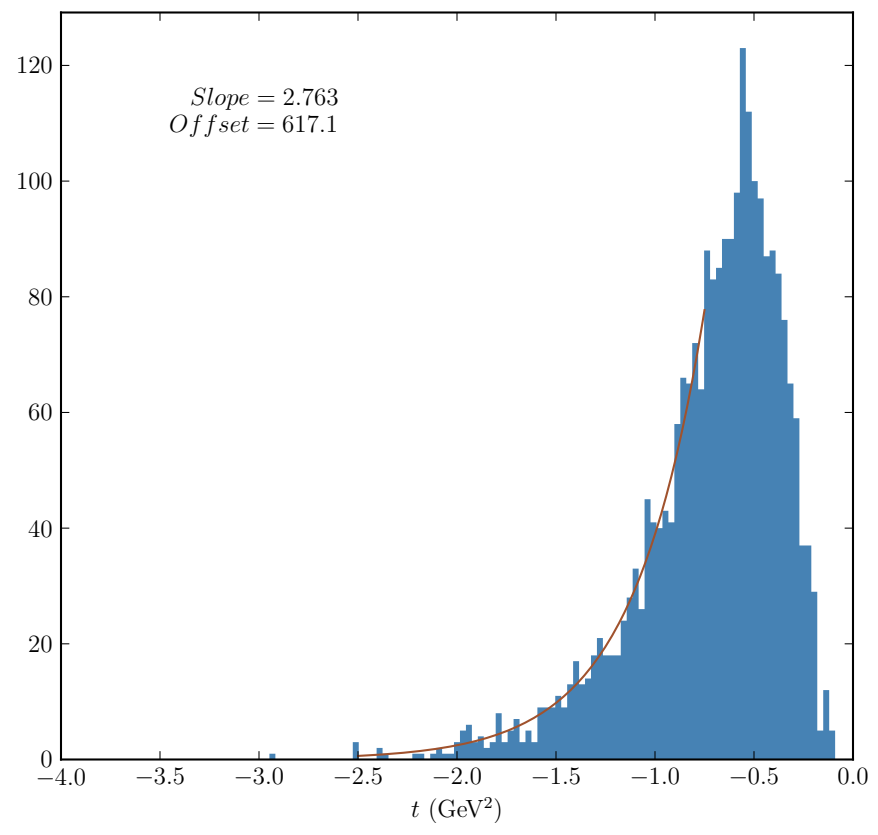

Figure 4.7: $t$ distribution of the simulation.

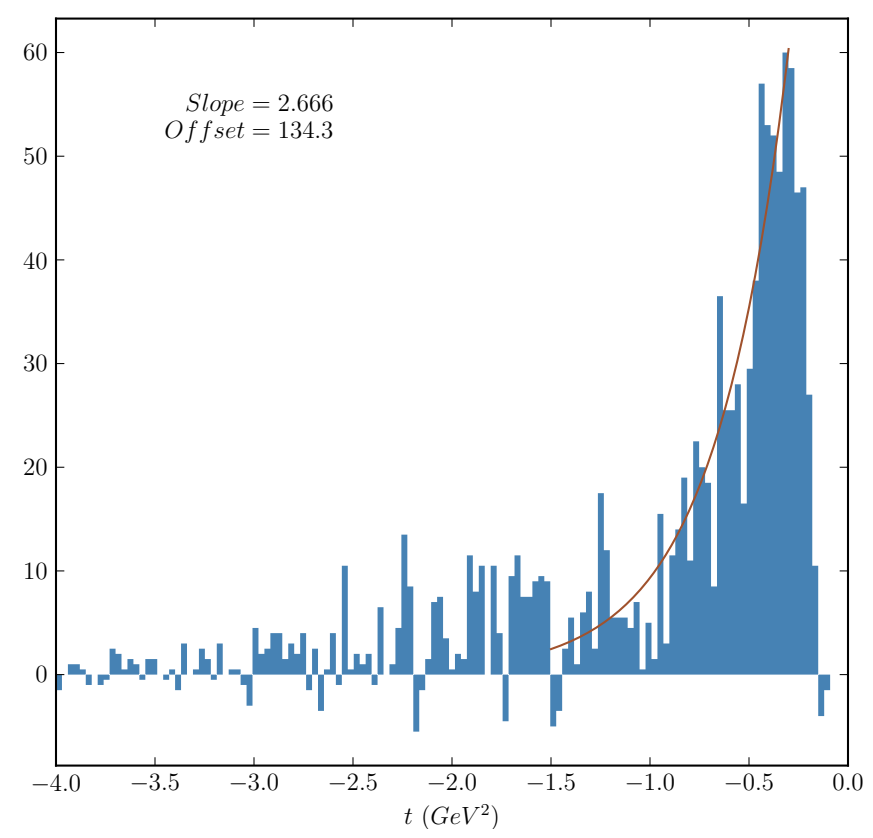

Figure 4.8: $t$ distribution of the data after sideband subtraction. 


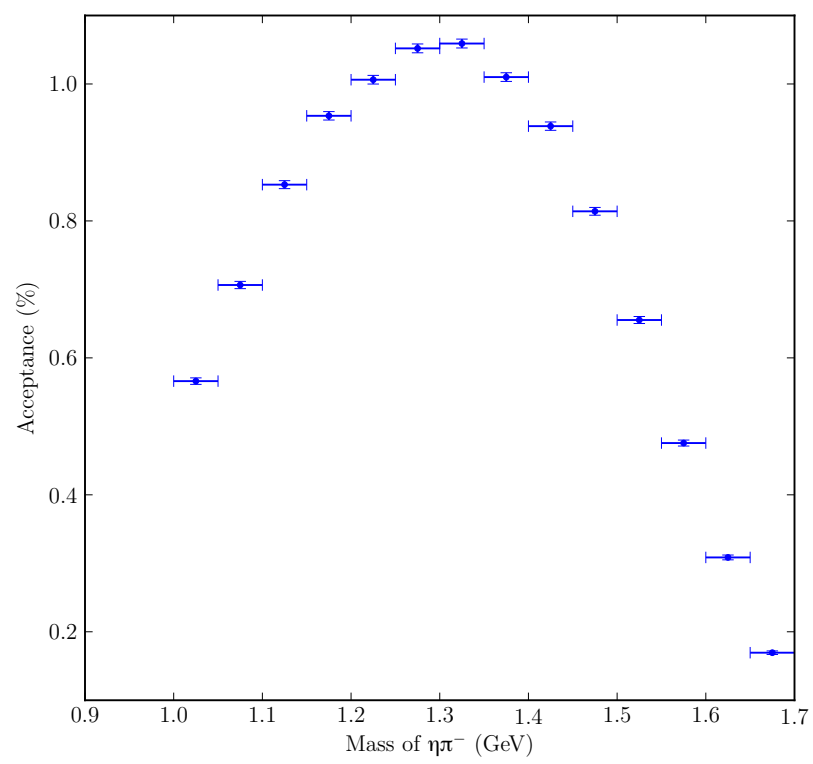

Figure 4.9: Acceptance of $p \pi^{+} \pi^{-} \gamma \gamma$ over the invariant mass of $\eta \pi^{-}$.

Additionally, the beam energy spectrum illustrated in Fig. 3.2 was used to generate the shape of the simulated beam energy spectrum to account for the cross section as a function of beam energy and make the mass-dependent fits a function of the resonance parameters only. The overall acceptance from 1.0 to $1.7 \mathrm{GeV}$ of the $\eta \pi^{-}$ invariant mass is $0.7 \%$, with a maximum acceptance between 1.2 to $1.4 \mathrm{GeV}$ of $1.03 \%$ (shown in Fig. 4.9).

\subsection{Results}

The final data set includes 1197 events and the fit was applied to $50-\mathrm{MeV}$-wide $\eta \pi^{-}$ invariant mass bins, with the largest mass bin containing 195 events between 1.25 to $1.3 \mathrm{GeV}$ (Fig. 3.22). The fits are calculated in two stages. The first is the PWA and includes no assumptions of masses or widths of states. The PWA is executed on individual mass bins of the resonance, $\eta \pi^{-}$, and assumes the amplitudes are constant over the beam-energy range and momentum-transfer range. 


\subsubsection{Wave Set}

In theory, an infinite number of partial waves could be included in the fit and it is the fitter that finds the proportion of the individual partial waves. With limited statistics, only a small selection of waves can be included in the fit at one time. For the meson mass range of 1.0 to $1.6 \mathrm{GeV}$, the higher order states (in this case, any state with $J \geq 3$ ) are not expected so the included states are $J<3$. For $\pi$ exchange, the expected partial wave set is $P_{1}$ and $D_{1}$, with positive and negative reflectivity. The set is the base set and was included in all the fit combinations. The $S_{0}$ is included to absorb the background that is not described by the other partial waves and to absorb any contributions from the tail of the $a_{0}(980) \rightarrow \eta \pi^{-}$that may have survived the $t$ cut. The $S$ wave has an isotropic intensity where the $P$ and $D$ waves are not. The wave state and corresponding particles are shown in Table 4.2. The invariant mass of the $\eta \pi^{+}$shows a peak at the mass of the $a_{0}(980)$ but the acceptance is also peaked in the same region as shown in Fig. 3.23. The background cuts limited the $\eta \pi^{+}$background as discussed in section 3.7 .4 but there will still be some leakage that is expected to be absorbed into the $S_{0}$ wave.

With the circularly polarized photon beam, the positive and negative reflectivity states are expected to have equal contributions. From the Wigner D functions of the decay amplitude, the $\phi_{\eta}$ dependence is dictated by the reflectivity of the states. The sum of two Wigner D function with the same $J^{P C} M$ but opposite $\epsilon$ cancels out the $\phi_{\eta}$ dependence resulting in a flat distribution of intensity over $\phi_{\eta}$, this is seen in the data as later shown in the comparison of the data to prediction for the $\phi_{\eta}$ in section 4.6.2. Initially fits were produced for independent reflectivities. The resulting yields of positive and negative reflectivities were within the error bars of each other but the fits were less stable and the fitter started producing errors in the covariance 


\begin{tabular}{|l|l|l|}
\hline wave & $J^{P} M \epsilon$ & corresponding particle \\
\hline $\mathrm{S}$ & $0^{+} 0^{-}$ & $a_{0}(980)$ \\
\hline $\mathrm{P}$ & $1^{-} 1\left(^{ \pm}\right)$ & $\pi_{1}(1400)$ \\
& & $\pi_{1}(1600)$ \\
\hline $\mathrm{D}$ & $2^{+} 1\left(^{ \pm}\right)$ & $a_{2}(1320)$ \\
\hline
\end{tabular}

Table 4.2: Summary of the partial wave states used in the final fit.

matrix calculations as the number of waves increased. As a result of the fitter errors, reflectivities were tied together.

\subsubsection{PWA: Mass-Independent Fit}

The mass-independent fits have shown a large contribution from the $J^{P}|m|=2^{+} 1$ state, with defined peaks around the mass of the $a_{2}(1320)$ as expected from the quark model. The contributions from other states are not as well defined or structured and are discussed further in section 4.6.3. The mass-independent fit was followed by the mass-dependent fit of the wave intensities to extract the masses and widths of any possible states. The result is the $a_{2}(1320)$ was reconstructed with a width ranging from 0.128 to $0.174 \mathrm{GeV}$ and a mass of about $1.32 \pm 0.04 \mathrm{GeV}$. The details of the results are discussed in the following section.

The PWA was preformed on a bin-by-bin basis of the $\eta \pi^{-}$invariant mass with the fit parameters of each bin independent of the previous bin. For a given set of waves in each fit, ten fits were performed using random initial parameters and the final solution was chosen to be the result with the highest maximum likelihood value. Reiterating the fit avoids selecting a fit that could have found a local maxima, missing the best solution. The fits including waves $J^{P}|m|=1^{-} 0$ and $2^{+} 0$ resulted in less than $5 \%$ of the events going to each of these waves, $P_{0}$ and $D_{0}$, and showed no effect on the expected waves so the waves were left out of the final mass-independent fit. 
Appendix A includes the results of the additional PWA fits including $J^{P}|m|=1^{-} 0$ and $2^{+} 0$.

In all fits the $J^{P}|m|=2^{+} 1$ is the dominant wave and shows a clear peak between 1.3 and $1.35 \mathrm{GeV}$ as shown in Figs. 4.10(a), A.1(b), and A.3(b). The final fit, shown in Figs. 4.10, 4.11 and 4.12, includes the expected waves and $S$-wave background, $2^{+} 1,1^{-} 1$, and $0^{+} 0^{-}$. No structure is seen in the $1^{-} 1$ spectrum in Fig. $4.10(\mathrm{~b})$. Any possible resonance structure is obscured by the large error bars in the $1^{-} 1$ spectrum indicating no statistically significant structure. The phase difference of $1^{-} 1$ and the $2^{+} 1$ shows a decrease expected from the $2^{+} 1$ but is followed by an increase that could be a sign of $1^{-1}$ interference as shown in Fig. 4.12(a). There is a broad peak in the $0^{+} 0^{-}$wave in Fig. 4.11(a). This peak is possibly due to a leak from $2^{+} 1$ or the tail of an $a_{0}(980)$ from $\gamma p \rightarrow \Delta / N^{0} a_{0}$. The phase difference between the $0^{+} 0$ and the $2^{+} 1$ waves shown in Fig. 4.12(b) is $\pm \pi$, occurring consistently in every fit. The phase can be rotated by a multiple of $2 \pi$ and still be the same solution.

\section{Fit Quality}

Although there are multiple solutions to any PWA, not all of the solutions are the ideal fit to the data. To check the quality of the PWA fit, the solution is used to weight generated events, taking wave angle distributions and detector acceptance into account. In equation 4.24 the $V$ and $A$ are known and this equation is used to calculate the weight for each event, $w_{i}$. Taking the dividend of the the weight and the maximum weight, $w_{\max }$, gives the probability of the event. If

$$
r>\frac{w_{i}}{w_{\max }}
$$

is satisfied, the event is kept, where the $r$ is random number between 0 and 1 . The acceptance was taken into account through the use of the experimental acceptance 


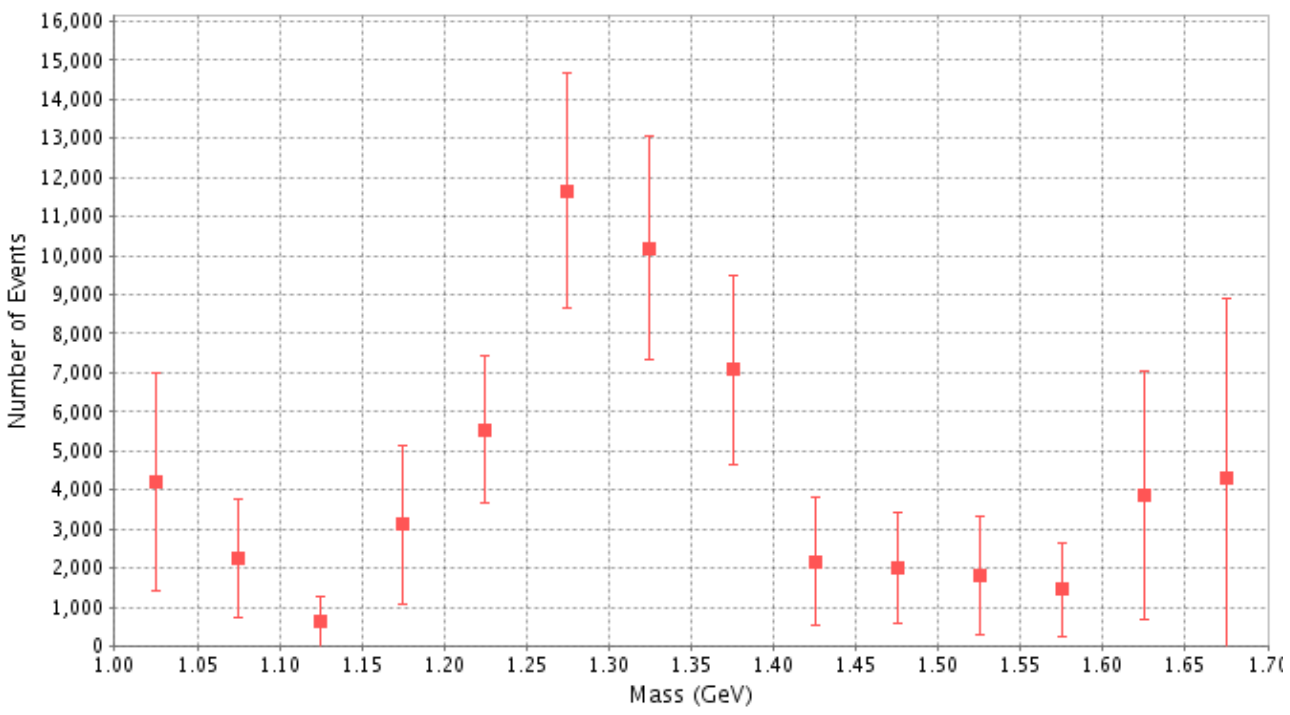

(a) Intensity of $2^{+} 1$ ( $D$-wave)

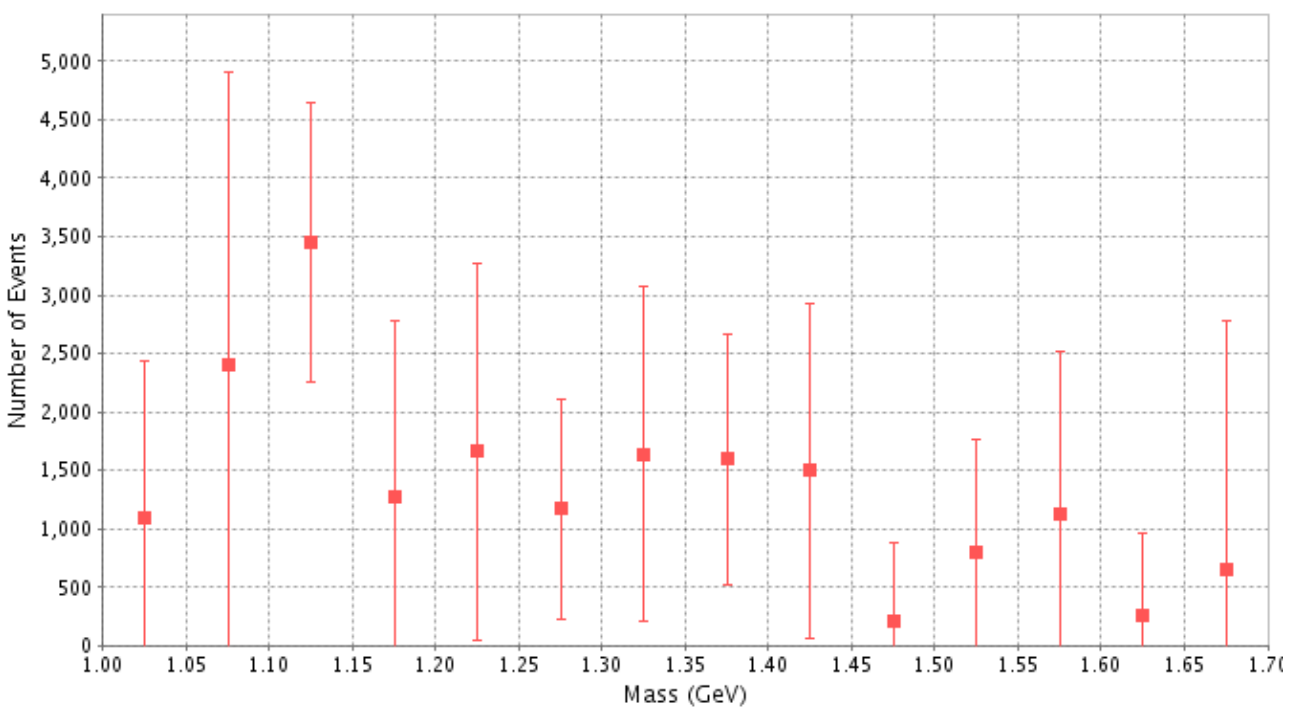

(b) Intensity of $1^{-1}$ ( $P$-wave)

Figure 4.10: Intensity distributions of final wave set. Using a fit with the partial waves $2^{+} 1,1^{-} 1$, and $0^{+} 0^{-}$. 


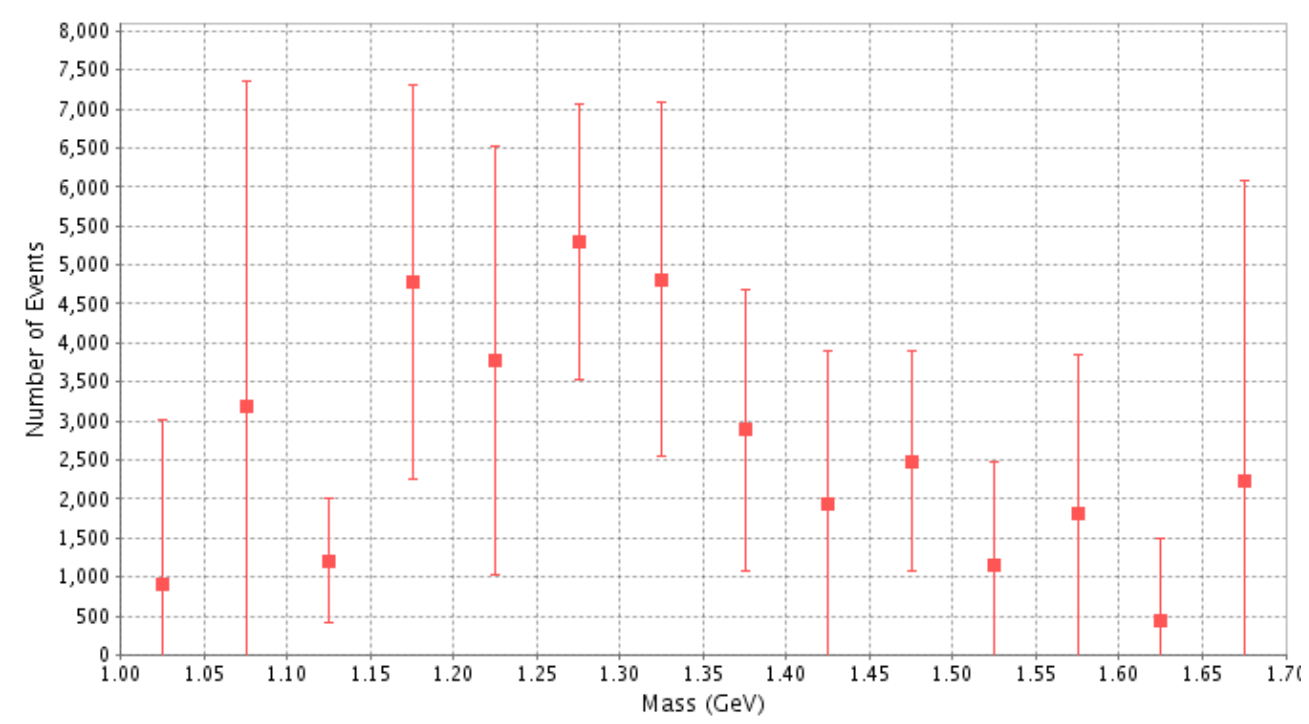

(a) Intensity of $0^{+} 0^{-}(S$-wave $)$

Figure 4.11: Intensity distributions of final wave set. Using a fit with the partial waves $2^{+} 1,1^{-} 1$, and $0^{+} 0^{-}$.

procedure in section 4.5. By comparing the weighted and real data distributions, the quality of the fit is assessed.

In Fig. 4.13 the GJ angles are compared between the data and the prediction from the PWA solution. The $\phi_{\eta}$ of the data and the weighted events are both isotropic as expected from circularly polarized photons. For the $\cos \theta_{\eta}$ of the data, the lack of events in the region of $\cos \theta_{\eta}<0$ is because of the acceptance of CLAS. The $\chi^{2} / d o f$ between the data and the prediction is 0.0133 . The invariant mass distributions of the data and prediction are shown in Figs. 4.14 and 4.15. The conclusion of the mass-independent fit is the PWA solution describes the data very well for the GJ angles, but just as importantly, it also describes the invariant masses that are not a part of the fit. In the generated events the invariant mass distributions of the $p \pi^{+}$ and $\eta \pi^{-}$were used to generate the data set. Figs. 4.14 and 4.15 show the invariant mass spectra not specified by the event generation. From Figs. 4.14 and 4.15, the $\chi^{2} / d o f$ for the $p \pi^{+} \eta$ is $0.3411, p \eta$ is $0.3287, p \pi^{+} \pi^{-}$is 0.5233 , and $\pi^{+} \eta$ is 0.2395 . 


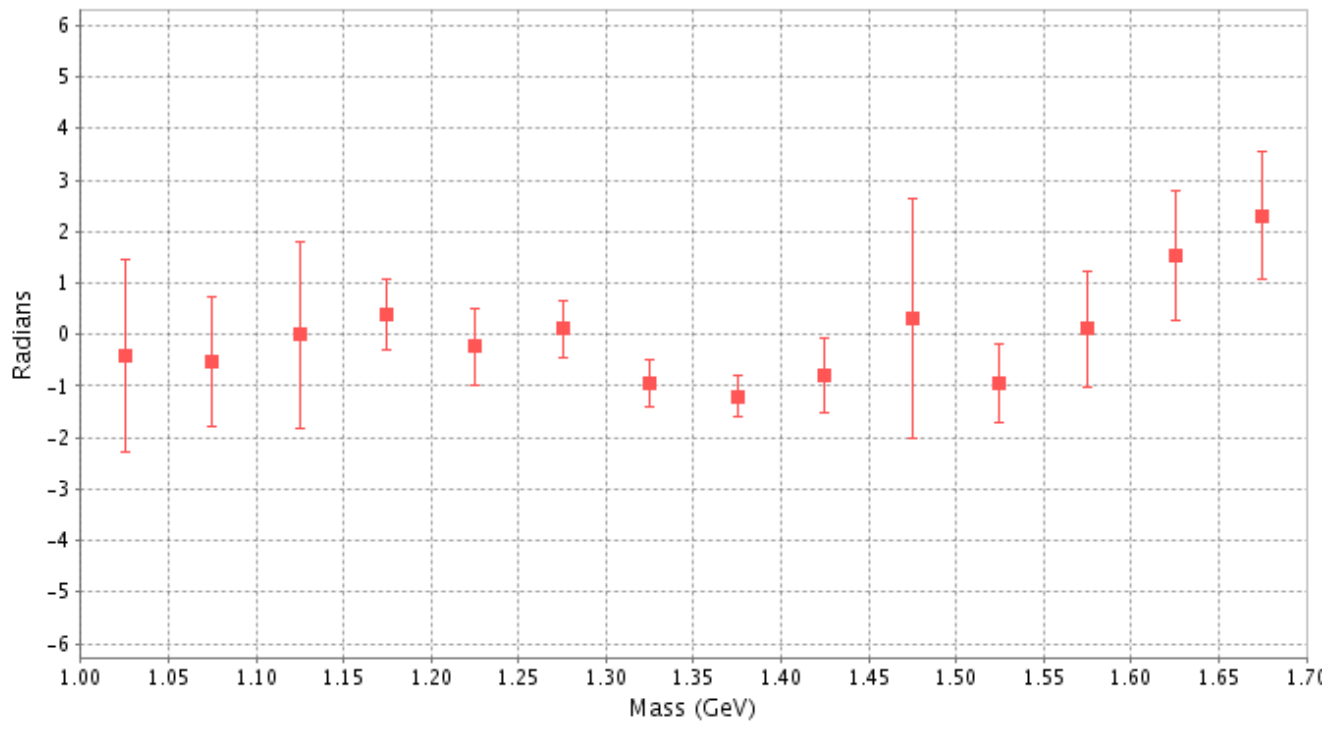

(a) Phase Difference of $1^{-} 1$ and $2^{+} 1$

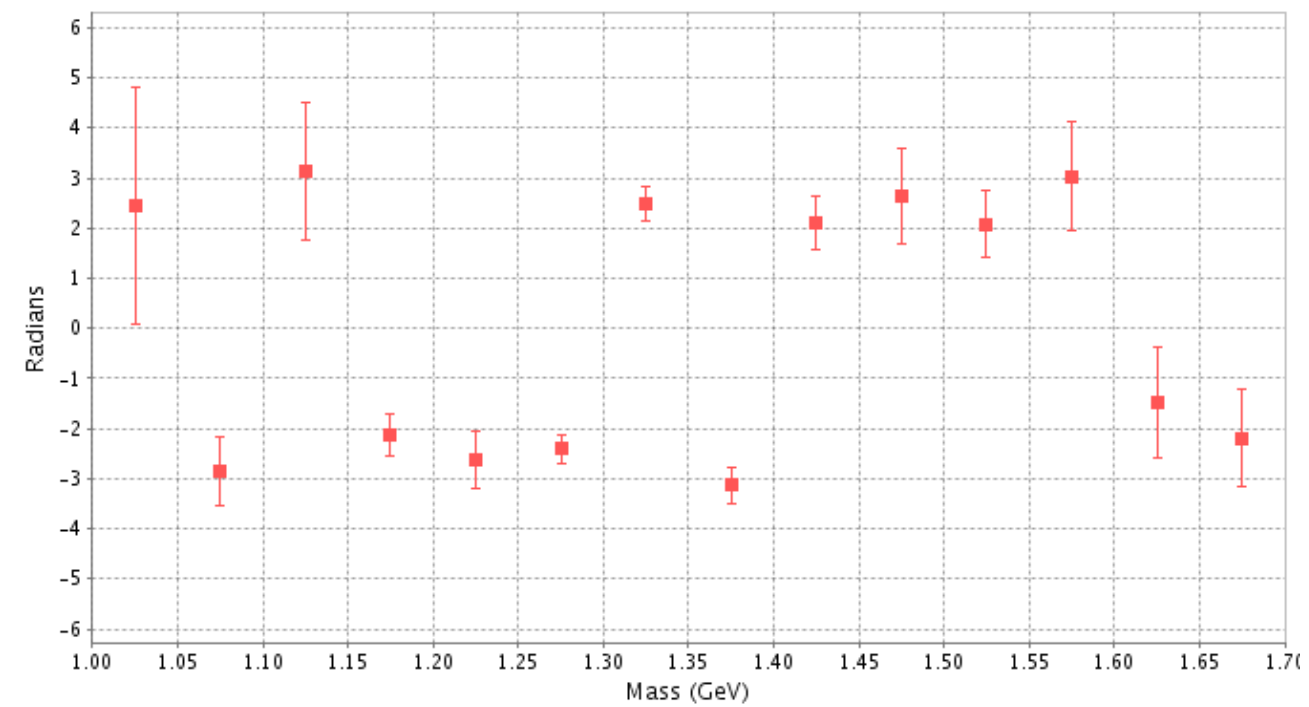

(b) Phase Difference of $0^{+} 0$ and $2^{+} 1$

Figure 4.12: Phase differences of final wave set. Using a fit with the partial waves $2^{+} 1,1^{-} 1$, and $0^{+} 0^{-}$. 


\subsubsection{Mass-Dependent Fit}

To understand the nature of the $P$ and $D$ waves observed in this experiment, a massdependent fit is applied to the results of the PWA intensity and phase difference distributions. This fit was carried out over the $\eta \pi^{-}$mass range of 1.0 to $1.7 \mathrm{GeV}$. The ground state wave in this analysis corresponds to the $a_{2}$, with a mass and width of 1.32 and $0.107 \mathrm{GeV}[2]$, respectively. The bin width is $50 \mathrm{MeV}$ resulting in the width of the $a_{2}$ to be two bins wide, as seen in Fig. 4.6. The result is the fitted width of the $a_{2}$ is expected to be wider than the PDG value. The $P$ and $D$ wave decay amplitudes are treated as resonant and use the relativistic Breit-Wigner forms for the amplitudes. The relativistic Breit-Wigner amplitude for a resonance of mass $m_{0}$ and width of $\Gamma_{0}$ over the mass spectrum $m$ is

$$
a_{J}^{B W}=\frac{-i m_{0} \Gamma\left(m_{0}\right)}{\left(m^{2}-m_{0}^{2}\right)+i m_{0} \Gamma(m)}
$$

[2], where the mass-dependent width, $\Gamma(m)$, is defined as

$$
\Gamma_{J}(x)=\Gamma_{0} \frac{m_{0}}{x}\left(\frac{k(x)}{k\left(m_{0}\right)}\right)^{2 J+1} \frac{F_{J}(R k(x))}{F_{J}\left(R k\left(m_{0}\right)\right)}
$$

[40], where $R$ is the interaction radius of $5 \mathrm{GeV}^{-1} . F_{J}(x)$ is the Blatt-Weisskopf angular momentum barrier function,

$$
\begin{gathered}
F_{0}(x)=1 \\
F_{1}(x)=\frac{1}{1+x^{2}} \\
F_{2}(x)=\frac{1}{9+3 x^{2}+x^{4}},
\end{gathered}
$$

and $k$ is the break-up momentum in the resonance rest frame of a given mass,

$$
k(x)=\frac{x}{2}\left(1-\frac{\left(m_{a}+m_{b}\right)^{2}}{x^{2}}\right)^{1 / 2}\left(1-\frac{\left(m_{a}-m_{b}\right)^{2}}{x^{2}}\right)^{1 / 2}
$$


[40]. The break-up momentum is the momentum for a particle of mass to decay to particles of masses $m_{a}$ and $m_{b}$ in the rest frame. The intensity distribution for a single wave is

$$
N=\left|a_{J}\right|^{2}
$$

where

$$
a_{J}=C_{r e} a_{J}^{B W}\left(m_{0}, \Gamma_{0}, m\right)
$$

and the fit parameters are a normalization constant, $C_{r e}$, mass of the resonance, $m_{0}$, and width of the resonance, $\Gamma_{0}$. The mass-dependent phase shift between two partial waves is

$$
\Delta \phi=\arctan \frac{\operatorname{Im}\left(a_{J} a_{J^{\prime}}^{*}\right)}{\operatorname{Re}\left(a_{J} a_{J^{\prime}}^{*}\right)}
$$

where the fitted phase function is

$$
\Delta \Phi=\delta+\Delta \phi
$$

with $\delta$ as a fitted constant, used as mass-independent phase shift.

The mass-dependent fits were broken down into four types. Type 1 is a fit of the intensity of the $2^{+} 1$ for the $a_{2}$, illustrated in Fig. 4.16 with a final mass and width of $1.32 \pm 0.01$ and $0.154 \pm 0.011 \mathrm{GeV}$, respectively. The fit was used as the initial mass and width for the rest of the fits. The accepted mass and width of the $a_{2}$ is 1.318 and 0.107 GeV, respectively, from the Particle Data Group [2]. The fitted mass is close to the accepted value but the width is wider and this is expected from the effects of fitting a peak that is close to the width of the bins.

Fit type 2 is the result of coupling the intensity and phase of the $2^{+} 1$ into a $\chi^{2}$ fit. Appendix A expands on the details of the $\chi^{2}$ functional form. The resulting fit has narrower widths but fails to describe the phase shift past $1.45 \mathrm{GeV}$. The phase shift is fit for a constant phase minus the phase of the $2^{+} 1$. Fig. 4.17 shows that for a constant phase minus a pure $2^{+} 1$ wave, the phase will decrease by about 3 radians 
from 1.2 to $1.5 \mathrm{GeV}$. The phase shift of the data and fit overlap at the very edges of the error bars and diverge at masses higher than $1.5 \mathrm{GeV}$. The fit is shown in Fig. 4.18 and resulted in a mass and width of $1.32 \pm 0.01$ and $0.14 \pm 0.01 \mathrm{GeV}$.

Fit types 3 and 4 include the $1^{-} 1$ and $2^{+} 1$ but type 4 expands the amplitude of the $1^{-} 1$ from the form in equation 4.37 to be expressed as

$$
a_{\pi_{1}}=C_{r e} a_{J=1}^{B W}\left(m_{0}, \Gamma_{0}, m\right)+D_{\text {complex }} a_{J=1}^{B W}\left(m_{0}^{\prime}, \Gamma_{0}^{\prime}, m\right) .
$$

The complex normalization coefficient, $D$, exists to account for a possible constant phase difference between the two poles, referring to one partial wave state with two masses. The result of fit 3 , illustrated in Fig. 4.19, is the $a_{2}$ is found with a mass of $1.343 \pm 0.003$ and a width of $0.174 \pm 0.003 \mathrm{GeV}$. With the E852 mass and width values for the $\pi_{1}(1400)$ of 1.37 and $0.385 \mathrm{GeV}$, the $\pi_{1}$ amplitude was fit to have a mass and width of $1.39 \pm 0.23$ and $0.58 \pm 0.05 \mathrm{GeV}$. The results initially look promising but the result of the fit of the phase is poor. In the resonance spectra the acceptance decreasing from 0.8 to $0.15 \%$ in the last five bins in the fit (shown in Fig. 4.9), the shift in phase difference is possibly a product of poorly known fits due to a combination of low statistics in the data (9 to 38 events per bin) and rapidly changing acceptance. Table 4.3 summarizes the fit results.

The mass-dependent fits to find the mass and width of the $\pi_{1}$ state are inconclusive because the change in phase of the data between the $P$ and $D$ waves is too large to be described by the interference of the $\pi_{1}(1400)$ and $a_{2}(1320)$, where the expected phase difference would be 1 radian between the mass range of 1.2 and $1.4 \mathrm{GeV}$ as shown in Fig. 4.17. Including the second mass to the $\pi_{1}$ amplitude resulted in two fitted masses for the $\pi_{1}$ of $1.1 \pm 4.4 \mathrm{GeV}$ and $1.51 \pm 0.11 \mathrm{GeV}$. The accepted $\pi_{1}$ states are $\pi_{1}(1400)$ and $\pi_{1}(1600)$. The final fit did not find the $\pi_{1}(1400)$ mass but did come 


\begin{tabular}{|c|c|c|c|c|c|c|c|}
\hline type & mass $a_{2}$ & width $a_{2}$ & counts & mass $\pi_{1}$ & width $\pi_{1}$ & mass $\pi_{1}$ & width $\pi_{1}$ \\
\hline 1 & 1.32 & 0.154 & 2690 & & & & \\
& \pm 0.01 & \pm 0.011 & \pm 98 & & & & \\
\hline 2 & 1.32 & 0.14 & 2678 & & & & \\
& \pm 0.01 & \pm 0.01 & \pm 151 & & & & \\
\hline 3 & 1.343 & 0.174 & 3062 & 1.39 & 0.58 & & \\
& \pm 0.003 & \pm 0.003 & \pm 49 & \pm 0.23 & \pm 0.05 & & \\
\hline 4 & 1.33 & 0.128 & 2484 & 1.1 & 0.1 & 1.51 & 0.52 \\
& \pm 0.08 & \pm 0.022 & \pm 376 & \pm 4.4 & \pm 2.4 & \pm 0.11 & \pm 1.21 \\
\hline \hline
\end{tabular}

Table 4.3: Summary of the mass-dependent fits, all masses and widths are in units of $\mathrm{GeV}$.

within the width of the $\pi_{1}(1600)$. The result of this fit is not conclusive and shows the limit of the statistics available. 

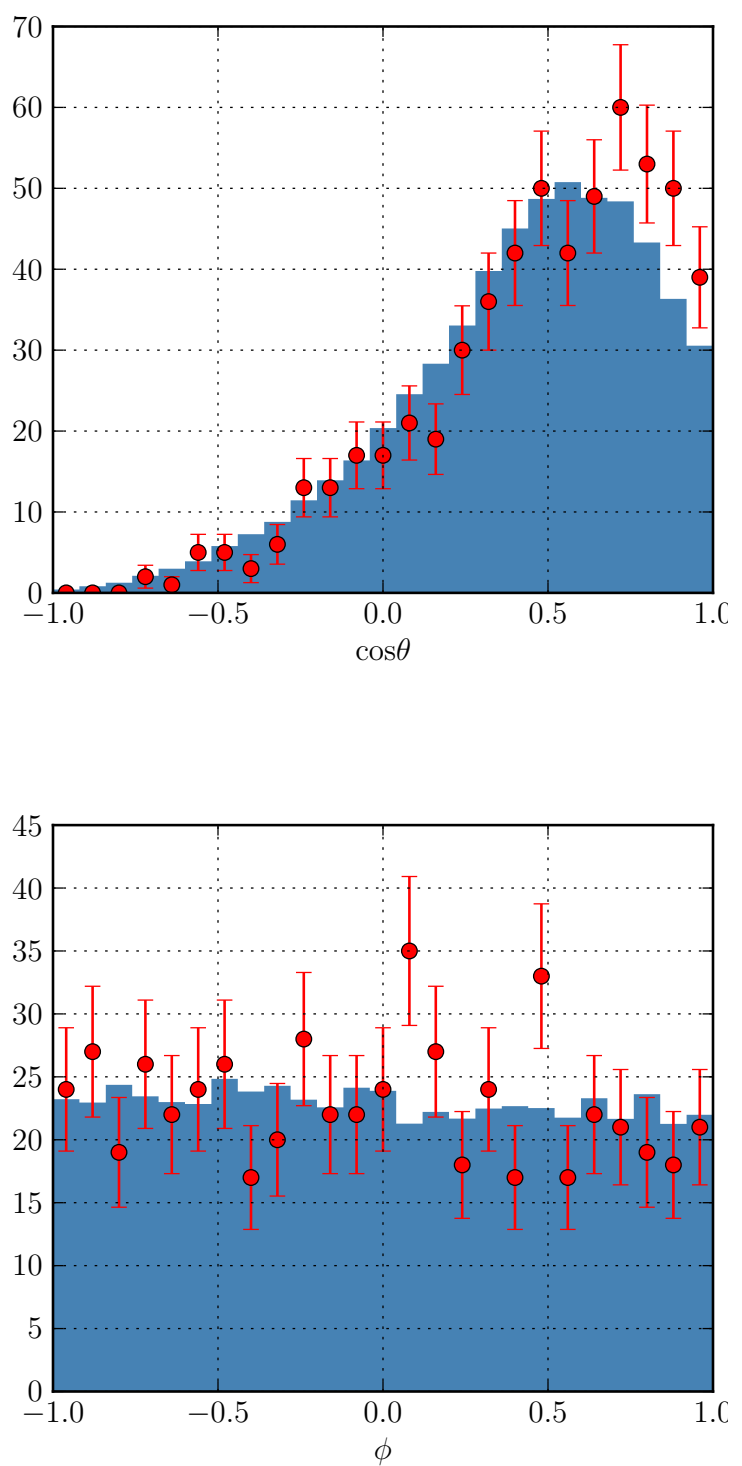

Figure 4.13: The GJ angles for the data (points) used in the PWA fit overlaid with the predicted distribution from the PWA solution (histogram). 

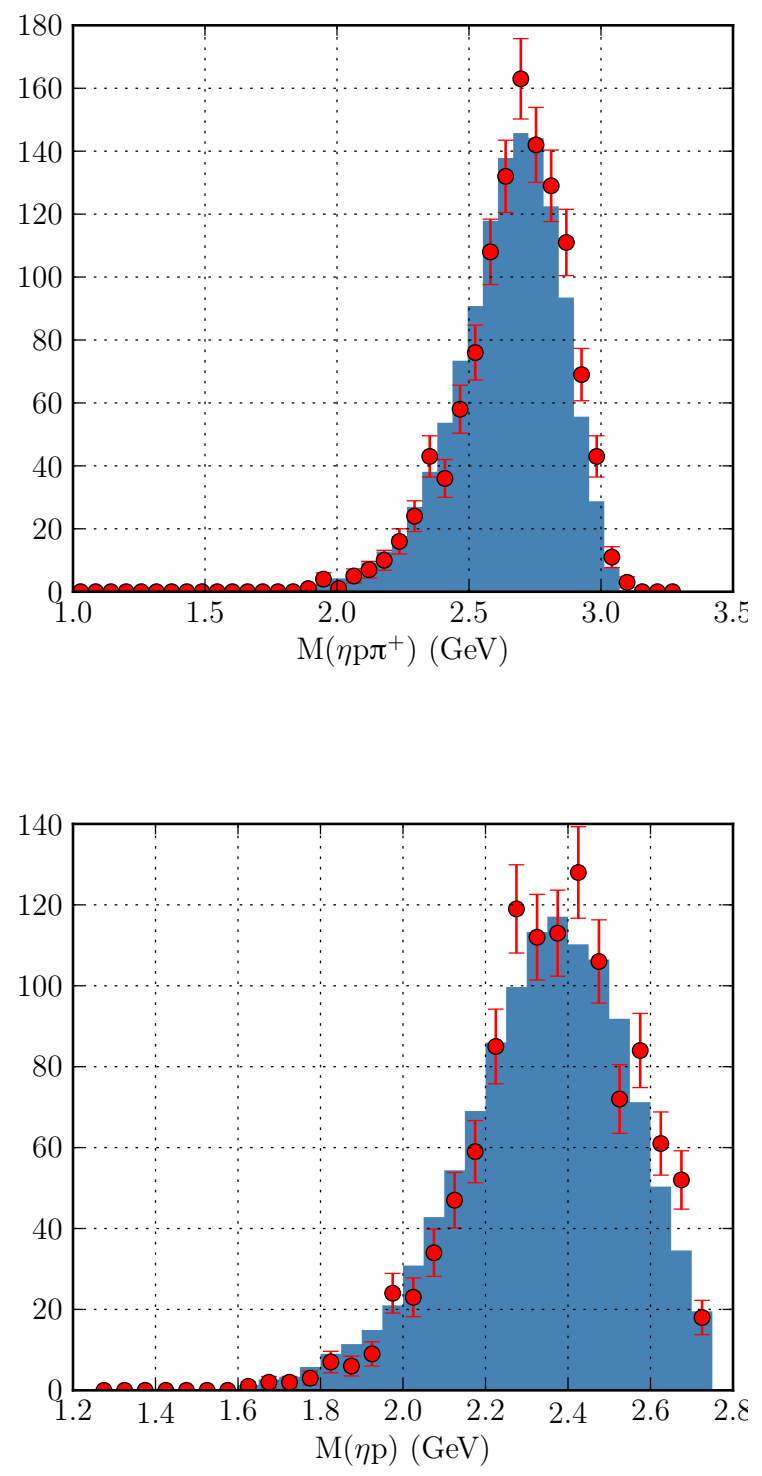

Figure 4.14: Invariant masses of $p \pi^{+} \eta$ and $p \eta$ of the data in red, with the invariant masses predicted by the PWA solution in blue. 

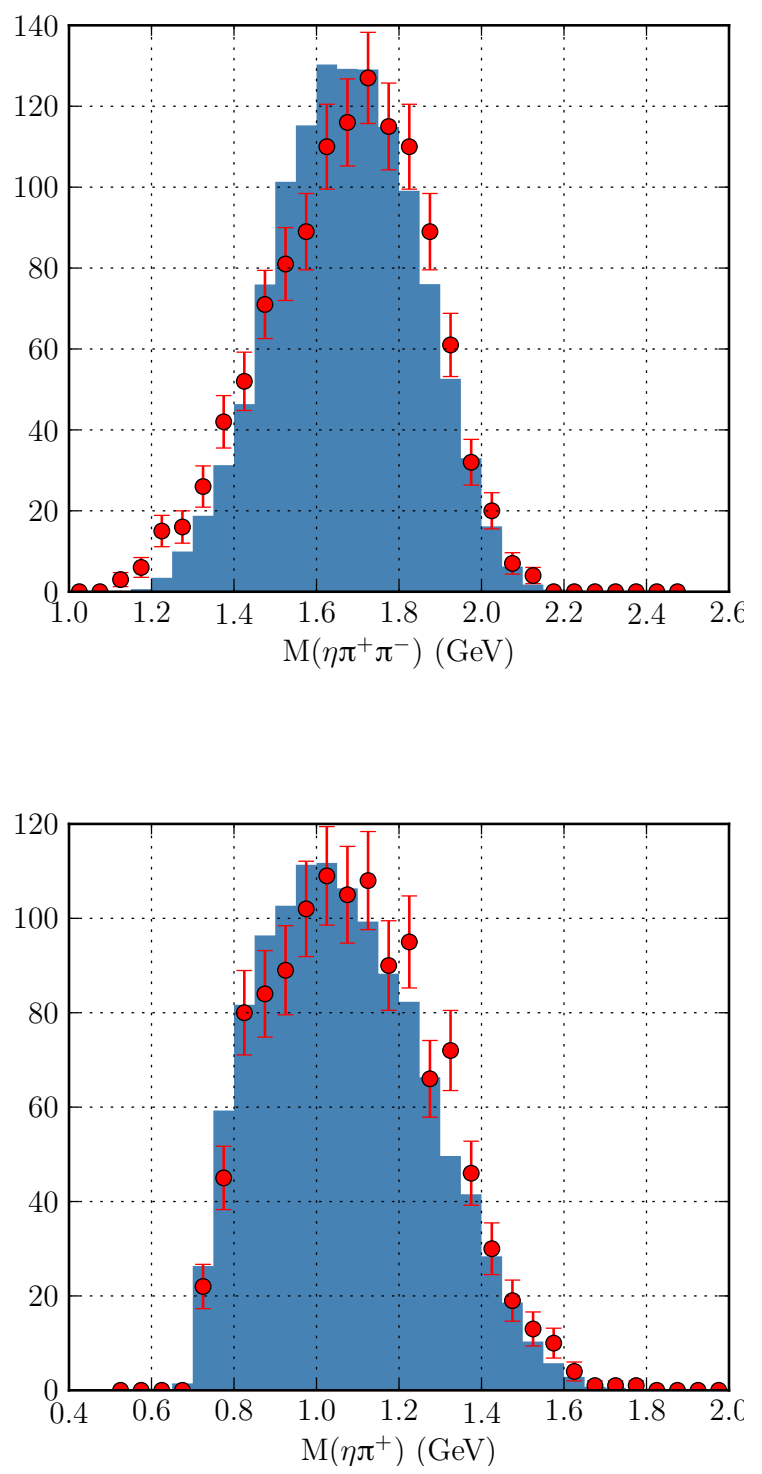

Figure 4.15: Invariant masses of $\pi^{+} \pi^{-} \eta$ and $\pi^{+} \eta$ of the data in red, with the invariant masses predicted by the PWA solution in blue. 


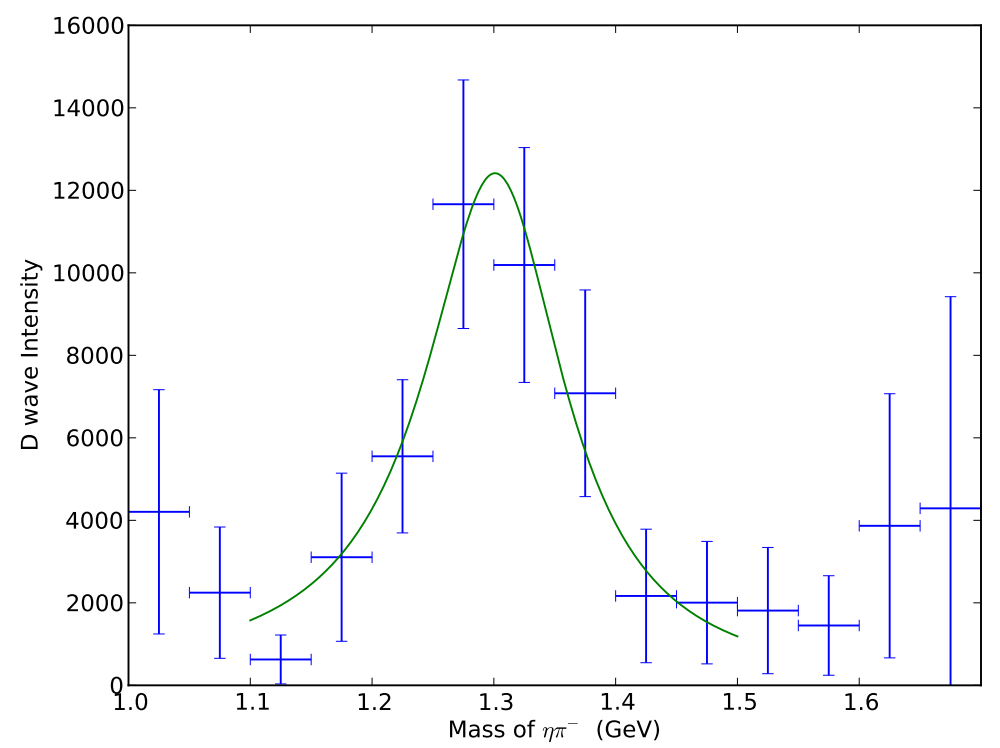

Figure 4.16: Fit 1: Intensity mass dependent fit for $a_{2}$. Fitting the BW intensity function (Eq. 4.36), the mass and width if the $a_{2}$ is $1.32 \pm 0.01$ and $0.154 \pm 0.011$ $\mathrm{GeV}$, includes both reflectivities added together. This included no contribution from the phase difference or the intensity of the $\pi_{1}$. 


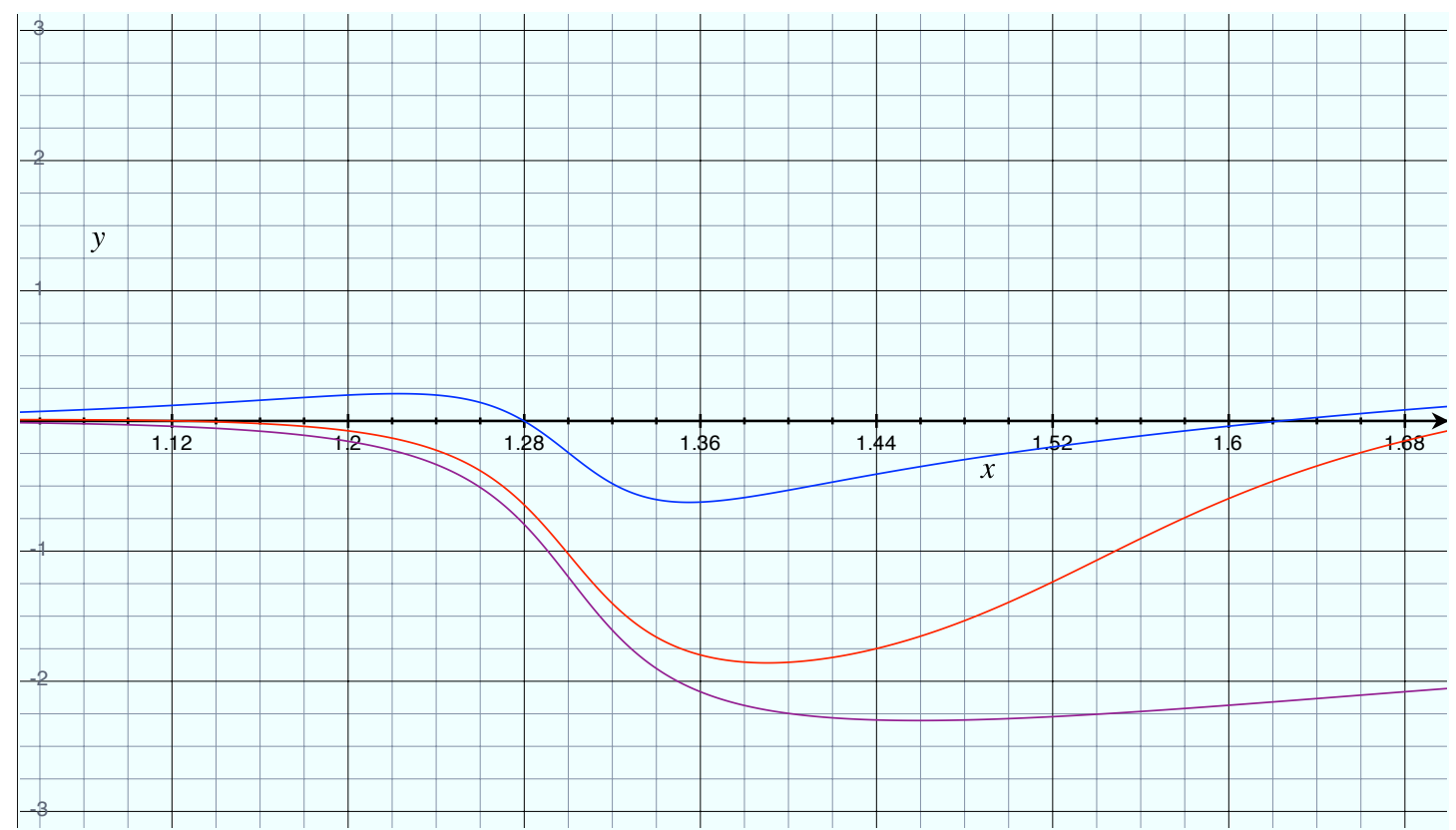

Figure 4.17: Calculated BW phase differences.

The $x$ axis is the resonance mass, the $y$ axis is the difference of $\phi$ calculated from equations 4.38 and 4.30 . The plot shows the expected phase for a $\pi_{1}(1400)$ minus $a_{2}(1320)$ in blue, $\pi_{1}(1600)$ minus $a_{2}(1320)$ in red, and constant phase minus $a_{2}(1320)$ in purple. 

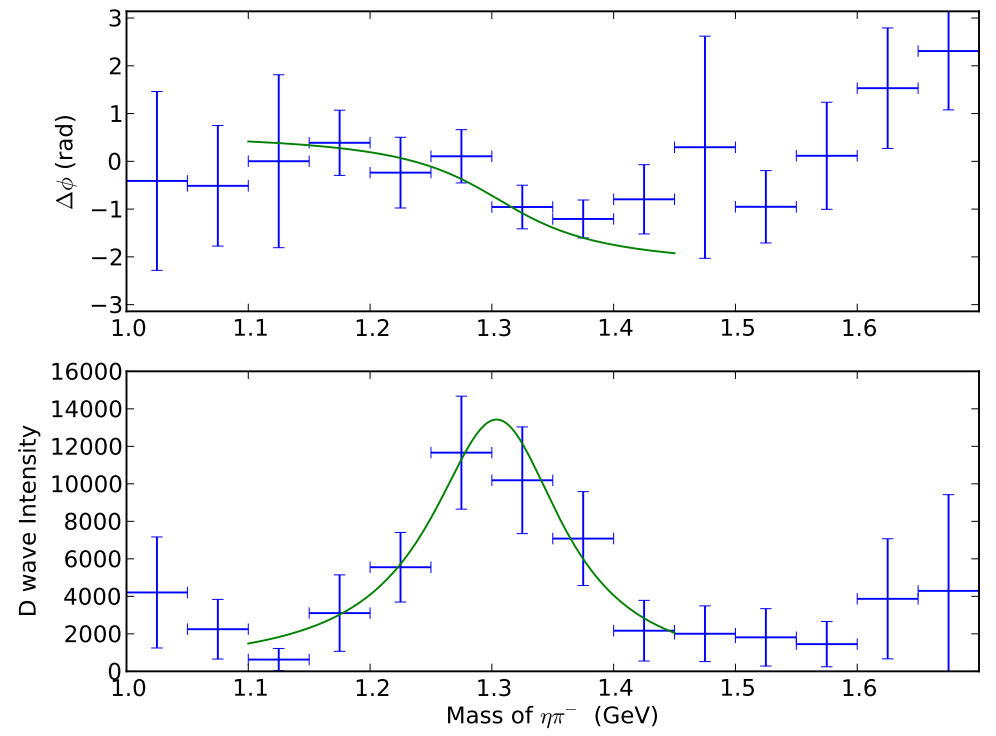

Figure 4.18: Fit 2: Coupled phase motion and intensity mass dependent fit for $a_{2}$. Fitting the BW intensity function, the mass and width if the $a_{2}$ is $1.32 \pm 0.01$ and $0.14 \pm 0.01 \mathrm{GeV}$, for positive reflectivity. The fit diverges at masses greater than 1.4 $\mathrm{GeV}$ in the phase difference. 


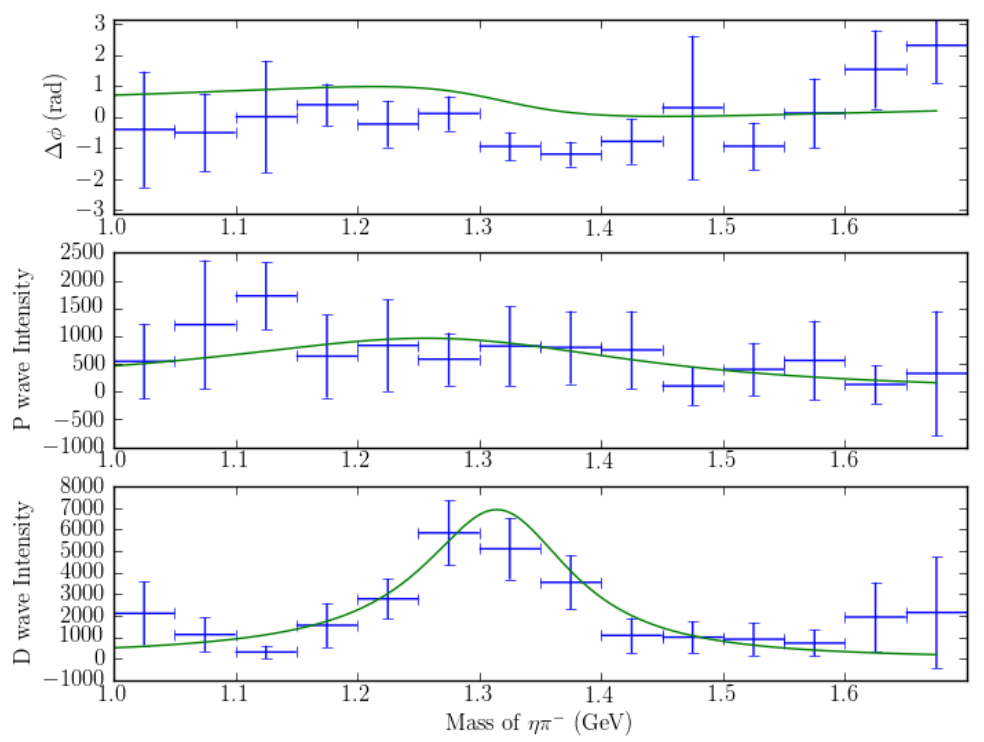

Figure 4.19: Fit 3: Coupled phase motion and intensity mass dependent fit for $a_{2}$ and $\pi_{1}$, for positive reflectivity. The mass and width of the fitted $a_{2}$ is $1.343 \pm 0.003$ and $0.174 \pm 0.003 \mathrm{GeV}$. The mass and width of $\pi_{1}$ is $1.39 \pm 0.23$ and $0.58 \pm 0.05 \mathrm{GeV}$ but the phase difference of the fit does not model the data. 


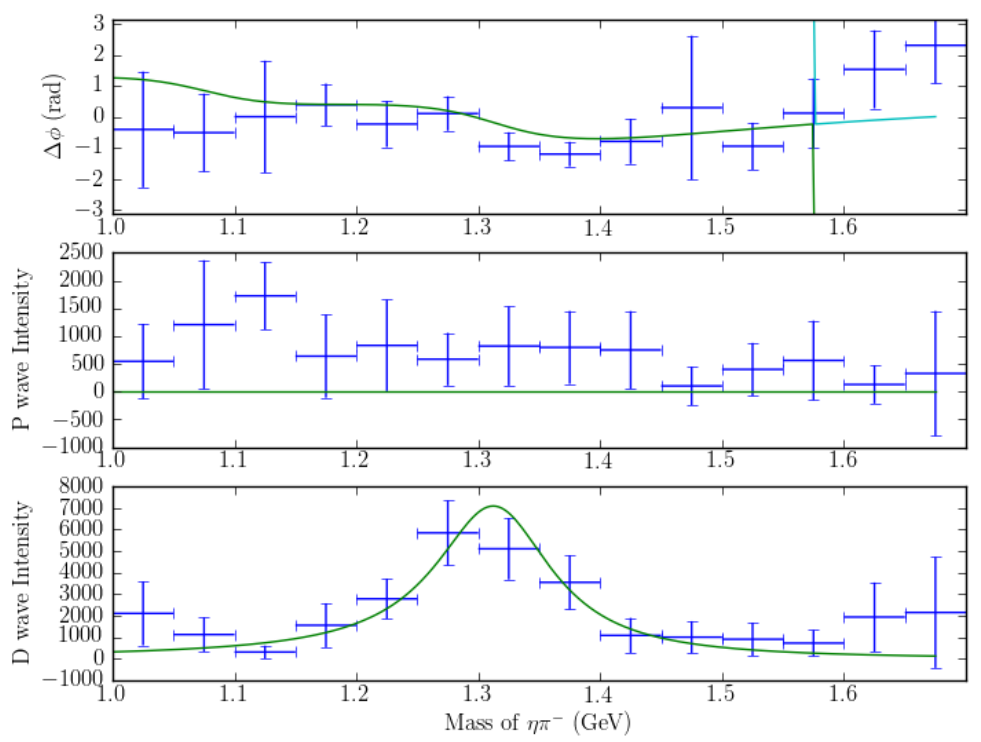

Figure 4.20: Fit 4: Coupled phase motion and intensity mass dependent fit for $a_{2}$ and $\pi_{1}$, for positive reflectivity. The mass and width of the fitted $a_{2}$ is $1.33 \pm 0.08$ and $0.128 \pm 0.022 \mathrm{GeV}$. The mass and width of $\pi_{1}$ is $1.1 \pm 4.4$ and $0.1 \pm 2.4 \mathrm{GeV}$ for the first pole and $1.51 \pm 0.11$ and $0.52 \pm 1.21 \mathrm{GeV}$ for the second. 


\subsection{Upper Limit on the $\pi_{1}(1400)$}

The cross section is a measure of the probability for two particles to interact. At the subatomic level a particle is replaced with a probability density and is no longer treated as a point particle. The cross section of a reconstructed particle is, $\sigma$ :

$$
\sigma=\frac{N}{L},
$$

where $L$ is the integrated luminosity over the energy range of the data used in the analysis and $N$ is the acceptance-corrected number of particles. The luminosity is calculated from the density and length of the target, as well as the number of incident photons. With $L$ a function of the experimental setup, the ratio of the cross section of $\pi_{1}$ to $a_{2}$ is

$$
\frac{\sigma_{\pi_{1}}}{\sigma_{a_{2}}}=\frac{N_{\pi_{1}}}{N_{a_{2}}},
$$

where $N_{\pi_{1}}$ and $N_{a_{2}}$ are the total number of counts for the $\pi_{1}$ and $a_{2}$ respectively.

This section presents the calculation of the upper limit estimation of the $\pi_{1}$ to $a_{2}$ cross section ratio. From the mass-dependent fit of the $a_{2}$ intensity, the yield is the integral of the mass dependent function fit to the $J^{P} m=2^{+} 1$ wave contribution from 0 to $\infty$. From various fits, there are approximately $54,580 a_{2}$ events with a systematic uncertainty of $\pm 4,900$, where the number of $a_{2}$ events is the integral of the Breit-Wigner form divided by the bin size. The fitted function of the intensity does not include a background function because any background under the $a_{2}$ peak in the initial $\eta \pi^{-}$mass is sorted out in the amplitude analysis.

The partial wave analysis of the $\eta \pi^{-}$shows no clear peak in the $1^{-} 1$ wave contributions. The data from this wave in the final fit are shown in Fig. 4.10(b). The $\pi_{1}(1400)$ expected mass and width taken from the E852 analysis is $1370 \pm 16 \mathrm{MeV}$ and 385 $\pm 40 \mathrm{MeV}$. Using the mass and width of the $\pi_{1}(1400)$ from E852 and setting them as fixed values for a mass-dependent fit, the yield from the integral from 0 to $\infty$ is 


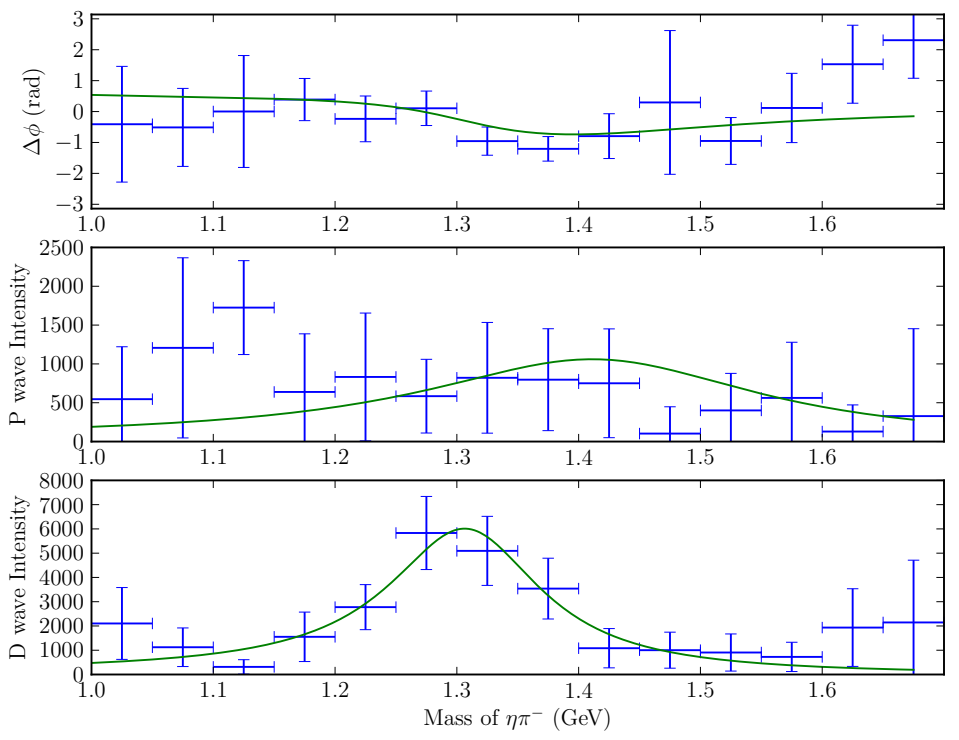

Figure 4.21: Coupled Phase Motion and Intensity Mass Dependent Fit for $a_{2}$ and $\pi_{1}$, using a fixed $\pi_{1}$ mass and width from E852 of $1370 \pm 16 \mathrm{MeV}$ and $385 \pm 40 \mathrm{MeV}$.

19,340 with a CL of $95.4 \%$ for the $\pi_{1}$. The upper limit of the cross section of $\pi_{1}(1400)$ is $\sigma\left(\gamma p \rightarrow \Delta^{++}\left(\pi_{1}^{-} \rightarrow \eta \pi^{-}\right)\right)=0.35$ (C.L. of $\left.95.4 \%\right) \times \sigma\left(\gamma p \rightarrow \Delta^{++}\left(a_{2}^{-} \rightarrow \eta \pi^{-}\right)\right)$. The uncertainty was calculated from the uncertainty in the fitted magnitude of the $\pi_{1}$ when the mass and width is fixed. The upper limit of the $\pi_{1}$ yield is calculated to be the fitted yield plus two $\sigma$.

Considering the bins covering the expected mass range of the $\pi_{1}$, the sum of the events is $14,686 \pm 4674 \pm 9008$ for the $1^{-}$. For the $a_{2}$, the sum of the events is 49,518 $\pm 7090 \pm 2652$. Where the statistical uncertainty is calculated by the sum of the squares of the error bars of the yields and the systematic uncertainty is calculated from the results of multiple PWA results. The upper limit of the cross section of $\pi_{1}(1400)$ is $\sigma\left(\gamma p \rightarrow \Delta^{++}\left(\pi_{1}^{-} \rightarrow \eta \pi^{-}\right)\right)=0.30( \pm 0.21) \times \sigma\left(\gamma p \rightarrow \Delta^{++}\left(a_{2}^{-} \rightarrow \eta \pi^{-}\right)\right)$. 


\section{CHAPTER 5}

\section{DISCUSSIONS AND CONCLUSIONS}

In summary, the reaction $\gamma p \rightarrow \Delta^{++} \eta \pi^{-}$was analyzed to search for a $J^{P C}=1^{-+}$ exotic meson state. The invariant mass of the $X$ shows a peak consistent with the presence of the non-exotic $a_{2}(1320)$. The PWA of the $\eta \pi^{-}$system shows partial wave contributions from the $J^{P}|m|=2^{+} 1$ state are consistent with the $a_{2}(1320)$. No statistically significant evidence was seen for partial waves from the $\pi_{1}(1400)$. The mass-dependent fits of the interference between the $J^{P}|m|=2^{+} 1$ and $1^{-} 1$ finds the $a_{2}$ but the $\pi_{1}$ results do no consistently reconstruct the $\pi_{1}(1400)$ when the second pole is added for the possibility of the $\pi_{1}(1600)$ and did not explain the phase shift.

This analysis started with the hypothesis of photoproduction will produce an exotic state comparable to the $a_{2}$ state, from Ref. [4]. The idea of choosing this analysis channel was the $\Delta^{++}$will filter out background contributions from other interfering channels. The expected spin projection $|m|$ is limited to 1 from the spin projection of the incident photon. Additional higher order waves were included but the contributions were negligible and made no difference in the outcome of the fit. For $1^{-+} 0$ and $2^{++} 0$ waves, the contributions turned out to be negligible. Including the $\Delta^{++}$simplified the wave set by restricting the available states.

The results show the upper limit cross section estimate is about $1 / 4$ th of the $a_{2}$ cross section, implying the $\pi_{1}$ is not produced in photoproduction comparable to the estimates theorized, which were 50 to $100 \%$ of the $a_{2}$. It is possible the upper limit found in this analysis contains $\pi_{1}$ events with a cross section comparable to the E852 results but to refine the upper limit to the actual cross section will require a PWA on the same mass range but with narrower mass bins and a larger data sample.

The goal of analysis was to find the partial wave combination that contribute to the final stated of $\eta \boldsymbol{\pi}^{-}$. The contributions from the $a_{2}(1320)$ were found. However, 
the exotic $\pi_{1}(1400)$ remains just as elusive and mysterious as when g12 was first proposed. Although the exotic wave is not as pronounced compared to the $a_{2}$ as thought to be in photoproduction and with the $\pi_{1}(1400) 5.5 \%$ of the $a_{2}$ intensity in E852, it would be beneficial to look at this channel again with higher statistics. 


\section{BIBLIOGRAPHY}

[1] N. Isgur and J. Paton. Flux-tube model for hadrons in qcd. Phys. Rev. D, 31:2910-2929, (1985).

[2] H. Bichsel. Passage of particles through matter, (2006).

[3] S. U. Chung. Evidence for exotic $j^{p c}=1^{-+}$meson production in the reaction $\pi^{-} p \rightarrow \eta \pi^{-} p$ at $18 \mathrm{gev} /$ c. Phys. Rev. D, 60, (1999).

[4] A. P. Szczepaniak. Role of photo production in exotic meson searches. Phys. Lett. B, 516:72-76, (2001).

[5] Povh. Particles and Nuclei: An Introduction to the Physical Concepts. Springer, 2 edition, (1999).

[6] D. Griffiths. Introduction to Elementary Particles. Wiley-VCH, (2004).

[7] F. Yndurain. The Theory of Quark and Gluon Interactions. Springer, (2006).

[8] M. Gell-Mann. A schematic model of baryons and mesons. Phys. Lett., 8(3):214215, (1964).

[9] G. Zweig. CERN preprint 8419/Th412, 8182/Th401.

[10] D. R. Thompson. Evidence for exotic meson production in the reaction $\pi^{-} p \rightarrow$ $\eta \pi^{-} p$ at $18 \mathrm{gev} / \mathrm{c}$. Phys. Rev. Lett., 79(9), (1997).

[11] C. Amsler. Mesons beyond the naive quark model. Physics Reports, 387:61-117, (2004).

[12] A. Abele. Evidence for a $\pi \eta$-wave, with odd angular momentum in anti-protonproton-annhilations at rest into $\pi^{0} \pi^{0} \eta$. Phys. Lett. B, 446(349), (1999).

[13] A. Abele. Exotic $\eta \pi$ state in antiproton-deuterium annihilation at rest into $\pi^{-} \pi^{0} \eta p_{\text {spectator. }}$ Phys. Lett. B, 423(175), (1998).

[14] D. S. Carman. Gluex: The search for gluonic excitations at jefferson laboratory. In Hadron Spectroscopy: Eleventh International Conference, (2006). 
[15] P. Eugenio. Search for new forms of hadronic matter in photoproduction. JLABE04-005, (2003).

[16] R. Feynman. Photon-hadron interactions. Westview Press, (1998).

[17] M. Clark. Non-diffractive photoproduction of vector meson. Nuclear Materials $B, 125: 493-512$, (1977).

[18] B. A. Mecking. The cebaf large acceptance spectrometer (clas). Nucl. Instr. and Meth. A, 503:513, (2003).

[19] Y. G. Sharabian. A new highly segmented start counter for the clas detector. Nucl. Instr. and Meth. A, 556(1):246-258, (2006).

[20] J. Ross. Development in the design of the superconducting toroidial magnet for the continuous electron beam accelerator facility (cebaf) large acceptance spectrometer. CLAS-NOTE, (92-015), (1992).

[21] M. Mestayer. The clas drift chamber system. Nucl. Instr. and Meth. A, 449:81111, (2000).

[22] Johann Goetz. $\Xi$ Hyperon Photoproduction from Threshold to $5.4 \mathrm{GeV}$ with the CEBAF Large Acceptance Spectrometer. PhD thesis, University of California, (2010).

[23] E. S. Smith. The time-of-flight system for clas. Nucl. Instr. and Meth. A, 432:265-298, (1999).

[24] M. Amarian. The clas forward electromagnetic calorimeter. Nucl. Instr. and Meth. A, 460(2-3):239-265, (2001).

[25] J. Li. The new tagger calibration program. CLAS-NOTE, (2003-004), (2003).

[26] E. Pasyuk. Energy loss corrections for charged particles in clas. CLAS-NOTE, (2007-016), (2007).

[27] D. Keller. Techniques in kinematic fitting. CLAS-NOTE, (2010-015), (2010).

[28] R. Mattuck. A Guide to Feynman Diagrams in the Many-Body Problem. Courier Dover Publications, (1976). 
[29] S. U. Chung. Formulas for partial-wave analysis version ii. BNL-QGS-93-05, (1993).

[30] D. J. Herndon. Generalized isobar model formalism. Phys. Rev. D, 11(11), (1975).

[31] J. H. Jee. The bnl four-body partial wave analysis system. based on BNL-QGS93-05.

[32] G. S. Adams. Exotic meson spectroscopy from bnl e852. In Hadron Spectroscopy: Eleventh International Conference, (2006).

[33] J. J. Manak. Partial-wave analysis of the $\eta \pi^{+} \pi^{-}$system produced in the reaction $\pi^{-} p \rightarrow \eta \pi^{+} \pi^{-} n$ at 18 gev/c. arXiv:hep-ex/0001051v1 21Jan2000, (2000).

[34] M. Nozar. Search for the photo-excitation of exotic mesons in the $\pi^{+} \pi^{+} \pi^{-}$ system. CLAS-ANALYSIS, (2006-102), (2006).

[35] S. Chung. Spin formalisms. BNL, (76975-2006-IR), (2006).

[36] M. Rose. Elementary Theory of Angular Momentum. Number 57-8893. Wiley, (1957).

[37] J. Cummings. An object-oriented approach to partial wave analysis. arXiv:physics/0309052v1.

[38] S. Chung. Positivity conditions on the spin density matrix: A simple parameterization. Phys. Rev. D, 11(633), (1975).

[39] g12 simulation wiki page, (2012).

[40] A. Bevan. Afit user guide, (2010).

[41] P. Bevington. Data Reduction and Error Analysis for the Physical Sciences. Mc Graw Hill, third edition, (2003).

[42] A. Ostrovidov. private communication: Pwa error propagation, (2011). 


\section{APPENDIX A}

\section{APPENDIX}

Results for wave set $2^{+} 0^{-}, 2^{+} 1,1^{-} 0^{-}, 1^{-} 1$ and $0^{+} 0^{-}$ 


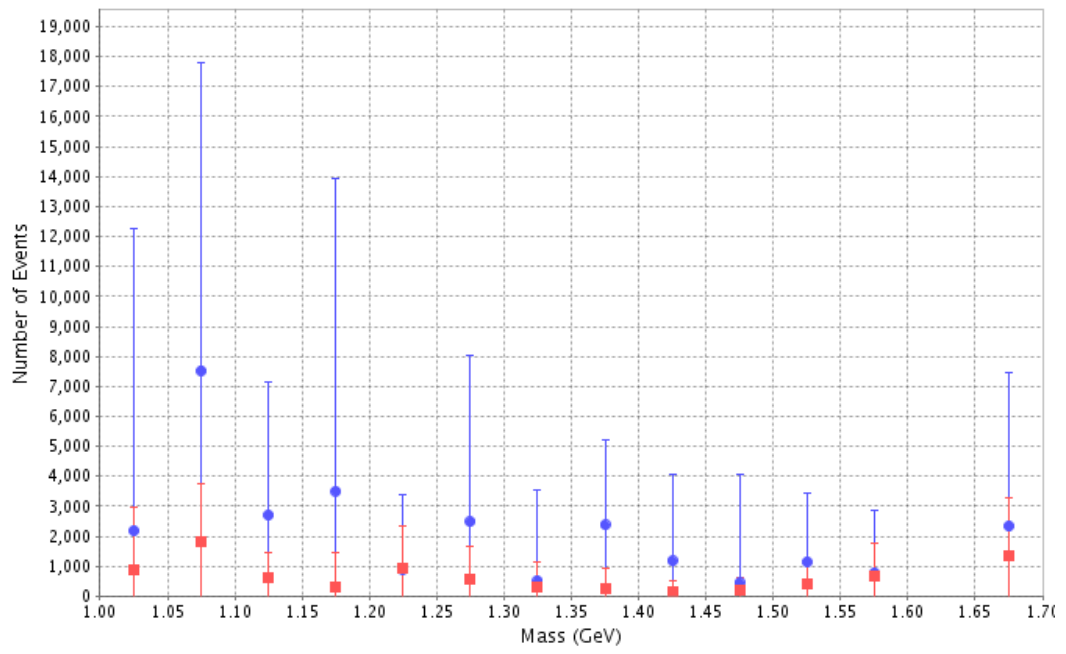

(a) Intensity of $2^{+} 0^{-}$(red) and $1^{-} 0^{-}$(blue)

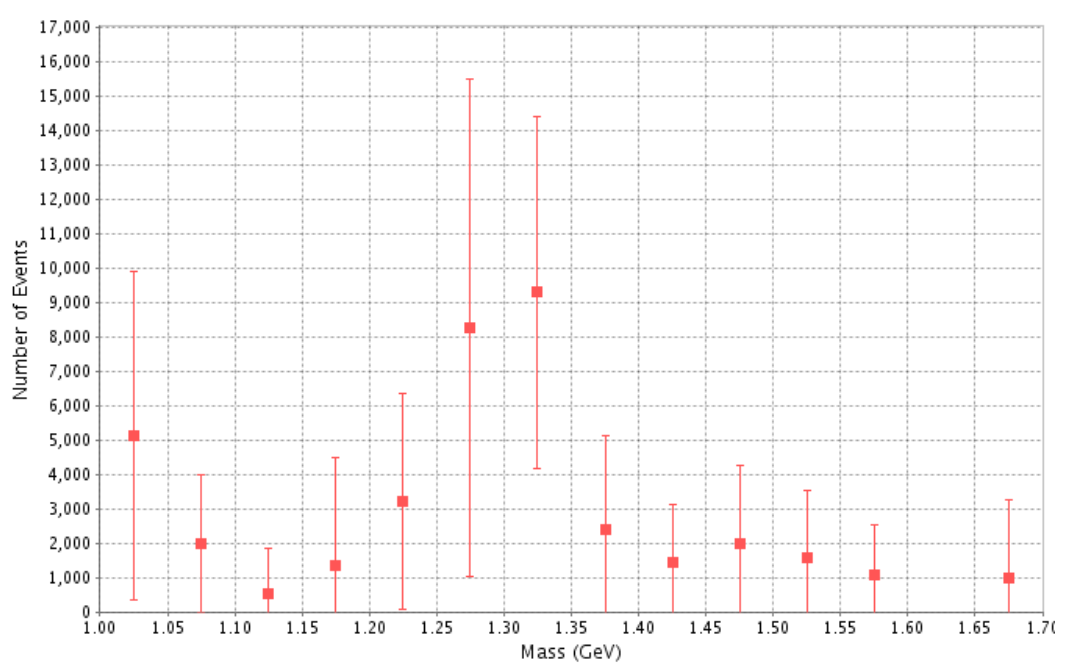

(b) Intensity of $2^{+} 1$

Figure A.1: Intensity distributions for the PWA using waves $2^{+} 0^{-}, 2^{+} 1,1^{-} 0^{-}, 1^{-} 1$ and $0^{+} 0^{-}$. 


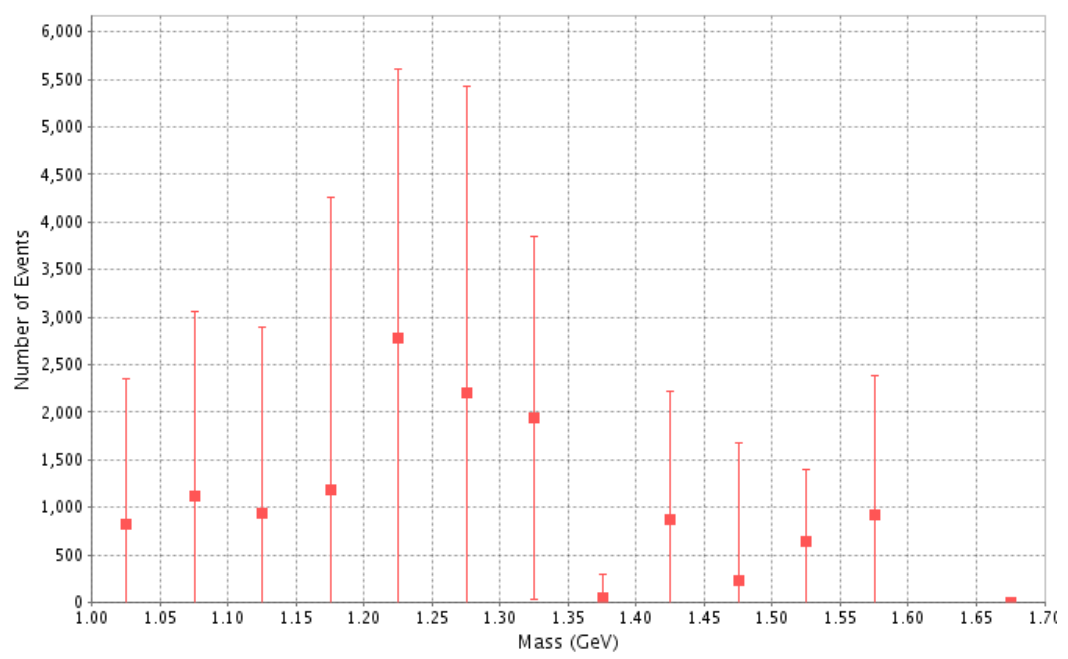

(a) Intensity of $1^{-1}$

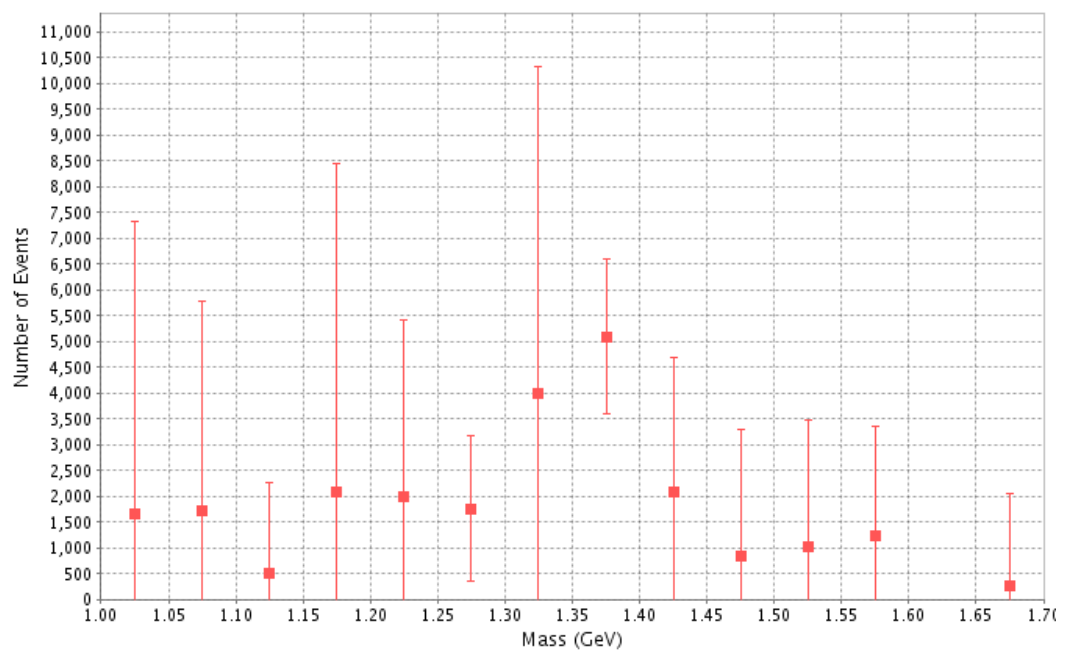

(b) Intensity of $0^{+} 0^{-}$

Figure A.2: Intensity distributions for the PWA using waves $2^{+} 0^{-}, 2^{+} 1,1^{-} 0^{-}, 1^{-} 1$ and $0^{+} 0^{-}$. 
Results for wave set $2^{+} 0^{-}, 2^{+} 1,1^{-} 1$ and $0^{+} 0^{-}$ 


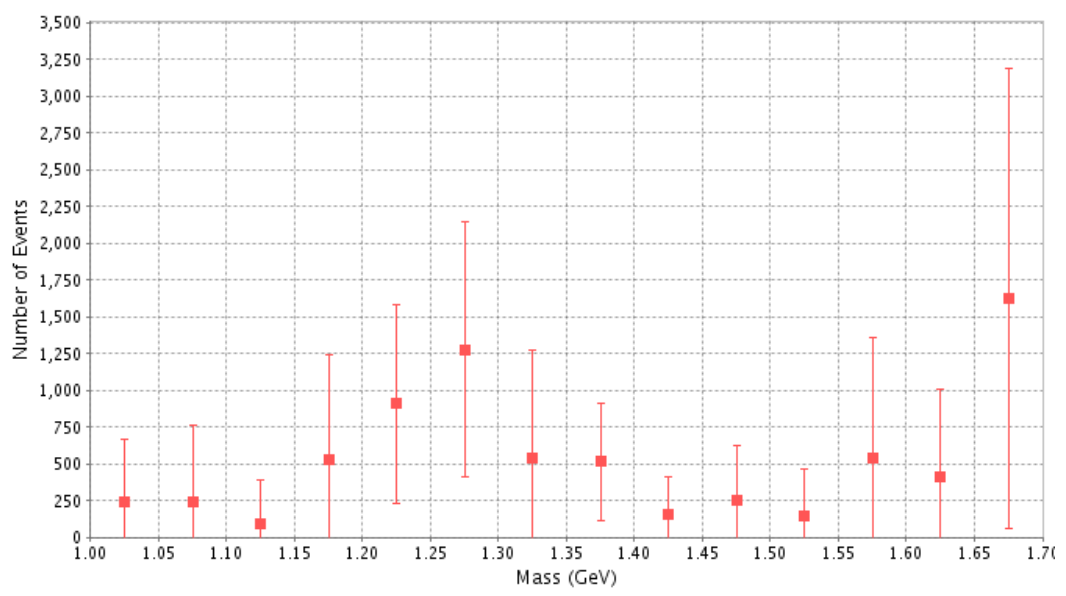

(a) Intensity of $2^{+} 0^{-}$

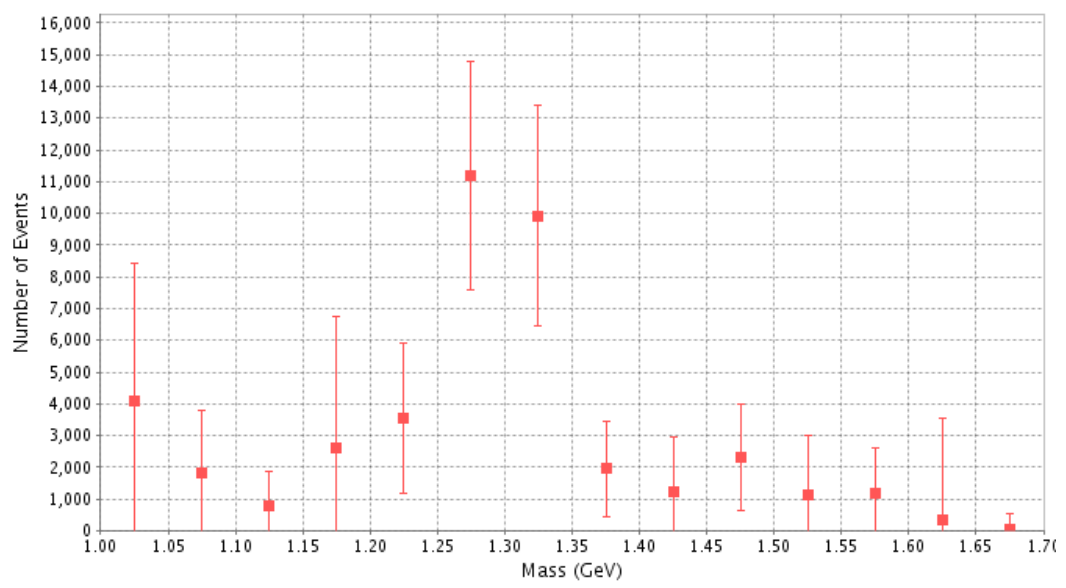

(b) Intensity of $2^{+} 1$

Figure A.3: Intensity distributions for the PWA using waves $2^{+} 0^{-}, 2^{+} 1,1^{-} 1$ and $0^{+} 0^{-}$. 


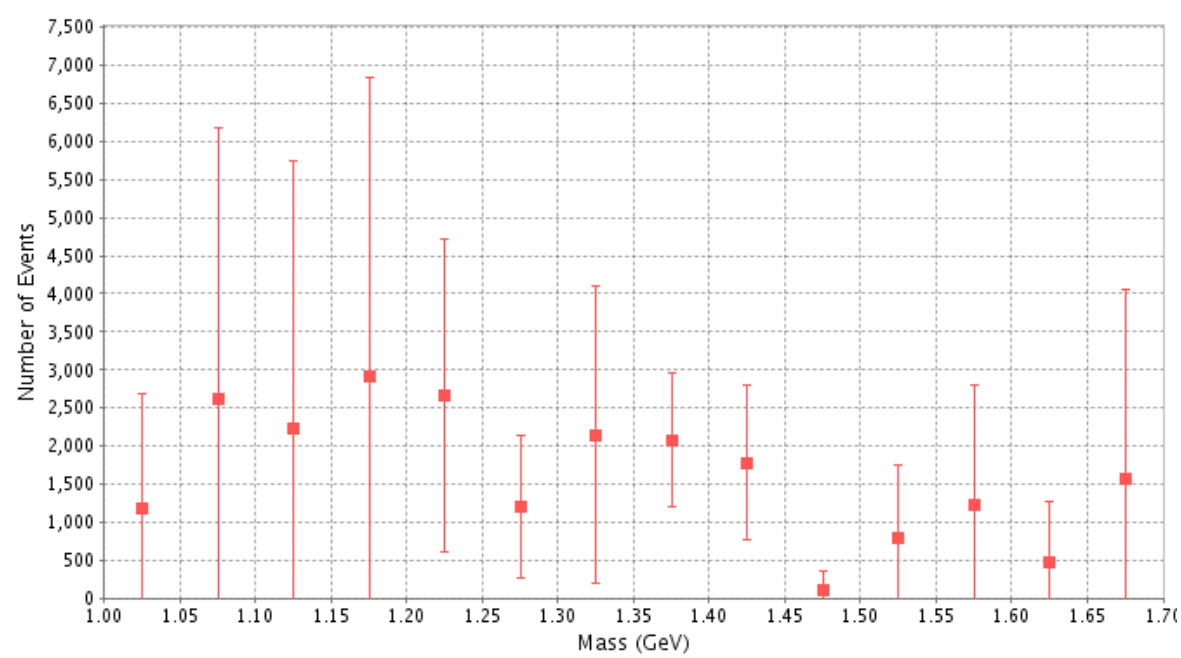

(a) Intensity of $1^{-1}$

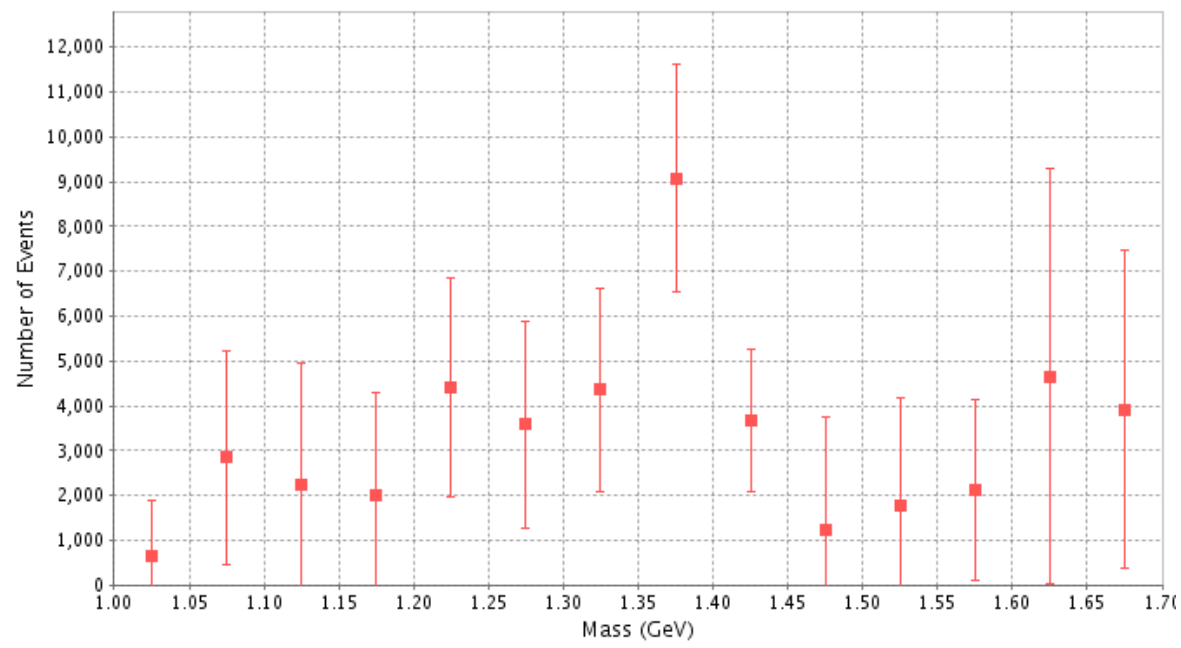

(b) Intensity of $0^{+} 0^{-}$

Figure A.4: Intensity distributions for the PWA using waves $2^{+} 0^{-}, 2^{+} 1,1^{-} 1$ and $0^{+} 0^{-}$. 


\section{Mass-Independent Fit Uncertainty}

To calculate the variance for $x$ as a function of $u$ and $v$, the error propagation equation [41] is defined as

$$
\sigma_{x}^{2} \simeq \sigma_{u}^{2}\left(\frac{\delta x}{\delta u}\right)^{2}+\sigma_{v}^{2}\left(\frac{\delta x}{\delta v}\right)^{2}+\ldots+2 \sigma_{u v}^{2}\left(\frac{\delta x}{\delta u}\right)\left(\frac{\delta x}{\delta v}\right)+\ldots
$$

It is used to calculate the uncertainties of the PWA final fit parameters into a final uncertainty for fit parameters that could possibly be correlated. For the PWA fit the results include the final production amplitudes, $V_{0}$ to $V_{w}$, and the covariance matrix calculated by the fitter, for $w$ number of waves. The covariance matrix is a $2 w$ by $2 w$ matrix, where the total number of waves included in the fit is $w$ but the production amplitudes are complex resulting in $2 w$ fit parameters. The uncertainty in the yield for a set of waves is calculated from the production amplitudes,

$$
V_{i}=a_{i}+i b_{i}
$$

and the raw normalization integral,

$$
R_{i j}=r_{x}^{i j}+i r_{y}^{i j}
$$

$V_{i}$ is a complex number for wave $i$ in the output of the PWA fitter described in section 4.4. $R_{i j}$ is the sum of $\rho_{\epsilon_{i} \epsilon_{j}} A_{i}^{*} A_{j}$ divided by the number of raw simulation events. The raw events are the generated events before the events are processed in the CLAS simulation. The final acceptance corrected yield of a set of waves is defined as

$$
\begin{aligned}
N & =\frac{n}{\eta} \sum_{i, j}^{w} V_{i}^{*} R_{i j} V_{j} \\
& =\frac{n}{\eta} M,
\end{aligned}
$$

where the sum is over $i, j$, for every combination of waves in the set. $n$ is the number of data events in the fitted bin and $\eta$ is the acceptance. 
The fitter outputs a covariance matrix to express the variance between every combination of $V_{i}$ elements. Using equation $\mathrm{A}$, the uncertainty in the yield of the set of waves is

$$
\sigma_{N}^{2} \simeq \frac{n}{\eta} \sum_{i, j}^{w}\left[\sigma_{a_{i}} \sigma_{a_{j}} \frac{\delta M}{\delta a_{i}} \frac{\delta M}{\delta a_{j}}+2 \sigma_{b_{i}} \sigma_{a_{j}} \frac{\delta M}{\delta b_{i}} \frac{\delta M}{\delta a_{j}}+\sigma_{b_{i}} \sigma_{b_{j}} \frac{\delta M}{\delta b_{i}} \frac{\delta M}{\delta b_{j}}\right] .
$$

The $\sigma_{u} \sigma_{v}$ are the $u$-th column and $v$-th row of the covariance matrix from the output of the fitter. The partial derivatives are calculated from:

for $i>j$

$$
\begin{aligned}
& \frac{\delta M}{\delta a_{i}}=2 R_{i i} a_{i}+2 \sum_{j}\left[r_{x}^{i j} a_{j}+r_{y}^{i j} b_{j}\right] \\
& \frac{\delta M}{\delta b_{i}}=2 R_{i i} b_{i}+2 \sum_{j}\left[r_{x}^{i j} b_{j}-r_{y}^{i j} a_{j}\right]
\end{aligned}
$$

and for $i<j$

$$
\begin{aligned}
& \frac{\delta M}{\delta a_{j}}=2 R_{j j} a_{j}+2 \sum_{i}\left[r_{x}^{i j} a_{i}-r_{y}^{i j} b_{i}\right] \\
& \frac{\delta M}{\delta b_{j}}=2 R_{j j} b_{j}+2 \sum_{i}\left[r_{x}^{i j} b_{i}+r_{y}^{i j} a_{i}\right] .
\end{aligned}
$$

The phase of a complex production amplitude, equation $\mathrm{A}$, is defined as

$$
\phi_{i}=\tan ^{-1} \frac{b_{i}}{a_{i}}
$$

and the phase phase difference between two waves can be expressed as

$$
\Delta \Phi_{1,2}=\phi_{2}-\phi_{1}
$$

To calculate the uncertainty in $\Delta \Phi_{1,2}$, the derivatives of $\Delta \Phi_{1,2}$ are calculated with respect to the output variables of the fitter $\left(a_{i}\right.$ and $\left.b_{i}\right)$. The derivatives are defined as:

$$
\frac{\delta \phi_{i}}{\delta a_{i}}=-\frac{b_{i}}{a_{i}^{2}+b_{i}^{2}}
$$

and

$$
\frac{\delta \phi_{i}}{\delta b_{i}}=\frac{a_{i}}{a_{i}^{2}+b_{i}^{2}}
$$


Following equation $\mathrm{A}$, the uncertainty of $\Delta \Phi$ is calculated from:

$$
\begin{aligned}
(\delta \Delta \Phi)^{2}= & \left(\frac{\delta \phi_{2}}{\delta b_{2}}\right)^{2}\left(\sigma_{b_{2}}\right)^{2}+\left(\frac{\delta \phi_{2}}{\delta a_{2}}\right)^{2}\left(\sigma_{a_{2}}\right)^{2}+\left(\frac{\delta \phi_{1}}{\delta b_{1}}\right)^{2}\left(\sigma_{b_{1}}\right)^{2}+\left(\frac{\delta \phi_{1}}{\delta a_{1}}\right)^{2}\left(\sigma_{a_{1}}\right)^{2} \\
& +2\left(\frac{\delta \phi_{1}}{\delta a_{1}}\right)\left(\frac{\delta \phi_{1}}{\delta b_{1}}\right)\left(\sigma_{a_{1}}\right)\left(\sigma_{b_{1}}\right)-2\left(\frac{\delta \phi_{1}}{\delta a_{1}}\right)\left(\frac{\delta \phi_{2}}{\delta a_{2}}\right)\left(\sigma_{a_{1}}\right)\left(\sigma_{a_{2}}\right) \\
& -2\left(\frac{\delta \phi_{1}}{\delta a_{1}}\right)\left(\frac{\delta \phi_{2}}{\delta b_{2}}\right)\left(\sigma_{a_{1}}\right)\left(\sigma_{b_{2}}\right)-2\left(\frac{\delta \phi_{1}}{\delta b_{1}}\right)\left(\frac{\delta \phi_{2}}{\delta a_{2}}\right)\left(\sigma_{b_{1}}\right)\left(\sigma_{a_{2}}\right) \\
& -2\left(\frac{\delta \phi_{1}}{\delta b_{1}}\right)\left(\frac{\delta \phi_{2}}{\delta b_{2}}\right)\left(\sigma_{b_{1}}\right)\left(\sigma_{b_{2}}\right)+2\left(\frac{\delta \phi_{2}}{\delta a_{2}}\right)\left(\frac{\delta \phi_{2}}{\delta b_{2}}\right)\left(\sigma_{a_{2}}\right)\left(\sigma_{b_{2}}\right)
\end{aligned}
$$

where $\left(\sigma_{u}\right)\left(\sigma_{v}\right)$ are the same matrix element of the covariance matrix as used in the yield calculation, and are the $(u, v)$ element of the matrix.

An alternative to directly calculating the uncertainty from the covariance matrix is to reiterate the PWA fit using random initial parameter values. To select the absolute minimum, the fit was reiterated ten times with randomly generated initial fitting parameters between -10.0 and 10.0, and the fit with the smallest $-\ln L$ was selected. The results of these various fits can be used to examine the uncertainty in the fit. For the mass bin $1250-1300 \mathrm{MeV}$, the mean of the fits are $1477 \pm 307$ for wave contributions is $P, 11787 \pm 120$ for $D$, and $4813 \pm 489$ for $S$. Compared to the values in Figs. 4.10 and 4.11, the uncertainty in the mean is much less than the uncertainty from the error propagation calculation, resulting in the mean and $\sigma_{\text {mean }}$ being used for monitoring purposes and not the final uncertainty calculation. The $\sigma_{\text {mean }}$ is calculated by

$$
\sigma^{2}=(\bar{N})^{2}-\left(\bar{N}^{2}\right)
$$

\section{Definition and Uncertainty Propagation of $\chi^{2}$ for Mass-Dependent Fit}

To combine the intensity and phase distribution into a $\chi^{2}$ function, the procedure used was adopted from the algorithm used by A. Ostrovidov in the E852 analysis [42]. The procedure translates the covariance matrix describing the variance of $V_{1}$, $V_{2}$, and $V_{1}^{*} V_{2}$ into the covariance matrix describing $N_{1}, N_{2}$, and $\Delta \Phi$, resulting in 
a final covariance matrix $E_{b} . \quad E_{b}$ is calculated from the following. Setting up the variables:

$$
A=\left(\begin{array}{llllll}
a_{1} & b_{1} & a_{2} & b_{2} & a_{12} & b_{12}
\end{array}\right)
$$

and

$$
B=\left(\begin{array}{llll}
N_{1} & N_{2} & a_{12} & b_{12}
\end{array}\right)
$$

Matrix $A$ is the set of initial parameters and matrix $B$ can be calculated from $A$. Using the covariance matrix $\sigma_{i} \sigma_{j}$ from the fit, a covariance matrix for the variables of matrix $A$ is assembled,

$$
E=\left(\begin{array}{cccccc}
\delta a_{1} \delta a_{1} & \delta b_{1} \delta a_{1} & \delta a_{2} \delta a_{1} & \delta b_{2} \delta a_{1} & \delta a_{12} \delta a_{1} & \delta b_{12} \delta a_{1} \\
\delta a_{1} \delta b_{1} & \delta b_{1} \delta b_{1} & \delta a_{2} \delta b_{1} & \delta b_{2} \delta b_{1} & \delta a_{12} \delta b_{1} & \delta b_{12} \delta b_{1} \\
\delta a_{1} \delta a_{2} & \delta b_{1} \delta a_{2} & \delta a_{2} \delta a_{2} & \delta b_{2} \delta a_{2} & \delta a_{12} \delta a_{2} & \delta b_{12} \delta a_{2} \\
\delta a_{1} \delta b_{2} & \delta b_{1} \delta b_{2} & \delta a_{2} \delta b_{2} & \delta b_{2} \delta b_{2} & \delta a_{12} \delta b_{2} & \delta b_{12} \delta b_{2} \\
\delta a_{1} \delta a_{12} & \delta b_{1} \delta a_{12} & \delta a_{2} \delta a_{12} & \delta b_{2} \delta a_{12} & \delta a_{12} \delta a_{12} & \delta b_{12} \delta a_{12} \\
\delta a_{1} \delta b_{12} & \delta b_{1} \delta b_{12} & \delta a_{2} \delta b_{12} & \delta b_{2} \delta b_{12} & \delta a_{12} \delta b_{12} & \delta b_{12} \delta b_{12}
\end{array}\right)
$$

where $\delta a_{12}=\sigma_{a_{1}} a_{2}+\sigma_{a_{2}} a_{1}+\sigma_{b_{1}} b_{2}+\sigma_{b_{2}} b_{1}$ and $\delta b_{12}=-\sigma_{a_{1}} b_{2}+\sigma_{a_{2}} b_{1}+\sigma_{b_{1}} a_{2}-\sigma_{b_{2}} a_{1}$. The next step is to calculate a matrix of derivatives of matrix $\mathrm{B}$ in terms of matrix A to form the matrix D:

$$
D=\left(\begin{array}{cccccc}
\frac{\delta N_{1}}{\delta a_{1}} & \frac{\delta N_{1}}{\delta b_{1}} & 0 & 0 & 0 & 0 \\
0 & 0 & \frac{\delta N_{2}}{\delta a_{2}} & \frac{\delta N_{2}}{\delta b_{2}} & 0 & 0 \\
\frac{\delta a_{12}}{\delta a_{1}} & \frac{\delta a_{12}}{\delta b_{1}} & \frac{\delta a_{12}}{\delta a_{2}} & \frac{\delta a_{12}}{\delta b_{2}} & 1 & \frac{\delta a_{12}}{\delta b_{12}} \\
\frac{\delta b_{12}}{\delta a_{1}} & \frac{\delta b_{12}}{\delta b_{1}} & \frac{\delta b_{12}}{\delta a_{2}} & \frac{\delta b_{12}}{\delta b_{2}} & \frac{\delta b_{12}}{\delta a_{12}} & 1
\end{array}\right)
$$

The new uncertainty matrix is now:

$$
E_{1}=D E D^{-1}
$$


and relates the initial uncertainty in the variables of matrix $A$ to matrix $B$. The next derivative matrix needed is the derivates of $N_{1}, N_{2}$, and $\Delta \Phi$ in terms of matrix $B$ :

$$
\begin{gathered}
D_{1}=\left(\begin{array}{cccc}
1 & 0 & 0 & 0 \\
0 & 1 & 0 & 0 \\
0 & 0 & \frac{\delta \Phi_{12}}{\delta a_{12}} & \frac{\delta \Phi_{12}}{\delta b_{12}}
\end{array}\right) \\
E_{2}=D_{1} E_{1} D_{1}^{-1}
\end{gathered}
$$

The final $\chi^{2}$ is defined as

$$
\begin{gathered}
\chi^{2}=\sum_{b=0}^{B} F_{b} E_{2, b}^{-1} F_{b}^{-1} \\
F_{b}=\left(\begin{array}{lll}
\Delta N_{1} & \Delta N_{2} & \Delta\left(\Delta \Phi_{1,2}\right)
\end{array}\right)
\end{gathered}
$$

where the sum is over the set of mass bins, $B$, and $F_{b}$ is a matrix of the difference between the predicted and the PWA calculated $N_{1}, N_{2}$, and $\Delta \Phi$. The predicted $N_{1}$, $N_{2}$, and $\Delta \Phi$ are calculated from equations 4.37 and 4.39 . The mass-dependent fitter varied the mass, width, and magnitude of the predicted values to minimize $\chi^{2}$.

The approximate uncertainties of the $\chi^{2}$ for parameters are calculated from the resulting covariance matrix of the mass-dependent fit. The uncertainty in a fit parameter is the square root of the corresponding covariance matrix element. 
VITA

\section{DIANE SCHOTT}

2005

2005-2007

2007-2012

2004-2012

2007-2012

2007-2012
B.S., Physics

Florida International University

Miami, Florida

Graduate Teaching Assistant

Florida International University

Miami, Florida

Graduate Research Assistant

Florida International University

Miami, Florida

Member of Users Group

Thomas Jefferson National Accelerator Facility

Newport News, Virginia

Member of CLAS Collaboration

Thomas Jefferson National Accelerator Facility

Newport News, Virginia

Member of American Physical Society

\section{PUBLICATIONS AND PRESENTATIONS}

D. Schott, "The Search for a $\pi_{1}(1400)$ Exotic Meson in the $\gamma p \rightarrow \Delta^{++} \eta \pi^{-}$System with CLAS," APS April 2012 Meeting

D. Schott, "The Search for a $\pi_{1}(1400)$ Exotic Meson in the $\gamma p \rightarrow \Delta^{++} \eta \pi^{-}$System with CLAS," Fall 2010 APS DNP

D. Schott, "The search for a $\pi_{1}(1400)$ in $\gamma p \rightarrow \Delta^{++} \eta \pi^{-}$System with CLAS," HADRON 2009, AIP Conf. Proc. 1257, 492-494 (2010). 